\title{
The Transcriptome Architecture of Polyomaviruses
}

3 Jason Nomburg ${ }^{1,2,3}$, Wei Zou ${ }^{4}$, Thomas C. Frost ${ }^{1,3}$, Chandreyee Datta ${ }^{5,6,7,8}$, Shobha

4 Vasudevan ${ }^{5,6,7,8}$, Gabriel J. Starrett ${ }^{9}$, Michael J. Imperiale $^{4,10}$, Matthew Meyerson ${ }^{1,2,11^{*}}$,

5 James A. DeCaprio ${ }^{1,3,12^{*}}$

$7 \quad{ }^{1}$ Department of Medical Oncology, Dana-Farber Cancer Institute, Boston MA

$8 \quad{ }^{2}$ Broad Institute of MIT and Harvard, Cambridge, MA

$9{ }^{3}$ Harvard Program in Virology, Harvard University Graduate School of Arts and Sciences, 10 Boston, MA

$11{ }^{4}$ Department of Microbiology and Immunology, University of Michigan, Ann Arbor,

12 Michigan

13 5Massachusetts General Hospital Cancer Center, Harvard Medical School, 185

14 Cambridge St, CPZN4202, Boston, MA

$15{ }^{6}$ Department of Medicine, Massachusetts General Hospital and Harvard Medical School, 16 Boston, MA

$17{ }^{7}$ Center for Regenerative Medicine, Massachusetts General Hospital, Harvard Medical

18 School, Boston, MA

19 BHarvard Stem Cell Institute, Harvard University, Cambridge, MA

$20{ }^{9}$ Laboratory of Cellular Oncology, CCR, NCI, NIH, Bethesda, MD, USA 
$21{ }^{10}$ Rogel Cancer Center, Ann Arbor, MI

$22{ }^{11}$ Department of Genetics, Harvard Medical School, Boston, MA

23 12Department of Medicine, Brigham and Women's Hospital, Harvard Medical School,

24 Boston, MA

25

$26{ }^{*}$ Correspondence to:

27 James A. DeCaprio

28 james_decaprio@dfci.harvard.edu

29

30 Matthew Meyerson

31 matthew_meyerson@dfci.harvard.edu 


\section{Abstract}

34 Polyomaviruses (PyV) are ubiquitous pathogens that can cause devastating human

35 diseases. Due to the small size of their genomes, PyV utilize complex patterns of RNA

36 splicing to maximize their coding capacity. Despite the importance of PyV to human

37 disease, their transcriptome architecture is poorly characterized. Here, we compare

38 short- and long-read RNA sequencing data from eight human and non-human PyV. We

39 provide a detailed transcriptome atlas for BK polyomavirus (BKPyV), an important

40 human pathogen, and the prototype PyV, simian virus 40 (SV40). We identify pervasive

41 wraparound transcription in PyV, wherein transcription runs through the polyA site and

42 circles the genome multiple times. Comparative analyses identify novel, conserved

43 transcripts that increase PyV coding capacity. One of these conserved transcripts

44 encodes superT, a T antigen containing two RB-binding LXCXE motifs. We find that

45 superT-encoding transcripts are abundant in PyV-associated human cancers. Together,

46 we show that comparative transcriptomic approaches can greatly expand known

47 transcript and coding capacity in one of the simplest and most well-studied viral families.

49 Introduction

50 Polyomaviruses (PyV) are ubiquitous pathogens that can cause devastating human

51 diseases (Jiang et al., 2009a) including polyomavirus-associated nephropathy (PVAN),

52 hemorrhagic cystitis, and bladder cancer associated with BKPyV (Starrett et al., 2021),

53 progressive multifocal leukoencephalopathy caused by JCPyV, Merkel cell carcinoma

54 caused by Merkel cell polyomavirus (MCPyV), and dermatosis caused by human 
55 polyomavirus 7 (HPyV7) (Jiang et al., 2009a; Nguyen et al., 2017). PyV have circular

56 double-stranded DNA genomes and express viral genes with distinct "early" and "late"

57 kinetics. Early and late transcripts are driven by a bi-directional central promoter, and

58 each terminate at their own polyA signal sequence located between the early and late

59 regions. The PyV early region encodes tumor or $\mathrm{T}$ antigens that promote cell cycle

60 progression and facilitate replication of the viral genome by host DNA polymerase. The

61 PyV late region originating from the common PyV promoter element on the opposite

62 genome strand encodes the structural proteins required for the generation of progeny

63 virions.

65 PyV transcripts undergo complex splicing to increase their coding capacity in the face of

66 their small $\sim 5 \mathrm{~kb}$ genomes. In addition to the major large T (LT) and small T (ST)

67 antigens, additional T antigen splice forms have been identified including transcripts that

68 generate truncated versions of LT (17kT, 57kT, truncT, and T' in SV40, MCPyV,

69 BKPyV, and JCPyV respectively), a "superT" antigen that contains a duplicated LxCxE

70 RB-binding motif in SV40, middle T (MT) in murine PyV (MPyV), and ALTO in MCPyV

71 (Abend et al., 2009; Carter et al., 2013; Freund et al., 1992; Kress et al., 1979; Shuda et

72 al., 2008; Smith et al., 1979; Trowbridge and Frisque, 1995; Zerrahn et al., 1993).

73 Although the diversity of late transcripts has been explored in SV40 (Good et al., 1988),

74 late transcript diversity in other PyV, including the major human pathogens, is poorly

75 characterized. To address this lack of knowledge of PyV transcription and to discover

76 unannotated biologically relevant PyV-encoded protein products, we used long- and 
77 short-read RNA sequencing technologies to characterize the transcriptomes of eight

78 human and non-human PyVs.

\section{RNA sequencing expands PyV transcript diversity.}

82 To expand known PyV transcript diversity, we conducted a series of viral infections

83 followed by total or polyA short-read Illumina RNA sequencing (short-RNAseq (total)

84 and short-RNAseq (polyA) respectively) (Figure 1A). We integrated this newly

85 generated data with publicly available data from infected cell culture, human skin, and

86 other settings (Table 1). Viruses studied include SV40, BKPyV Dunlop variant and Dik

87 (wild type, or archetype), JCPyV, MPyV, MCPyV, HPyV7, and bark scorpion

88 polyomavirus 1 (BSPyV1).

90 For SV40 and the BKPyV Dunlop variant, which replicate robustly in cell culture, we

91 complemented short-read sequencing with Nanopore direct RNA sequencing

92 (dRNAseq) and PacBio Single-Molecule Real-Time sequencing (SMRTseq) (Figure

93 1A), two long-read sequencing approaches for polyA RNA with distinct library

94 preparations and sequencing strategies. Resultant RNAseq reads from long- and short-

95 read sequencing strategies were mapped against the viral reference genome and

96 grouped into transcript classes based on the presence of shared introns as detailed in

97 the Methods (Figure 1B). For SV40, viral transcripts represented $11.6 \%$ and $8.8 \%$ of 
98 transcripts in dRNAseq and SMRTseq, respectively. For BKPyV Dunlop, viral transcripts

99 represented $28.6 \%$ and $27.8 \%$ of transcripts in dRNAseq and SMRTseq, respectively.

100 The total number of viral reads is detailed in Figure S1A. Transcripts within the same

101 class contain the same introns but may have distinct transcript start sites (TSSs) and

102 transcript end sites (TESs). For most transcriptomes, the majority of transcripts are

103 members of the first few transcript classes (Figure S1B). To filter out erroneous splice

104 sites, we required that all introns present in a dRNAseq or SMRTseq read must also be

105 supported by at least 5 splice junction-spanning reads in short-RNAseq (total) data.

106 Detailed information on this transcript class strategy is present in the Methods.

Comparison of read coverage from short-RNAseq (total), dRNAseq, and SMRTseq

109 revealed that dRNAseq and SMRTseq were relatively consistent with read coverage, generally reflected expected patterns of exon usage (Figure 1C). In contrast, the read

111 coverage of short-RNAseq (total) was less representative of expected viral exon usage 112 and may reflect noise due to the amplification of smaller RNA fragments (Figure 1C).

114 For SV40 and BKPyV Dunlop, a transcript class (consisting of transcripts with shared

115 introns) was considered a bona-fide viral transcript if it was at least $0.1 \%$ of late or early

116 transcripts in dRNAseq or SMRTseq data as described in the Methods. For SV40,

117 which has detailed splice annotations (Good et al., 1988), we found that dRNAseq and

118 SMRTseq data are largely consistent with existing annotations. However, we identified

119 five previously unannotated SV40 transcripts that were supported by both long-read 
120 sequencing approaches, plus one additional previously unannotated SV40 transcript

121 class supported by SMRTseq and short-RNAseq (total) (Figure 1D, Figure 2).

123 In contrast to SV40 and despite its clinical importance, BKPyV transcripts have been

124 poorly characterized. We identified a total of 23 transcripts, 21 of which are supported

125 by both dRNAseq and SMRTseq data and only six of which were previously identified

126 (Abend et al., 2009; Seif et al., 1979) (Figure 1E, Figure 3). While novel BKPyV late

127 transcripts are often analogous to the characterized wraparound and non-wraparound

128 transcripts previously identified in SV40, several additional and unexpected BKPyV

129 early transcripts were identified. For example, an atypically early TSS revealed a splice

130 donor that was used to generate transcript E3 (Figure S4). Early transcripts including

131 E6, E9, and E11 are conserved across numerous PyV and lead to formation of novel

132 ORFs - these are described in detail below.

134 We generated a comprehensive atlas of SV40 and BKPyV transcripts in Figures S3 -

135 S8. Watch plots display the structure of each identified transcript, and read pileups

136 show all transcripts identified in each transcript class. The relative abundance of each

137 transcript as well as exact splice coordinates and abundance information for each

138 identified transcript is provided in Supplementary Tables 1 and 2. Transcripts can also

139 be explored using an interactive Google Colab notebook

140 (https://colab.research.google.com/github/jnoms/SV40 transcriptome/blob/main/bin/cola

$141 \underline{b / P y V}$ exploratory.ipynb). A comprehensive analysis of all splice sites detected in short- 
142 read short-RNAseq (total) and short-RNAseq (polyA) in eight PyV studied is presented

\section{3 in Figure S9.}

145 To address the possibility that distinct transcript isoforms could be preferentially

146 translated, we performed polysome profiling of SV40-infected cells coupled with

147 dRNAseq of whole-cell and polysome-associated polyadenylated RNAs (Figure 1F).

148 The ribosome occupancy, determined as the ratio between a transcript's normalized 149 polysome abundance and its normalized whole-cell abundance, has a mean of slightly

150 above 1 for host transcripts (Figure S2D). We found $11.2 \%$ of reads in the whole-cell

151 fraction and $18.7 \%$ in the polysome fraction were viral, consistent with active translation

152 of viral transcripts. For late transcripts, the relative abundance in the whole-cell fraction

153 was tightly coupled to polysome relative abundance (Figure 1G), indicating limited

154 preferential translation of late transcripts. In contrast to late transcripts, we found that

155 the LT:ST ratio was 1.3:1 in the polysome fraction compared to a 3.4:1 ratio of LT:ST

156 transcripts in the whole-cell fraction, indicating preferential translation of ST during

157 infection.

159 Wraparound transcription is conserved across diverse PyV.

160 Long-read sequencing revealed the existence of many late transcripts that contain

161 multiple copies of a duplicated leader exon. Leader-leader splicing is due to

162 "wraparound transcription" of PyV transcripts that failed to terminate at the late

163 polyadenylation signal and continue to circle the genome repeatedly. PyV wrapround 
164 transcription has been described previously although the structure and diversity of these

165 RNA species is unknown (Adami et al., 1989; Garren et al., 2015; Luo and Carmichael,

166 1991; Reddy et al., 1978). We investigated these transcripts in dRNAseq data from

167 SV40 and BKPyV. To supplement these data, we also performed dRNAseq on MPyV-

168 infected cells. Wraparound transcription, defined by the presence of repetitive copies of

169 a shared leader sequence, was found in long-read sequencing for all three PyVs

170 (Figure 4A, B, C: note the presence of the leader-leader or repeated exon near the "11

171 o'clock" position in watch plots). In addition to this leader sequence repetition, there are

172 diverse forms of wraparound transcripts that contain various combinations of

173 subsequent introns and encode for distinct viral proteins (Figure 2, 3). While only 3.6\%

174 of SV40 transcripts originate from wraparound transcription, BKPyV and MPyV have

175 markedly higher rates at $25 \%$ and $41 \%$ respectively (Figure 4D).

177 Next, we inferred the presence of wraparound transcription in diverse PyV by identifying 178 short-RNAseq (total) reads that span the leader-leader junction (Figure 4E). Despite the

179 limited length of these short reads, leader-leader junctions can be accurately identified 180 within a single read through analysis of junction sites (Figure 4F). We found evidence of 181 wraparound transcription in all eight PyV investigated here. This includes HPyV7

182 RNAseq from infected human skin and RNAseq data from a scorpion containing the 183 highly divergent Bark scorpion polyomavirus 1 (BSPyV1), indicating that wraparound 184 transcription occurs in vivo and is widely conserved across PyV. 
Pervasive premature polyadenylation of early transcripts in SV40, BKPyV, and MPyV.

We found that many early transcripts in SV40 and BKPyV underwent alternative polyadenylation (APA) earlier than the canonical polyA site as indicated by premature transcript end positions near 3 o'clock in the watch plots (Figure S10A, B). Early transcript APA had been previously identified in MPyV, where there is a canonical polyA signal sequence (AATAAA) within the LT ORF (Kamen et al., 1980b; Norbury and Fried, 1987). Indeed, dRNAseq identified APA of early transcripts in MPyV-infected cells (Figure S10C, D). In contrast to MPyV, APA in SV40 and BKPyV may be driven by alternative polyA signal sequences to the 5 ' of the APA site (ATTAAA in SV40, AAGAAA or TATAAA in BKPyV). Assessment of the cumulative incidence of early transcript termination shows abrupt increases in transcript termination $\sim 1500 \mathrm{nt}$ upstream of the canonical polyA site in all three viruses (Figure S10D). This APA appears to be similarly abundant in LT and ST transcripts. We found that transcripts with APA still contain a full polyA tail that, while shorter than the polyA tails of transcripts that use the canonical polyA site, still tend to be longer than the polyA tails of host transcripts (Figure S10E, S10F, S10G, S2C). The polyA tail length of a spike-in control RNA with a known 30-adenine polyA tail was correctly estimated by dRNAseq (Figure S2C). We find that transcripts containing APA can associate with polysomes (Figure $\mathrm{S} 10 \mathrm{H}, \mathrm{I})$, indicating that these transcripts are translated. 
209 Next, we conducted a comparative analysis of PyV transcription from short-RNAseq

210 (total) data (Figure S9), with the hypothesis that data from diverse PyV could reveal

211 unannotated splice forms. This analysis led to the discovery of several unannotated but

212 conserved splicing events that have the potential to expand the coding capacity of PyV

213 (Figure 5).

214

215 We found that PyVs including HPyV7, MPyV, BKPyV Dunlop, and MCPyV express a

216 transcript utilizing the LT first exon donor but an acceptor within the ST ORF leading to

217 the generation of the ST2 ORF (Figure 5A). This splice occurs in-frame in HPyV7 and

218 BKPyV resulting in an internal deletion within ST, while in MPyV and MCPyV this splice

219 lands out of frame and results in the addition of novel C-terminal amino acids. The ST2

220 splice is highly abundant in HPyV7 representing over $20 \%$ of spliced early transcripts

221 from HPyV7-infected human skin. ST2-encoding transcripts were detected in BKPyV

222 dRNAseq and SMRTseq data (transcript E6).

224 MPyV encodes MT in addition to the LT and ST antigens common with other PyV.

225 MPyV MT is generated from a splicing event that connects the ST ORF with an ORF in

226 the alternative frame of the LT second exon. To our surprise, we found that BKPyV

227 expresses low levels of a similar transcript containing a splice that connects the ST

228 ORF with an MT-like ORF likewise in an alternative frame of the LT second exon

229 (Figure 5B). This MT transcript was also detected in BKPyV dRNAseq and SMRTseq

230 data (transcript E9). 
232 JCPyV encodes two VP1 variants, VP1Xs, that consist of the N-terminal region of VP1

233 with novel C-termini that make up as much as $30 \%$ of late spliced transcripts in JCPyV

234 (Figure 5C) and have been recently identified and validated by an independent group

235 (Saribas et al., 2018). We found that VP1X-encoding transcripts were also produced by 236 MCPyV, SV40, BKPyV, and MPyV, albeit at a lower abundance than in JCPyV. Except

237 for one JCPyV VP1X-encoding splice, these transcripts were generated from splicing of 238 wraparound transcripts that run through the late polyA signal sequence.

SuperT, a T antigen containing two RB-binding motifs, is present in multiple PyV

241 and in PyV-associated human cancers.

242 Studies in SV40-transformed cells previously identified a superT antigen with higher

243 molecular weight than LT, containing a duplicated region with two copies of the LxCxE

244 RB-binding motif (Eul and Patzel, 2013). We found that a superT-specific splice was

245 present in SV40, BKPyV (Dik and Dunlop variants), JCPyV, and MCPyV during viral

246 infection (Figure 5D). The superT-specific splice originates from a splice donor

247 canonically associated with a conserved truncated LT antigen (17kT in SV40, truncT in

248 BKPyV, 57kT in MCPyV, and T' in JCPyV), but uses the LT second exon acceptor

249 available due to wraparound transcription. We find evidence of superT in the dRNAseq

250 and SMRTseq data for SV40 and BKPyV Dunlop infections (transcripts E4 and E11

251 respectively). Western blot with an antibody reactive to LT in BKPyV Dik-infected cells

252 revealed a band with slightly higher molecular weight than LT that is consistent with 
253 superT (Figure 5F). BKPyV Dik mutant M1, designed to remove ST by replacing the LT

254 intron with an intron from the plasmid $\mathrm{pCl}$ (Figure 5E), also generated a superT band of

255 expected size. BKPyV Dik mutant M2 was generated by removing the LT intron and

256 adding the $\mathrm{pCl}$ intron just 5' of the LT first exon. Should the truncT donor be used to

257 generate superT in this mutant, the only available acceptor is before the LT $1^{\text {st }}$ exon,

258 which would result in the formation of an aberrantly larger superT due to the inclusion of

259 a second copy of the LT first exon (Figure 5E). short-RNAseq (polyA) analysis of cells

260 infected with BKPyV Dik WT, M1, or M2 show junctions consistent with this model

261 (Figure S11), and western blot revealed that the superT band in M2 is shifted to a

262 higher molecular weight (Figure 5F). Together, these data indicate that superT is

263 generated by BKPyV Dik during viral infection.

SuperT was initially identified as an unexplained higher-molecular weight $\mathrm{T}$ antigen

266 present in many SV40-transformed cell lines (Kress et al., 1979; Smith et al., 1979).

267 While superT can be generated during viral infection because of wraparound

268 transcription, in SV40-transformed cells it would be possible to yield pre-mRNAs that

269 can be spliced to form superT should the virus be integrated in tandem copies (Figure

270 6A). Indeed, we previously observed that MCPyV integration events in Merkel cell

271 carcinoma (MCC) often lead to partial duplications of the viral genome and result in the

272 tandem insertion of multiple copies of viral early genes (Starrett et al., 2020).

273 Furthermore, the duplicated region in superT includes the RB-binding LxCxE motif,

274 raising the possibility that superT can function as a potent oncogene. We therefore

275 asked if there is evidence of superT in PyV-associated human cancers. 
277 To address this question, we first analyzed short-RNAseq (total) data from five BKPyV-

278 associated bladder cancers (Starrett et al., 2021). To our surprise, we found that short-

279 RNAseq (total) data from two replicates of one BKPyV-associated bladder cancer

280 contained a higher abundance of superT-specific splice than even the LT- or ST-specific

281 splices, suggesting that a large fraction of "LT" in this tumor is superT (Figure 6B). We

282 next analyzed short-RNAseq (polyA) data from a series of 30 MCPyV-positive MCCs

283 and found evidence of superT in six cases (Figure 6B). Notably, the total number of

284 viral reads in some MCPyV-positive but superT-null tumors was very low, leaving open

285 the possibility that sequencing depth was insufficient to identify the superT splice in 286 additional tumors. Using PCR and sanger sequencing, we confirmed the presence of

287 the superT splice in MCC tumor J45_440 (Figure S12A).

289 We hypothesized that superT may be generated by cis-splicing due to concatemeric 290 integration of multiple copies of the etiologic PyV in these tumors (Figure 6A). To

291 address this hypothesis, we investigated three MCCs (J45_440, J17_296, J11_285) for

292 which we possess short-read whole genome sequencing data. From J11_285, we were

293 able to assemble the entire integration site, showing that MCPyV is integrated in a

294 manner that could allow cis-splicing to generate superT (Figure S12B). For J45_440,

295 we assembled a single viral block integrated in chromosome 7 (Figure S12C). We

296 found that 1) there are likely 2 copies of the viral genome, and 2) the 5' viral integration

297 site appears to fall on chromosome 7 "after" the 3' viral integration site, observations

298 consistent with the existence of two copies of the viral genome in tandem separated by 
299

300

301

302

303

304

305

306

307

308

309

310

311

312

313

314

315

316

317

318

319

320

321

a small segment of host DNA at this integration site. For J17_296, from the assembly, we could infer three distinct segments of viral DNA with integration sites closely spaced within chromosome 2 (Figure S12D), indicating a complex integration pattern. The longer block contains two copies of the early region and can likely support superT generation through cis-splicing. The LT ORF of MCPyV is often truncated by premature stop codons or deletions in MCC. We found that a stop codon in J17_296 likely prevents expression of superT, but no stop codons occur before the superT splice in J45_440 or J11_285 (Figure S12E). Together, these data indicate that viral integration sites often could support cis-splicing to generate superT.

Two recent studies have found evidence of circular RNAs (circRNAs) that may be generated by MCPyV in MCC and may support the translation of ALTO (Abere et al., 2020; Yang et al., 2021). Of note, the major circRNA splice is equivalent to our proposed MCPyV superT splice - a short-RNAseq read spanning the proposed circRNA junction cannot be differentiated from a read spanning the superT junction. However, the MCC RNAseq samples in which we found superT are short-RNAseq (polyA), which should select against the potential circRNA due to its lack of a polyA tail. Furthermore, we detect the superT splice in short-RNAseq (polyA) of SV40 and BKPyV Dunlop infections in cell culture (Figure S9), although at around $\sim 2 / 3$ of its relative abundance in short-RNAseq (total). Finally, we identify full-length superT transcripts in dRNAseq data, which is highly unlikely to sequence circRNA since it is not polyadenylated. This leaves open the possibility that some superT-like splice in short-RNAseq (total) from viral infection originates from circRNA but suggests that most are from linear transcripts that contain a polyA tail. 


\section{Discussion}

324 Here, we show that leveraging multiple long- and short-read RNA sequencing

325 approaches across 8 polyomaviruses has allowed us to greatly expand known transcript

326 diversity of this viral family. Short read RNAseq has limited capacity to characterize

327 transcriptome diversity because only a small fraction of reads span splice junctions, and

328 these junctions often cannot be phased with other junctions or to the transcript start and

329 end sites. Integrating long-read sequencing has allowed sequencing of entire

330 transcripts, including phasing of splice sites and transcript start and end positions.

331 Recent studies have leveraged long read sequencing to shed light on exceptional

332 complexity in the transcriptomes of diverse RNA and DNA viruses (Balázs et al., 2017;

333 Depledge et al., 2019; Garalde et al., 2018; Keller et al., 2018; Kim et al., 2020;

334 Nomburg et al., 2020; Price et al., 2020). We have expanded these studies to show that

335 a comparative approach within a viral family can identify conserved transcripts that

336 extend viral coding capacity.

338 Historically, studies of PyV transcripts were limited by the sensitivity and resolution of

339 northern blots, or by the read length of short read sequencing. Despite these limitations,

340 studies in the 70's and 80's were able to cumulatively characterize several SV40 late

341 transcripts, including one containing leader-leader splicing (Ghosh et al., 1978; Good et

342 al., 1988; Reddy et al., 1978). In contrast to SV40, the architecture of BKPyV late

343 transcripts is poorly characterized - prior to this work, the two major classes of late 
344 transcripts ("16S" and "19S", reflecting transcript size based on gradient sedimentation

345 properties) were the primary late transcript classifications (Seif et al., 1979). Only

346 recently did a study provide some evidence for leader-leader splicing in BKPyV (Zou et

347 al., 2020). While the late transcripts of most PyV are thought to encode the canonical

348 late viral proteins, a recent study in JCPyV identified two splice events that lead to the

349 generation of novel proteins containing the N-terminal region of VP1 - one of which was

350 validated through western blot (Saribas et al., 2018). We found that these transcripts

351 (deemed "VP1X") are highly expressed in JCPyV but are also expressed at lower level

352 in BKPyV, MPyV, MCPyV, and SV40.

354 Leader-leader splicing is known to be highly prevalent in MPyV, where as many as 12 355 leader exons have been observed on a single RNA (Kamen et al., 1980a; Legon et al., 356 1979; Treisman, 1980) - in our data, we have identified over 15 leader exons in a single 357 transcript. Furthermore, leader-leader splicing is required for stable accumulation of 358 MPyV late transcripts, dependent on length but not nucleotide composition of the leader 359 (Adami et al., 1989). Despite these observations, the exact structure, diversity, and 360 conservation of wraparound transcripts was not understood. Here, we found that leader361 leader splicing and wraparound transcription occurs in all PyV studied, including in the 362 divergent Bark scorpion polyomavirus 1, and found that the prevalence of leader-leader 363 splicing varies significantly between PyV. It is possible that this variation reflects

364 differences in the strength of the late polyA signals of these PyV. We found a large 365 diversity of wraparound transcripts containing variable numbers of the leader sequence 366 and diverse patterns of subsequent exon usage. 
While late and early transcripts are thought to primarily end at the canonical late or early polyadenylation sites, studies previously observed APA of early transcripts in MPyV

370 (Kamen et al., 1980b; Norbury and Fried, 1987). Here, we likewise identify pervasive

371 APA of early SV40 and BKPyV Dunlop transcripts and find that SV40 early transcripts

372 with APA can associate with polysomes and are likely translated. In addition, polysome

373 profiling revealed that SV40 transcripts are higher abundance in polysome-associated

374 RNAs than in whole-cell RNA populations, indicating preferential translation of SV40

375 transcripts. The relative abundance of individual late viral transcripts in the polysome

376 closely reflected their whole-cell abundance - conversely, ST transcripts were

377 preferentially translated compared to LT transcripts. The mechanism driving this

378 difference needs further study, as these transcripts differ only by a minor difference in

379 splice donor usage.

381 In addition to the major early transcripts encoding LT and ST, other early transcripts

382 have been identified in some PyV. MPyV encodes MT, generated by a splice

383 connecting the ST ORF and an ORF overprinted with LT second exon. MT is a primary

384 oncogene in MPyV and was thought to be largely restricted to rodent PyVs (Gottlieb and

385 Villarreal, 2001). We found that BKPyV generates a MT-like ORF through splicing

386 connecting ST and an ORF similarly overprinted with the LT second exon, showing that

387 non-rodent PyVs may be capable of expressing MT-like ORFs. In addition, MPyV also

388 encodes a tinyT antigen consisting largely of the LT first exon, resulting from a splice

389 connecting the LT first exon donor and MT acceptor (Riley et al., 1997). We identified a 
novel T antigen, ST2, that is generated from a splice from the LT first exon donor to a

391 splice acceptor within the ST ORF. This transcript is highly expressed in HPyV7 and

392 present at lower levels in BKPyV, MPyV, and MCPyV. Many PyV encode a truncated

393 variant of LT - this includes SV40 17kT, BKPyV truncT, MCPyV 57kT and JCPyV T'

394 proteins (Abend et al., 2009; Shuda et al., 2008; Trowbridge and Frisque, 1995; Zerrahn

395 et al., 1993). These transcripts contain a canonical LT splice and a subsequent splice

396 that removes a large portion of the LT ORF.

We found that the same secondary splice sites responsible for truncated LT variants

can be used to generate superT. superT was initially observed in many SV40-

transformed cell lines (Kress et al., 1979; Smith et al., 1979) - in a similar manner, we

401 find that concatemeric integration of BKPyV and MCPyV in human cancers can facilitate

402 the generation of superT. We also find that superT is generated in lytic infections of

403 SV40, BKPyV, MCPyV and JCPyV. Eul and colleagues have published several studies

404 proposing that SV40 superT can be generated by trans-splicing between two separate

405 pre-mRNAs in the context of artificial expression constructs encoding the SV40 early

406 region $(20,31,32)$. However, we find that in MCC tumors that generate superT and for

407 which we can assemble the viral integration site, the viral genome is likely integrated in

408 tandem in a way that could facilitate the cis-splicing of pre-mRNA that spans multiple

409 genome copies. Thus, while we cannot rule out trans-splicing from these data, we

410 believe cis-splicing is more likely. Future studies are necessary to understand the

411 biology of superT including its oncogenic potential and ability to bind multiple RB

412 molecules. Finally, efforts should be taken to understand if superT is expressed by PyV 
413 and contributes to disease in other contexts, such as by BKPyV in PVAN or JCPyV in

414 PML.

415

416 We show that complex, uncharacterized splicing events are used by PyV to expand

417 their protein coding capacity. Future work is necessary to understand the biological

418 function of these transcripts and proteins. It is possible that unannotated splicing we

419 identify here could be differentially abundant in other biological contexts, so it will be

420 important to investigate PyV splicing in other infection contexts and human diseases.

421 Future transcriptome analyses that integrate long and short reads from multiple viruses

422 may have utility to expand characterized transcript and coding capacity in other viral

423 families.

425 Conclusions

426 We provide a comprehensive transcriptome atlas for the prototype PyV SV40, as well

427 as the critically important human pathogen BKPyV. Comparative analyses of PyV

428 transcriptomes reveals conserved splice events that may expand PyV coding capacity.

429 We find that superT, a transcript generated by SV40, BKPyV, JCPyV, and MCPyV that

430 encodes a T antigen containing two RB-binding LxCxE domains, is present in several

431 PyV-associated human cancers. Together, these data expand our understanding of PyV

432 transcriptomes and uncover unannotated PyV-encoded proteins of potential relevance

433 to human disease. 


\section{Materials and Methods}

\section{Data and code availability.}

438 All code used in this project can be found at the zenodo and github links below. The

439 zenodo repository also contains all processed data necessary to reproduce all analyses

440 and figures. The main processing steps used to process RNAseq data are present as

441 nextflow pipelines which call modular bash and python scripts.

442 Zenodo: https://doi.org/10.5281/zenodo.5593468

443 Github: https://github.com/jnoms/SV40 transcriptome

445 Furthermore, a series of interactive Google Colab notebooks can download all

446 processed data from Zenodo and completely reproduce all analyses and non-schematic

447 primary figures. The colab documents are stored on github at

448 https://github.com/jnoms/SV40 transcriptome/tree/main/bin/colab. Direct links to the

449 Google Colab documents are as follows:

450 Figure 1:

451 https://colab.research.google.com/github/jnoms/SV40 transcriptome/blob/main/bin/cola

452 b/Figure1.ipynb 
453 Figure 4:

454 https://colab.research.google.com/github/jnoms/SV40 transcriptome/blob/main/bin/cola

455 b/Figure4.ipynb

456 Figure 6:

457 https://colab.research.google.com/github/jnoms/SV40 transcriptome/blob/main/bin/cola

458 b/Figure6.ipynb

459

460 A Google Colab notebook is available for interactive investigation of all SV40 and

461 BKPyV viral transcript classes, and does not require computational skills to use:

462 https://colab.research.google.com/github/jnoms/SV40 transcriptome/blob/main/bin/cola

463 b/PyV exploratory.ipynb

464

465 All raw RNA sequencing data are available at the NCBI sequence read archive at

466 accession $\mathrm{XXXXXX.}$

467

468 Datasets

469 Information on all samples and viruses (excluding tumors) can be found in Table 1.

\begin{tabular}{|c|c|c|c|c|}
\hline Table 1 & Origin & MOI / Timepoint & Host \\
\hline Virus & Sequencing & & \\
& Type & (Accession) & & \\
\hline
\end{tabular}




\begin{tabular}{|c|c|c|c|c|}
\hline SV40 & $\begin{array}{l}\text { dRNAseq (two } \\
\text { replicates) }\end{array}$ & Generated here & MOI 1 / 48hpi & C. Sabaeus \\
\hline SV40 & SMRTseq & Generated here & MOI 1 / 48hpi & C. Sabaeus \\
\hline $\begin{array}{l}\text { SV40 (polysome } \\
\text { input/whole-cell) }\end{array}$ & dRNAseq & Generated here & MOI 1 / 44hpi & C. Sabaeus \\
\hline SV40 (polysome) & dRNAseq & Generated here & MOI 1 / 44hpi & C. Sabaeus \\
\hline SV40 & $\begin{array}{l}\text { Short-RNAseq } \\
\text { (total) }\end{array}$ & Generated here & MOI $1 / 48 \mathrm{hpi}$ & C. Sabaeus \\
\hline SV40 & $\begin{array}{l}\text { short-RNAseq } \\
\text { (polyA) }\end{array}$ & Generated here & MOI 1/48hpi & C. Sabaeus \\
\hline BKPyV (Dunlop) & dRNAseq & Generated here & MOI 0.5 / 3dpi & Human \\
\hline BKPyV (Dunlop) & SMRTseq & Generated here & MOI 0.5 / 3dpi & Human \\
\hline BKPyV (Dunlop) & $\begin{array}{l}\text { Short-RNAseq } \\
\text { (total) }\end{array}$ & Generated here & MOI 0.5 / 3dpi & Human \\
\hline BKPyV (Dunlop) & $\begin{array}{l}\text { short-RNAseq } \\
\text { (polyA) }\end{array}$ & Generated here & MOI 0.5 / 3dpi & Human \\
\hline BKPyV (Dik) WT & $\begin{array}{l}\text { Short-RNAseq } \\
\text { (total) }\end{array}$ & Generated here & MOI 1 / 5dpi & Human \\
\hline BKPyV (Dik) WT & $\begin{array}{l}\text { Short-RNAseq } \\
\text { (polyA) }\end{array}$ & Generated here & MOI 1 / 5dpi & Human \\
\hline BKPyV (Dik) M1 & $\begin{array}{l}\text { Short-RNAseq } \\
\text { (polyA) }\end{array}$ & Generated here & MOI 1 / 5dpi & Human \\
\hline
\end{tabular}




\begin{tabular}{|c|c|c|c|c|}
\hline BKPyV (Dik) M2 & $\begin{array}{l}\text { Short-RNAseq } \\
\text { (polyA) }\end{array}$ & Generated here & MOI 1 / 5dpi & Human \\
\hline MPyV & dRNAseq & Generated here & Unknown / 28hpi & Mouse \\
\hline MPyV & $\begin{array}{l}\text { Short-RNAseq } \\
\text { (total) }\end{array}$ & $\begin{array}{l}\text { Garren et al. (Garren et al., } \\
\text { 2015) (SRR2043214) }\end{array}$ & MOI 50 / 36hpi & Mouse \\
\hline JCPyV & $\begin{array}{l}\text { Short-RNAseq } \\
\text { (total) }\end{array}$ & $\begin{array}{l}\text { Assetta et al. (Assetta et al., } \\
\text { 2016) (SRR9967610) }\end{array}$ & Unknown / 9dpi & Human \\
\hline $\begin{array}{l}\text { MCPyV (Synthetic } \\
\text { genome) }\end{array}$ & $\begin{array}{l}\text { short-RNAseq } \\
\text { (polyA) }\end{array}$ & $\begin{array}{l}\text { Theiss et al. (Theiss et al., 2015) } \\
\text { (EBI: ERS760222) }\end{array}$ & $\begin{array}{l}\text { 200ng viral DNA / } \\
\text { Unknown }\end{array}$ & Human \\
\hline HPyV7 & $\begin{array}{l}\text { Short-RNAseq } \\
\text { (total) }\end{array}$ & $\begin{array}{l}\text { Rosenstein et al. (Rosenstein et } \\
\text { al., 2021) (SRR11488976, } \\
\text { SRR11488977) }\end{array}$ & $\begin{array}{l}\text { From infected } \\
\text { human skin }\end{array}$ & Human \\
\hline BSPyV1 & $\begin{array}{l}\text { Short-RNAseq } \\
\text { (total) }\end{array}$ & $\begin{array}{l}\text { Identified by Schmidlin et al. } \\
\text { (Schmidlin et al., 2021) } \\
\text { (SRR5958578) }\end{array}$ & $\begin{array}{l}\text { From whole } \\
\text { scorpion }\end{array}$ & $\begin{array}{l}\text { C. } \\
\text { sculpturatus }\end{array}$ \\
\hline
\end{tabular}

\section{Tumor samples}

472 The BKPyV-associated bladder cancer is sample TBC03 that has been described

473 (Starrett et al., 2021). This sample is stranded, short-RNAseq (total).

475 Merkel cell carcinoma samples: Sections of tissue were isolated from patient-derived

476 tumor biopsies and suspended in RNAlater (Thermo Fischer) until further processing. 
477 RNA and DNA was extracted from each section via the AlIPrep DNA/RNA kit (Qiagen).

478 Isolated RNA and DNA were each sequenced (PE150) on the NovaSeq 6000 platform

479 (Illumina) for a depth of $50 \mathrm{M}$ reads or 60x genomic coverage per sample, respectively

480 (Novogene). RNAseq data are unstranded, short-RNAseq (polyA).

481

482

SV40 infection and RNA extraction

483 BSC40 cells (ATCC CRL-2761) were seeded on $150 \mathrm{~mm}$ dishes at $5.37^{*} 10^{6}$ cells per

484 plate - about $70 \%$ confluence. After waiting 4 hours for the cells to adhere, cells were

485 infected with SV40 at MOI 1 as previously described (Tremblay et al., 2001) with slight

486 modification. In brief, maintenance media was removed, and each 150mm dish was

487 inoculated with $6 \mathrm{~mL}$ of virus stock diluted in DMEM $+2 \%$ FBS. Infection was allowed to 488 proceed at $37^{\circ} \mathrm{C}, 5 \% \mathrm{CO} 2$ for one hour, with the plates rocked every 15 minutes to 489 ensure adequate coverage of the solution over the cell monolayer. At the end of this 490 period, DMEM + 2\% FBS was added to a final volume of $25 \mathrm{~mL}$ per $150 \mathrm{~mm}$ dish. Each

491 dish was then incubated at $37^{\circ} \mathrm{C}, 5 \% \mathrm{CO} 2$ for 48 hours. RNA was extracted using the 492 QIAGEN RNeasy Mini Plus Kit (QIAGEN 74134). This total RNA was then subjected to 493 Nanopore direct RNA sequencing and Illumina total- and polyA-RNA sequencing as 494 described below.

496 BKPyV infection and RNA extraction

497 Archetype and rearranged BKPyV (Dik and Dunlop, respectively) were purified and 498 titrated as described (Jiang et al., 2009b). RPTE-hTERT cells (Zhao and Imperiale, 
499

500

501

502

503

504

505

506

507

508

509

510

511

512

513

514

515

516

517

518

519

520

521

2019) were plated in 6-well plate and prechilled for $15 \mathrm{~min}$ at $4^{\circ} \mathrm{C}$ and infected with Dik or Dunlop at a MOI of 1 and 0.5 fluorescence-forming unit (FFU)/cell, respectively. The cells were incubated at $4^{\circ} \mathrm{C}$ for $1 \mathrm{~h}$ with gentle shaking every $15 \mathrm{~min}$. The virus was removed and fresh REGM medium was added to the cells. Dik and Dunlop infected cells were collected at $120 \mathrm{hpi}$ and $96 \mathrm{hpi}$, respectively. Total RNA was extracted using the Direct-zol RNA MiniPrep kit (ZYMO Research, USA). This total RNA was then subjected to Nanopore direct RNA sequencing and Illumina total- and polyA-RNA sequencing as described below.

\section{MPyV infection and RNA extraction}

C57 mouse embryo fibroblasts (ATCC SCRC-1008) were plated on a $150 \mathrm{~mm}$ dish at $40 \%$ confluence. After several hours of growth, the typical DMEM $+10 \%$ FBS media was replaced with serum free DMEM. The next day, the crude viral stock was thawed at $37^{\circ} \mathrm{C}$, incubated at $45^{\circ} \mathrm{C}$ for 20 minutes to facilitate the final liberation of virus into the supernatant, and cell debris removed from the viral stock with centrifugation. The prepared virus stock was then diluted 1:10 with an absorption buffer consisting of HBSS with $10 \mathrm{mM}$ HEPES, $1 \% \mathrm{FBS}$, at $\mathrm{pH}$ 5.6. Media was removed from the target cells, and $6 \mathrm{~mL}$ of diluted virus in absorption buffer was added. Infection was allowed to proceed at $37^{\circ} \mathrm{C}, 5 \% \mathrm{CO} 2$ for one hour, with the plates rocked every 15 minutes to ensure adequate coverage of the solution over the cell monolayer. At the end of this period, the absorption buffer was removed and DMEM $+2 \%$ FBS was added to a final volume of $25 \mathrm{~mL}$ per $150 \mathrm{~mm}$ dish. Cells were inoculated for 28 hours at $37^{\circ} \mathrm{C}, 5 \% \mathrm{CO}$, after which RNA was extracted using TRIzol (ThermoFisher 15596026) according to the 
522 manufacturer's instructions. This total RNA was then subjected to Nanopore direct RNA

523 sequencing as described below.

524

525 The virus stock used here was kindly provided by the lab of Robert Garcea. This virus

526 stock (viral strain NG59RA) was a crude supernatant from MPyV-infected cells originally

527 generated by the lab of Thomas Benjamin on 02/08/2011 and was of unknown titer.

528 This stock was subjected to a total of three freeze-thaw cycles before use.

\section{SV40 polysome profiling}

531 BSC40 cells were plated on $4150 \mathrm{~mm}$ dishes at $60 \%$ confluence. After waiting 4 hours

532 for the cells to adhere, cells were infected with SV40 at MOI 1 as reported above. At 44

533 hours post infection cell culture media was replaced with media containing $100 \mathrm{ug} / \mathrm{mL}$

534 cycloheximide and incubated for 5 minutes. Plates were placed on ice, media

535 discarded, and cells were scraped into PBS containing 100ug/mL cycloheximide. Cells

536 were spun down, the PBS discarded, and cells were lysed in a lysis buffer containing

537 10mM Tris (pH 8), 100mM KCl, 10mM MgCl2, 2mM DTT, 1\% Triton X100, 100ug/mL

538 cycloheximide, and 1unit/uL SUPERase RNase inhibitor (Thermo AM2694). Lysates

539 were incubated on ice for 20 minutes with intermittent tapping, and then spun at

$54010,000 \mathrm{~g}$ for 10 minutes at $4^{\circ} \mathrm{C}$. The supernatant was loaded onto a $10-55 \%$ sucrose

541 gradient followed by ultracentrifugation (Beckman Coulter Optima XPN-100

542 ultracentrifuge) at $32,500 \times \mathrm{rpm}$ at $4{ }^{\circ} \mathrm{C}$ for 80 minutes in the SW41 rotor. Gradients

543 were prepared with a gradient mixer and pump. Samples were separated by density 
544 gradient fractionation system (Biocomp Piston gradient fractionator IP). RNA was

545 extracted from reserved input ("whole-cell") lysate, as well as the polysome fraction

546 using TRIzol. Equal volumes of each fraction containing heavy polysomes (>2) was

547 pooled prior to extraction (Lee et al., 2020).

Western blotting

550 Infection of wildtype Dik and two Dik mutants in RPTE-hTERT cells was performed as

551 mentioned above. Protein samples were harvested in E1A buffer with protease and

552 phosphatase inhibitors, electrophoresed, transferred, and probed with large tumor

553 antigen antibody (pAb416) as previously described (Zhao and Imperiale, 2019).

\section{RNA sequencing}

555 The concentration of total RNA was determined using the Qubit Fluorometer with the 556 Qubit RNA HS Assay Kit (ThermoFisher Q32852). RNA quality was then assessed on 557 an Agilent Bioanalyzer and the RNA 6000 Pico Kit (Agilent 5067-1513). PolyA RNA was

558 isolated using the NEBNext Poly(A) mRNA Magnetic Isolation Module (NEB E7490S)

559 with an input of 5ug of total RNA - for SV40 and BKPyV Dunlop, up to 8 total reactions

560 were used to yield sufficient polyA RNA (500ng) for subsequent protocols. In the case of

561 MPyV, due to limited amounts of total RNA, three reactions were used to yield roughly

562 100ng of polyA RNA. PolyA RNA concentration was determined again using the Qubit

563 RNA HS Assay Kit (ThermoFisher Q32852). PolyA RNA was then concentrated to 9uL

564 using a centriVap. 
$500 \mathrm{ng}$ of polyA RNA (or, in the case of MPyV, 100ng) in 9uL was then processed using

567 the Nanopore Direct RNA sequencing kit (SQK-RNA002). Resultant libraries were

568 sequenced for up to 24 hours on a MinION using an R9.4.1 flow cell.

570 In the case of polysome profiling: extracted RNA from the input and polysomes were

571 separately subjected to 5 reactions each of the NEBNext Poly(A) mRNA Magnetic

572 Isolation Module using 5ug RNA input per reaction. All resultant polyA RNA was then

573 processed using the Nanopore Direct RNA sequencing kit (SQK-RNA002). Resultant

574 libraries were sequenced for up to 24 hours on a MinION-Mk1C using an R9.4.1 flow

575 cell.

576

577 Illumina total RNA sequencing and polyA RNA sequencing of SV40-, BKPyV Dunlop-, 578 and BKPyV Dik-infected cells was conducted by Novogene Corporation Inc. The QC for 579 the RNA samples was performed using Qubit and Bioanalyzer instruments. Libraries 580 were then prepared using NEBNext Ultra II with RiboZero Plus kit (for short-RNAseq 581 (total)) and NEBNext Ultra II with PolyA Selection kit (for short-RNAseq (polyA)). Both

582 library approaches are strand-specific. Library quality and concentration was assessed 583 with Labchip and qPCR. Libraries were sequenced on NovaSeq6000 using PE150 584 sequencing.

586 PacBio SMRT sequencing of SV40 and BKPyV Dunlop was conducted by the Georgia 587 Genomics and Bioinformatics Core. Each sample was subjected to IsoSeq library 
588 preparation and sequenced on an individual 8M SMRT cell for 26 hours on a Sequel-II

589 machine.

590

\section{Initial Sequence Processing}

592 Raw Nanopore dRNAseq reads from standard SV40, BKPyV Dunlop, and MPyV

593 infections were basecalled with Guppy version 4.2.2 with the following command:

594 guppy_basecaller -i fast5 -s basecalled --flowcell FLO-MIN106 --kit SQK-RNA002 -r --

595 trim_strategy rna --reverse_sequence true --u_substitution true --

596 cpu_threads_per_caller 10

598 Raw Nanopore dRNAseq reads from polysome profiling of SV40 transcripts were

599 basecalled on a MinION-Mk1C using MinKNOW version 21.02.2.

600

601 PacBio SMRTseq subreads were processed using ccs (version 6.0.0). Full-length,

602 nonchimeric reads were then generated using the lima (version 2.0.0) and Isoseq3

603 (version 3.4.0) packages provided by PacBio.

604

605 Stranded Illumina short-RNAseq (total) and short-RNAseq (polyA) reads were

606 processed in the following way: Files containing read 1 (R1) and read 2 (R2) were

607 trimmed and adapters removed using Trim Galore! (Krueger, 2016). Next, reads in R1

608 files were reverse complemented to orient the reads correctly relative to the transcript of 
609 origin, and all read headers in the R1 and R2 files were labeled with "_1" or “_2”

610 respectively. The R1 and R2 files were then concatenated. This Illumina processing

611 pipeline is available in process_illumina.nf.

613 The MCC tumor RNAseq assessed in this manuscript were short-RNAseq (polyA) that

614 were NOT stranded. This means that the strand of origin of each read is unknown. To

615 address this uncertainty, the complement AND reverse complement of both R1 and R2

616 were concatenated into the final FASTQ file. As described below in the section

617 "Processing of short-read short-RNAseq (total) and short-RNAseq (polyA) span files",

618 future processing kept the most-likely alignment strand for each read.

Sequence Alignment and Processing

621 Most long-read sequencing data and Illumina sequencing data were aligned to the

622 appropriate viral genome using Minimap2 (Li, 2018). The exceptions are the short-

623 RNAseq (total) JCPyV data from Assetta et al. (Assetta et al., 2016) and the HPyV7

624 data from Rosenstein et al. (Rosenstein et al., 2021) - these samples contained

625 sequencing reads of $101 \mathrm{bp}$ or shorter and were instead mapped with STAR (Dobin et

626 al., 2013). All non-primary alignments were discarded. Sequence alignments in BAM

627 format were then converted to BED using bedtools (Quinlan and Hall, 2010). Here,

628 bedtools considers any Minimap2- or STAR-called intron ("N" cigar flag) as an intron to

629 split alignment segments. Parameters for alignment and bed conversion can be found in

630 minimap2.sh and star.sh. 
632 To capture transcripts that originate from a pre-mRNA that circled the viral genome

633 more than once, and therefore contain repetitive sequences, all alignments were

634 conducted against concatenated copies of the viral genome. In the case of short-read

635 short-RNAseq (total) and short-RNAseq (polyA), the reference consisted of two

636 concatenated copies of the viral genome. For long-read dRNAseq and SMRTseq, the

637 reference consisted of twenty concatenated copies of the viral genome.

639 Because the references consisted of multiple copies of the same viral genome, mapped

640 reads were assigned to a random copy of the genome. Therefore, all reads in resultant

641 BED files were "slid" such that they started in the first genome copy of the reference

642 using bed_slide_wraparound_reads.py.

644 All reference genomes can be found in resources/ref directory of the associated github 645 repository. All references used contain the PyV late region at the start/5' end of the 646 reference on the "+" or sense strand, with the early region on the antisense or "-" strand.

647 The concatenated references are based on the following reference genomes collected 648 from NCBI, with any modifications listed:

$649 \quad$ - SV40: NC_001669.1. The first 100 nucleotides were moved to the end of the 650 sequence.

$651 \quad-\quad$ BKPyV: KP412983.1

652

- JCPyV: NC_001699.1 
- MPyV: NC_001515.2. The sequence was reverse-complemented to orient the late region towards the start of the reference.

- MCPyV: NC_010277.2

656

- HPyV7: NC_014407.1

657

- $\quad$ BSPyV1: LN846618.1

659 Next, a span file was generated from each slid BED file using bed_to_span.py. This

660 script splits each read into "spans", where each span is an exon or an intron with all

661 positions relative to the viral genome. The introns are defined by the Minimap2- or

662 STAR-called introns ("N" cigar flag) as mentioned above. All regions between the start

663 and end of the reads that are not introns were called as distinct exons. Transcripts were

664 clustered into transcript classes based on introns as discussed below. A "tidy" output

665 span file was then generated that contains the name, strand, and transcript class of a

666 given read, with separate lines for the start and end of each span (e.g., exon or intron)

667 within the sequencing read.

\section{Alignment of repetitive regions}

670 Reads that originate from a transcript that circles the genome more than once can be

671 detected because there is one or more repetitive regions within the read. Alignment

672 against multi-copy reference genomes (20 copies in the case of dRNAseq and

673 SMRTseq) as described above sufficiently captured most of these transcripts, with

674 some exceptions. First, BKPyV SMRTseq data had a poor alignment rate of the leader 
675 exon in late WA transcripts - this means that WA transcripts are underrepresented in the

676 BKPyV SMRTseq data. Second, alignment of superT and superT* transcripts from

677 SMRTseq and dRNAseq data was generally poor, with the repetitive region often failing

678 to map via Minimap2. Potential superT and superT* reads in dRNAseq and SMRTseq

679 data were identified through assessment of BAM files following mapping. Early reads

680 that contain a CIGAR flag showing an insertion of 100 bases or more were flagged, and

681 up to 50 of these transcripts were manually investigated through online BLASTN

682 (Johnson et al., 2008) against the viral reference genome. Reads supporting superT in

683 SV40 dRNAseq data, superT* in SV40 SMRTseq data, and superT in BKPyV SMRTseq

684 data were initially missing from Minimap2 alignments but were identified via this

685 approach. One transcript of each type was then repaired upon data import to R such

686 that these transcripts are represented in downstream visualizations - these actions are

687 clearly marked in UTILS_import_data.R. Thus, superT and superT* in SMRTseq and

688 dRNAseq data are underrepresented in abundance plots (Figure S3D, S4D) and read

689 pileups (Figure S6, S8) compared to their actual abundance in the cell due to these

690 alignment challenges.

\section{Generation of transcript classes}

693 Transcript classes were generated during processing of BED files using

694 bed_to_span.py. Each transcript class consists of sequencing reads that contain the

695 same combination of introns. The transcript class number is based on the abundance of

696 transcripts within a transcript class - e.g., transcript class 1 contains more transcripts

697 than transcript class 2, and so on. Transcript class generation is similar for both long- 
698 and short-read sequencing data, although short reads usually (but not always) tend to

699 contain a maximum of one intron. Notably, transcript class assignment is independent of

700 the transcript start and end positions, meaning that there can be heterogeneity of

701 transcript start and end positions within a transcript class. For all SMRTseq and

702 dRNAseq data, for a transcript class to be generated all introns contained within the

703 transcript class were required to be supported by at least 5 junction-spanning reads

704 within a short-RNAseq (total) dataset. For SV40 and BKPyV Dunlop SMRTseq and

705 dRNAseq data, the short-RNAseq (total) data was generated from RNA from the same

706 extraction. SV40 dRNAseq replicate 2 was corrected with the short-RNAseq (total) data

707 from the first SV40 replicate. For the MPyV dRNAseq data, short-RNAseq (total) data

708 from Garren et al. was used. If a transcript contained an intron that was not supported

709 by at least 5 junction-spanning reads in the Illumina dataset, it was discarded. We opted

710 to use this filtering strategy rather than implementing long-read correction because

711 correction algorithms were unable to cope with wraparound transcripts.

713 There were limited circumstances where dRNAseq or SMRTseq transcript classes were

714 removed manually during processing - this occurred to four transcript classes that made

715 it through filtering. In these circumstances, alignments were deemed to be artifactual

716 due to Minimap2 alignment errors. These instances are clearly programmatically

717 marked in UTILS_import_data.R with specific rationale for each action. 
720 All splice or intron positions marked in any figure or table of this manuscript are $\mathbf{0}$ -

721 indexed positions of the intron. To convert these coordinates to the 1-

722 indexed/absolute position of the intron on the viral genome, add 1 to the intron start

723 position. For example, for the intron 276-1600, viral genome nucleotide \# 277 is the first

724 nucleotide within the intron, and viral genome nucleotide \# 1600 is the last nucleotide

725 within the intron.

726

727 Processing of short-RNAseq (total) and short-RNAseq (polyA) span files

728 The majority of the short-read RNAseq data investigated here used a strand-specific

729 sequencing strategy (except for the MCC tumor RNAseq). With this strategy, the strand

730 of origin for the transcript yielding each read is known, and a read can be correctly

731 assigned to the sense ("+" / late) or antisense ("-" / early) strand. However, a fraction of

732 transcripts can be inaccurately stranded due to artifacts during library preparation.

733 When there were many more late reads than early reads in a short-read dataset, a

734 prohibitive fraction of "early" reads would be reads from late transcripts that were

735 incorrectly stranded due to this artifact. To address this issue, short reads that aligned

736 to the + strand were required to either start or end within the late region (defined as the

737 first $1 / 2$ of the genome), and short-reads that aligned to the - strand were required to

738 either start or end within the early region (defined as the second $1 / 2$ of the genome). 
741 For Figure 1 and all supplementary figures, a SV40 or BKPyV transcript was identified

742 and assigned a transcript ID if it was at least $0.1 \%$ of early or late strands in dRNAseq

743 or SMRTseq data with one exception - SV40 transcript L8 had been previously

744 identified and was kept despite being at only $0.06 \%$ abundance. Existing transcript

745 names, where available, were taken from relevant studies (Abend et al., 2009; Good et

746 al., 1988; Seif et al., 1979; Zerrahn et al., 1993). This assignment occurred from the

747 span files, meaning that all sequencing reads in question were previously required to

748 contain introns that were supported by at least 5 short-RNAseq (total) junction-spanning

749 reads. For SV40, for which there was two dRNAseq replicates, identification of a

750 sequencing read at $0.1 \%$ or greater in just one replicate was sufficient.

751

752 Transcript IDs (e.g., E1, E2, E3..., L1, L2, L3,...) consist of the kinetic class (E: Early, or

753 L: Late) of the identified transcript followed by an integer value in ascending order of

754 abundance. This abundance value was calculated by ordering the transcripts in order of

755 the maximum observed relative abundance in dRNAseq or SMRTseq data.

757 Of note, the relative abundance of transcripts between dRNAseq and SMRTseq data is

758 skewed by distinct read-length biases between the two approaches. The dRNAseq

759 approach has a 3' bias and a bias towards shorter transcripts, while SMRTseq library

760 preparation resulted in preferential sequencing of transcripts closer to $\sim 2500 \mathrm{bp}$ in

761 length. Resultant differences in the length of aligned reads can be seen in Figure S1C.

762 The TSS distribution of SV40 late transcripts varies between transcript classes, while 
763 the late TSS distribution tends to be similar across transcript classes in BKPyV (Figure

764 S2A).

765

766 Calculation of sequencing coverage

767 To determine the sequencing coverage for each sample (as in Figure 1C), BAM files

768 from alignment were "slid" such that all transcripts must start in the first genome copy of

769 the reference using bam_slide_wraparound_reads.py, in a similar manner as the beds

770 were slid as described above. Forward and reverse strand reads were split, and the

771 depth was calculated using the command `samtools depth -aa -d0` separately for

772 forward and reverse reads. These processing steps are present in bam_coverage.nf.

773 During plotting, the coverage for each strand was normalized to the maximum coverage

774 at any position (e.g., the maximum coverage of the late and early strands was set to 1).

775

776 Watch plots

777 Each panel of a watch plot represents information for a single transcript class. The

778 center "arms" of these plots are histograms detailing the distribution of start (blue) and

779 end (red) positions for the transcripts within the transcript class. These histograms are

780 normalized to the highest abundance position. The outer ring of each watch plot shows

781 the viral ORF map. Each inner grey ring indicates the number of genomes spanned - all

782 transcripts are displayed moving outwards from the center. Red segments indicate the

783 exons of each transcript class. The first exon starts on the most-inner grey ring at the

784 most common transcript start site for the transcript class, and the last exon ends on the 
785 most-outer grey ring at the most common transcript end site for the transcript class. The

786 3' end of the transcript is indicated by the red arrow at the end of the last exon. Thus,

787 the transcripts spiral outwards from the center in the direction of the red arrow. Figure 4

788 contains a schematic key describing watch plots.

789

790

Read pileup plots

791 Each square/rectangular panel of a read pileup plot shows the reads present in a single

792 transcript class. The arrows at the top of each panel indicate the viral ORF map, with

793 dashed lines indicating the end of each genome copy. Next, the lines indicate

794 histograms of the transcript start (blue) and end (red) sites for the transcripts within the

795 transcript class. Below the x-axis, each row indicates a single sequencing read. The

796 spans in red indicate the exons inferred from a sequencing read, while the spans in pink

797 indicate the introns/splice junctions. Sometimes the distribution of transcript end

798 positions for a transcript class can be obscured by the thickness of the transcript lines -

799 the histograms should always be consulted to assess abundance.

800

801 For SV40 dRNAseq watch and pileups: There were two SV40 dRNAseq replicates.

802 Watch plots and read pileups are based on replicate 1, although missing transcripts that

803 were identified in replicate 2 but not 1 were also plotted.

804

805 Short-read intron plots (Figure S9, S11) 
806 In these plots, lines indicate specific introns. The upper and lower horizontal arrows

807 indicate the viral ORF map - often, these ORF maps will indicate two concatenated viral

808 reference genomes. The circles above or below each ORF map indicate the percentage

809 of early or late introns that fall at each genome position. Early introns and percentages

810 are colored red, while late introns and percentages are colored blue.

\section{2 polyA tail length}

813 polyA tail length was determined from dRNAseq data using the 'polya`command of

814 Nanopolish (Loman et al., 2015). To determine the polyA distribution of host transcripts,

815 sequencing reads were aligned to the human GRCh38 (for BKPyV samples), $C$.

816 Sabaeus (for SV40 samples), or mouse (for MPyV) cDNA transcriptomes downloaded

817 from ensembl. Only reads with a Nanopolish QC tag of "PASS" were considered for

818 downstream polyA tail length analyses.

820 The dRNAseq library preparation included the addition of the "RNA Control Standard"

821 (RCS), which is a synthetic RNA based on yeast ENO2 containing a 30-adenine polyA

822 tail. dRNAseq samples were mapped against ENO2 to assess the polyA tail length

823 distribution of this control. 
825 The cumulative incidence of transcript termination (Figure S10D) was calculated by

826 determining, for each early read, how far the read's transcript end site is from the

827 canonical polyA site position for each virus.

829 Polysome profiling analysis

830 To determine the ribosome occupancy of host genes, dRNAseq reads were aligned to

831 the C. Sabaeus cDNA transcriptome downloaded from ensembl. The number of reads

832 mapped to each transcript was extracted with `samtools idxstats`. Transcripts were

833 filtered to include only those with at least 10 reads in both polysome and input fractions.

834 The normalized abundance of each transcript in each fraction was defined as (\# of

835 mapped reads)/(total number of virus and host mapped reads). Ribosome occupancy of

836 each transcript was determined as (normalized abundance in polysome)/(normalized

837 abundance in whole-cell), where a value of $>1$ indicates preferential translation.

839 Ribosome occupancy of individual viral transcripts could not be calculated because of

840 increased rates of transcript truncation in the polysome fraction compared to the whole-

841 cell fraction. This was indicated by a nearly doubled proportion of unspliced reads with

842 premature 5' ends in the polysome fraction compared to the whole-cell fraction, and

843 likely indicates transcript degradation during sucrose centrifugation or fraction collection.

844 Because viral transcripts are mostly identical and vary largely at a 5' splice site,

845 elevated transcript truncation decreased the observed abundance of individual viral 
846 transcripts in the polysome fraction and make ribosome occupancy calculations for

847 individual viral transcripts unreliable.

MCC440 superT PCR and sanger sequencing

850 Anchored poly-dT primers (Life Technologies) were used for specific reverse-

851 transcription of full-length mRNA into cDNA. Primers were designed to uniquely amplify

852 the super-LT junction through exploitation of repetitive sequences. Primer sequences

853 were as follows (5' -> 3'); Forward: CTGGACTGGGAGTCTGAAGC, Reverse:

854 ACCCCTCCTCCATTCTCAAGA. Q5 polymerase (NEB) with standard reaction

855 conditions was used for amplification.

\section{Generation of integrated PyV structures and viral variant calling}

858 Tumor WGS was aligned against a fusion reference genome containing hg38 and

859 Merkel cell polyomavirus (NC_010277) using bowtie2 with default parameters.

860 Integrated virus assembly graphs and annotations were generated using Oncovirus

861 tools (https://github.com/gstarrett/oncovirus_tools). Assembly graphs were then

862 manually interpreted to create linear integration structures for PyV-associated MCC.

864 Point mutations were called in the PyV genomes using lofreq with default parameters 865 (https://csb5.github.io/lofreq/) (PMID: 23066108). Lofreq output was functionally 866 annotated with SnpEff (http://pcingola.github.io/SnpEff/) (PMID: 22728672) using the 
867 relevant GenBank gene annotations for the above genomes. Variants were plotted out

868 in $\mathrm{R}$ with the ggplot2 package.

869

870

871 List of abbreviations

872 APA - Alternative polyadenylation

873 BKPyV - BK Polyomavirus

874 BSPyV1 - Bark scorpion polyomavirus 1

875 dRNAseq - Nanopore direct RNA sequencing

876 HPyV7 - Human polyomavirus 7

877 JCPyV - JC Polyomavirus

878 LT - Large T antigen

879 MCC - Merkel cell carcinoma

880 MCPyV - Merkel cell polyomavirus

881 MPyV - Murine polyomavirus

882 MT - Middle T antigen

883 ORF - Open reading frame

884 SMRTseq - PacBio SMRT sequencing 
885 ST - Small T antigen

886 SV40 - Simian virus 40

887 TES - Transcript end site

888 TSS - Transcript start site

889 PVAN - Polyomavirus-associated nephropathy

890 PyV - Polyomavirus

891

892

893 Declarations

894 Competing interests

895 M.M. receives research support from Bayer, Janssen, Ono; consults for Bayer, Interline,

896 Isabl; and receives patent royalties from Labcorp and Bayer. J.A.D. has received

897 research support from Rain Therapeutics, Inc. and is a consultant for Rain

898 Therapeutics, Inc. and Takeda, Inc.

$900 \quad$ Funding

901 This work was supported in part by the US Public Health Service grants R35CA232128

902 and P01CA203655 and by the Bridge Project, a partnership between the Koch Institute

903 for Integrative Cancer Research at MIT and the Dana-Farber/Harvard Cancer Center to 
904 J.A.D.; a Cancer Grand Challenges OPTIMISTICC team award (C10674/A27140) and

905 by National Cancer Institute grant R35CA197568 to M.M.; R01 Al060584 and R21

906 Al147155 to M.J.I; CD \& SV were supported by R35GM134944; GJS is supported by

907 the NIH Intramural Research Program.

908

909 Authors' Contributions

910 Conceptualization - J.N., M.M., J.A.D.

911 Data curation - J.N.

912 Formal analysis - J.N.

913 Funding acquisition - M.M, J.A.D., M.J.I., S.V., G.J.S.

914 Investigation - J.N., W.Z., T.C.F., C.D.

915 Methodology - J.N., M.M., J.A.D.

916 Project administration - J.N.

917 Resources - J.N., M.M., J.A.D., T.C.F., W.Z., M.J.I, S.V., C.D.

918 Software - J.N.

919 Supervision - M.M., J.A.D.

920 Validation - J.N.

921 Visualization - J.N.

922 Writing, Original draft - J.N., M.M., J.A.D. 
923 Writing, review \& editing - J.N., W.Z., T.C.F., C.D., S.V., G.J.S., M.J.I., M.M., J.A.D.

\section{Acknowledgements}

926 We thank Katie Vicari (www.KatieRisVicari.com) for her work illustrating Figure 1A, 2, 3,

927 and S12B. Figure 1F was made in BioRender. We thank Mary O'Reilly with the Broad

928 Research Communication Lab for helpful discussions on figure design. We thank

929 Robert Garcea and Kimberly Erickson for kindly providing stocks of MPyV. Portions of

930 this research were conducted using the O2 High Performance Computing Cluster,

931 supported by the Research Computing Group at Harvard Medical School. Portions of

932 this work utilized the computational resources of the NIH HPC Biowulf cluster

933 (http://hpc.nih.gov).

935 Figure legends

936 Figure 1 - RNA sequencing expands known SV40 and BKPyV transcript diversity.

937 A. Overview of experimental procedures. Cells were infected with a polyomavirus, 938 and RNAs extracted. RNA was sequenced using long-read (Nanopore dRNAseq 939 and PacBio SMRTseq) and short-read (Illumina short-RNAseq (total) and short940 RNAseq (polyA)). Transcripts were analyzed, and the impact of observed splice 941 events on viral open reading frames was assessed.

942 B. Mechanism of transcript clustering in this study. Transcripts were aligned to the viral genome and grouped into transcript classes based on the presence of 
shared introns. Thus, within a transcript class there may be variation in the exact transcript start and end positions. This clustering strategy was used for both longand short-RNAseq data.

C. Viral RNA sequence coverage for SV40 and BKPyV as determined from dRNAseq, SMRTseq, and short-RNAseq (total) data. The $Y$ axis indicates the scaled coverage, with $\mathrm{X}$ axis indicating the position on the viral genome. Coverage for late transcripts (mapping to the + strand) is above the $\mathrm{x}$ axis, while coverage for early transcripts (mapping to the - strand) is below the $\mathrm{x}$ axis. Coverage is scaled separately for each strand such that the maximum observed coverage for each strand is 1 . Arrows at the top of the plot indicate the positions of viral genes.

D-E. UpSet plot indicating the overlap between existing transcript annotations,

956 dRNAseq data, and SMRTseq data for SV40 (D) and BKPyV Dunlop (E). Bars

957 indicating overlap with existing transcript annotations are black, while those

958 indicating no overlap with existing annotations are blue. These blue bars indicate the 959 number of novel, unannotated transcripts identified. infected with SV40. Cells were lysed, and a portion of the lysate was subjected to dRNAseq (representative of the RNA content of the whole cell). The remaining lysates was centrifuged through a sucrose gradient, after which fractions containing RNA associated with two or more ribosomes were pooled and subjected to dRNAseq. 
G. Relative abundance of SV40 early and late transcripts in the whole-cell and polysome fractions of SV40-infected cells. Y-axis indicates the percentage of early or late transcripts and is log scale. X axis indicates each transcript, with black dots indicating each transcript's whole-cell relative abundance and red dots indicating each transcript's polysome relative abundance.

971

Figure 2 - Annotated and novel SV40 transcripts.

A. Transcripts are shown relative to the viral genome. Each line is a viral transcript, with red lines indicating exons and dashed blue lines indicating introns. Spokes indicate the positions of common splice donors and splice acceptors. Transcripts that were annotated prior to this study are on a yellow background, and novel transcripts are on a while background. Wraparound transcription that results in multiple copies of a region is annotated with double lines, and the number of copies is indicated in parentheses. The line labeled "pA" indicates the approximate position of the polyA signal sequence. with red lines indicating exons and dashed blue lines indicating introns. Spokes indicate the positions of common splice donors and splice acceptors. Transcripts that were annotated prior to this study are on a yellow background, and novel transcripts are on a while background. Wraparound transcription that results in multiple copies of a region is annotated with double lines, and the number of 
copies is indicated in parentheses. The line labeled "pA" indicates the approximate position of the polyA signal sequence. transcript classes in dRNAseq data from SV40 (A), BKPyV Dunlop (B), and MPyV (C). The outer ring of each watch plot indicates the position of the viral ORFs. The inner arms are histograms detailing the distribution of transcript starts (in blue) and ends (in red) for transcripts within each transcript class. The red segments indicate exons. Transcripts start in the innermost ring - a second or third ring indicates that the pre-mRNA that generated the transcript must have circled the viral genome multiple times. The 3' end of the transcript and the direction in which these plots are oriented is indicated by the red arrow at the end of the last exon segment. The red exon segments start at the most common transcript start site within the transcript class, and end at the most common transcript end site within the class. The watch plot key shows an example of the path of the pre-mRNA for SV40 transcript class L6_I. genome lengths in SV40, BKPyV Dunlop, and MPyV dRNAseq data. subsequent wraparound, was identified in Illumina short-RNAseq (total) data. The intron in question is plotted as a black line in this plot, with the $\mathrm{x}$ axis 
1010

1011

1012

1013

1014

1015

1016

1017

1018

1019

1020

1021

1022

1023

1024

1025

1026

1027

1028

1029

1030

1031

1032

indicating the genomic position of the intron. The top late wraparound transcript for each virus was plotted. The gene map indicates the approximate gene position and is accurate for SV40 - the exact position of the viral genes varies between viruses. Percentages indicate the percentage of late junction-spanning transcripts that support the plotted wraparound leader-leader junction.

F. Schematic illustrating how leader-leader wraparound transcription can be detected from short read short-RNAseq (total). Leader-leader splicing can be seen as a repetitive exon in watch plots from long-read RNAseq data. Ultimately, there was an original processed mRNA in the cell that contained two tandem leader sequences. When this transcript of origin is sequenced via short read sequencing, reads will be generated across its length. A minority of these reads will span the leader-leader junction, and mapping against the viral reference genome can be used to uncover leader-leader splicing.

Figure 5 - Detection of novel, conserved splicing events that expand PyV coding capacity.

A-D. Schematics illustrating identified ORFs. Each row is a reading frame (except for ST and the LT $1^{\text {st }}$ exon, which are in the same frame), and unannotated amino acids are represented by grey boxes. The measured intron is indicated by the red arrow. Colored ORFs are annotated, while grey ORFs are unannotated. Percentages on the right side of the figure are the percentage of spliced viral transcripts on the same strand as determined from short-read short-RNAseq (total) data. Numbers after each virus name indicate the transcript class within each short-RNAseq (total) dataset. The measured intron is indicated by the red arrow. 
E. Schematics detailing BKPyV Dik isolates used for querying the existence of superT.

1053

A) ST2: This ORF is generating from a splicing event that uses the LT first exon donor and an acceptor within the ST ORF. In HPyV7 and BKPyV Dunlop, the splice lands in frame and results in an internal deletion within ST. In MPyV and MCPyV the splice lands out of frame, resulting in an ORF that contains the $\mathrm{N}$-terminal region of ST and novel amino acids at the $\mathrm{C}$ terminus.

B) MT: MPyV encodes a MT following splicing connecting the end of the ST ORF with an ORF in an alternate frame of the LT second exon. In BKPyV, a similar splice occurs connecting ST with an MT-like ORF in an alternative frame of the LT second exon.

C) VP1X: JCPyV encodes two VP1X ORFs generated by splicing within VP1 and landing in an alternative frame of VP1, or earlier in the late region due to wraparound transcription. While predominant in JCPyV, VP1X is likewise present in many other PyV.

D) superT: The superT-specific splice utilizes the splice donor canonically associated with truncated T antigens such as $17 \mathrm{kT}$ in SV40 and truncT in BKPyV. Due to wraparound transcription, a LT second exon acceptor is available to the 3 ' of this donor and acts as the acceptor. For the superT ORF to form, an initial LT splice is required. Ultimately, superT contains a duplication in part of the LT second exon that includes the RB-binding LxCxE motif. BKPyV WT is wild type virus. M1 contains a LT intron that has been replaced with an intron from the plasmid $\mathrm{pCl}$. Both WT and M1 are expected to generate LT and superT 
1055 of expected sizes. M2 has a completely removed LT intron, and the $\mathrm{pCl}$ intron is located

1056 directly 5' of the LT ORF. M2 is expected to encode LT of expected size, but a larger

1057 superT variant due to incorporation of a second copy of the LT first exon.

1058 F. Western blot of cells infected with BKPyV Dik WT, M1, or M2 and probed with an

1059 antibody reactive against LT. The lower molecular weight band is LT, and the higher

1060 molecular weight bands are consistent with superT.

Figure 6 - Detection superT-encoding transcripts in PyV-associated cancers

A. Schematic detailing the generation of superT during lytic infection as compared to from integrated virus in cancer. During viral infection, the RNA polymerase can circle the viral genome multiple times, resulting in a pre-mRNA that can be spliced to generate superT. In the case of host integration, a polyomavirus can be integrated in tandem copies such that a pre-mRNA is generated with more than one copy of the viral early region. This pre-mRNA can be similarly spliced to generate a superT transcript.

B. Heatmap indicating the abundance of the superT, ST, and LT introns from RNAseq data from two replicates of a BKPyV-positive bladder cancer and six MCPyV-associated MCCs. Percentages indicate the percentage of spliced early viral reads for each sample. The splice measured in each row is indicated by the red arrow in the schematics on the right side of the figure. 
A. The number of reads for all datasets studied here. For long-read dRNAseq and SMRTseq, this number includes spliced and unspliced reads. Because short reads are only useful for transcript characterization when they span a splice junction, the counts for short-reads represent the number of splice-junctionspanning reads.

B. The cumulative percentage of transcripts in each number of transcript classes, by strand. The $\mathrm{X}$-axis indicated the total number of transcript classes. The $\mathrm{Y}$ axis indicates the cumulative percentage of transcripts within those transcript classes. These plots indicate that most transcripts in most samples are contained within the first few transcript classes. transcripts for dRNAseq and SMRTseq data from SV40 (C), BKPyV Dunlop (D), and MPyV (E). The $X$ axis indicates the length of a read's alignment, while the $Y$ axis indicates the density/percentage of transcripts with a given alignment length. This plot shows that dRNAseq and SMRTseq data sample from RNA populations of

1094 Figure S2 - Transcript start sites and polyA tail lengths. different length. SV40 (left column), BKPyV Dunlop (middle column), and MPyV (right column). The arrows indicate the viral ORF positions. 
C. The distribution of polyA tail lengths for the 30-adenine ENO2 control (black), host (red), and viral (yellow) transcripts for SV40, BKPyV Dunlop, and MPyV. The $\mathrm{X}$ axis indicates the length of the polyA tail, while the $\mathrm{Y}$ axis indicates the density/percentage of transcripts with each length.

D. Ribosome occupancy of host transcripts in SV40-infected cells. Each grey dot is a host transcript. The red, blue, and black dots are specifically noted host transcripts. Ribosome occupancy is on the $\mathrm{Y}$ axis, while the $\mathrm{X}$ axis does not hold value. Lines on the violin plot indicate $1^{\text {st }}, 2^{\text {nd }}$, and $3^{\text {rd }}$ quartiles.

Figure S3 - SV40 transcriptome atlas, watch plots

A-C. Watch plots indicating all identified transcripts in SV40. (A) and (B) show transcripts that were identified in both dRNAseq and SMRTseq data, while (C) shows transcripts identified in SMRTseq only.

E. Barplots that show the abundance of each transcript type in the dRNAseq and SMRTseq data. Here, there are two dRNAseq bars (one per replicate). The $Y$ axis indicates the percentage of transcripts of the same strand. As discussed in the methods, alignment of superT and superT* was challenging, so the actual abundance of these transcripts is higher than reported here.

1117 Figure S4 - BKPyV Dunlop transcriptome atlas, watch plots 
A-C. Watch plots indicating all identified transcripts in BKPyV Dunlop. (A) and

(B) show transcripts that were identified in both dRNAseq and SMRTseq data, while (C) shows transcripts identified in dRNAseq only.

F. Barplots that show the abundance of each transcript type in the dRNAseq and SMRTseq data. The $Y$ axis indicates the percentage of transcripts of the same strand. As discussed in the methods, alignment of superT and superT* was challenging, so the actual abundance of these transcripts is higher than reported here.

Figure S5 - SV40 transcriptome atlas, late transcript read pileups and SMRTseq $(B)$. The arrows at the top of the plot indicate the viral ORF positions. Below the $\mathrm{X}$ axis, each row is an individual transcript, with exons indicated in red and splice junctions/introns indicated in pink. Above the $X$ axis

Figure S6 - SV40 transcriptome atlas, early transcript read pileups are histograms indicating the transcript start (blue) and transcript end (red) sites. (U: unspliced). and SMRTseq $(B)$. The arrows at the top of the plot indicate the viral ORF positions. Below the $\mathrm{X}$ axis, each row is an individual transcript, with exons 
indicated in red and splice junctions/introns indicated in pink. Above the $X$ axis are histograms indicating the transcript start (blue) and transcript end (red) sites. (U: unspliced).

Figure S7 - BKPyV Dunlop transcriptome atlas, late transcript read pileups

A, B. Read pileups showing the late transcripts identified in BKPyV Dunlop dRNAseq (A) and SMRTseq (B). The arrows at the top of the plot indicate the viral ORF positions. Below the $\mathrm{X}$ axis, each row is an individual transcript, with

Figure S8 - BKPyV Dunlop transcriptome atlas, early transcript read pileups exons indicated in red and splice junctions/introns indicated in pink. Above the $\mathrm{X}$ dRNAseq (A) and SMRTseq (B). The arrows at the top of the plot indicate the viral ORF positions. Below the $\mathrm{X}$ axis, each row is an individual transcript, with exons indicated in red and splice junctions/introns indicated in pink. Above the $X$ axis are histograms indicating the transcript start (blue) and transcript end (red) sites. (U: unspliced).

Figure S9 - Intron plots for all datasets studied 
A. Intron plots generated from short-read RNAseq. The arrows at the top and bottom of each panel indicate the position of viral ORFs. The lines indicate specific introns identified in the RNAseq data, with the 5' end on the top and the 3' end on the bottom. The blue color indicates late transcripts, with red indicating early transcripts. The size of the circles above and below the viral ORF maps indicate the percentage of junction-spanning reads with a 5' end (on top) or 3' end (on bottom) at that position. Junctions are plotted if they are at least $1 \%$ of early or late transcripts, except for the SV40 pA superT junction (transcript class 3) which is just below threshold but is of interest.

B. Another representation of intron plots for each virus. The top arrows indicate the position of viral ORFs. The $\mathrm{X}$ axis indicates the genomic position for each splice. The $\mathrm{Y}$ axis indicates a single transcript class, with that class' intron plotted as a

1177 Figure S10 - Alternative polyadenylation of early transcripts in SV40, BKPyV, and 1178 MPyV. Dunlop (B), and MPyV (C). The focus of these plots is the distribution of transcript end positions, which are the inner red arms. The region of APA of highlighted in blue, with the canonical transcript end sites highlighted in red. 
D. A cumulative incidence plot of transcript termination in SV40 (blue), BKPyV Dunlop (red), and MPyV (green). The X axis indicates the distance to the canonical polyA site, while the $Y$ axis indicates the percentage of transcripts that have terminated by that position.

E-G. Density plots showing the distribution of polyA tail lengths for LT and ST transcripts that end at the canonical site (solid) or undergo APA (dashed) for SV40 (E), BKPyV Dunlop (F), and MPyV (G). The $x$ axis indicates the length of the polyA tail, while the $Y$ axis indicates the density/proportion of transcripts with the given length.

Figure S11 - short-RNAseq (polyA) analysis of BKPyV Dik WT, M1, and M2

A-C. Intron plots generated from short-read (polyA) RNAseq of cells infected with

BKPyV WT, or the M1 or M2 mutants. The arrows at the top and bottom of each panel indicate the position of viral ORFs relative to the standard BKPyV genome - note that

1197 the genomes of mutants M1 and M2 are altered as indicated in Figure 5E. The lines

1198 indicate specific introns identified in the RNAseq data, with the 5' end on the top and the

11993 ' end on the bottom. The size of the circles above and below the viral ORF maps

1200 indicate the percentage of junction-spanning reads with a 5' end (on top) or 3' end (on

1201 bottom) at that position. Only early junctions that are at least $1 \%$ of early early

1202 transcripts are plotted. The superT junction is colored in gold. (A) Intron plot for BKPyV

1203 Dik WT. (B) Intron plot for BKPyV Dik M1. (C) Intron plot for BKPyV Dik M2. 
1205 Figure $\mathbf{S 1 2}$ - superT in MCPyV-associated MCC

1206 A. Sanger sequencing of an RT-PCR product from MCC J45_440, showing the 1207 superT-specific junction.

B. A schematic detailing the MCC $285 \mathrm{MCPyV}$ integration site, showing how it is possible that superT is generated via cis-splicing.

C. The assembled viral block in MCC tumor J45_440. This integration site is based on de-novo assembly using short whole genome sequencing reads. Despite only assembling one viral block, we found that 1) there are likely 2 copies of the viral genome, and 2) the 5' viral integration site appears to fall on chromosome 7 "after" the 3' viral integration site, observations consistent with the existence of two copies of the viral genome in tandem separated by a small segment of host DNA at this integration site. two copies of the early region. J17_296, and J11_285. The gene-map below the figure indicates the position of viral ORFs. Each lollipop is colored according to the nucleotide substitution identified. 
1225 Abend, J.R., Joseph, A.E., Das, D., Campbell-Cecen, D.B., and Imperiale, M.J. (2009).

1226 A truncated T antigen expressed from an alternatively spliced BK virus early mRNA.

1227 The Journal of general virology 90, 1238.

1228 Abere, B., Zhou, H., Li, J., Cao, S., Toptan, T., Grundhoff, A., Fischer, N., Moore, P.S.,

1229 and Chang, Y. (2020). Merkel Cell Polyomavirus Encodes Circular RNAs (circRNAs)

1230 Enabling a Dynamic circRNA/microRNA/mRNA Regulatory Network. Mbio 11, e03059-

123103020.

1232 Adami, G., Marlor, C., Barrett, N., and Carmichael, G.G. (1989). Leader-to-leader

1233 splicing is required for efficient production and accumulation of polyomavirus late

1234 mRNAs. Journal of virology 63, 85-93.

1235 Assetta, B., De Cecco, M., O’Hara, B., and Atwood, W.J. (2016). JC polyomavirus

1236 infection of primary human renal epithelial cells is controlled by a type I IFN-induced

1237 response. MBio 7, e00903-00916.

1238 Balázs, Z., Tombácz, D., Szücs, A., Snyder, M., and Boldogkői, Z. (2017). Long-read

1239 sequencing of the human cytomegalovirus transcriptome with the Pacific Biosciences

1240 RSII platform. Scientific data 4, 1-7.

1241 Carter, J.J., Daugherty, M.D., Qi, X., Bheda-Malge, A., Wipf, G.C., Robinson, K.,

1242 Roman, A., Malik, H.S., and Galloway, D.A. (2013). Identification of an overprinting

1243 gene in Merkel cell polyomavirus provides evolutionary insight into the birth of viral

1244 genes. Proceedings of the National Academy of Sciences 110, 12744-12749. 
1245 Depledge, D.P., Srinivas, K.P., Sadaoka, T., Bready, D., Mori, Y., Placantonakis, D.G.,

1246 Mohr, I., and Wilson, A.C. (2019). Direct RNA sequencing on nanopore arrays redefines

1247 the transcriptional complexity of a viral pathogen. Nature communications 10, 1-13.

1248 Dobin, A., Davis, C.A., Schlesinger, F., Drenkow, J., Zaleski, C., Jha, S., Batut, P.,

1249 Chaisson, M., and Gingeras, T.R. (2013). STAR: ultrafast universal RNA-seq aligner.

1250 Bioinformatics 29, 15-21.

1251 Eul, J., and Patzel, V. (2013). Homologous SV40 RNA trans-splicing: a new mechanism

1252 for diversification of viral sequences and phenotypes. RNA biology 10, 1689-1699.

1253 Freund, R., Sotnikov, A., Bronson, R.T., and Benjamin, T.L. (1992). Polyoma virus

1254 middle $\mathrm{T}$ is essential for virus replication and persistence as well as for tumor induction

1255 in mice. Virology 191, 716-723.

1256 Garalde, D.R., Snell, E.A., Jachimowicz, D., Sipos, B., Lloyd, J.H., Bruce, M., Pantic, N.,

1257 Admassu, T., James, P., and Warland, A. (2018). Highly parallel direct RNA sequencing

1258 on an array of nanopores. Nature methods 15, 201-206.

1259 Garren, S.B., Kondaveeti, Y., Duff, M.O., and Carmichael, G.G. (2015). Global analysis

1260 of mouse polyomavirus infection reveals dynamic regulation of viral and host gene

1261 expression and promiscuous viral RNA editing. PLoS pathogens 11, e1005166.

1262 Ghosh, P., Reddy, V., Swinscoe, J., Lebowitz, P., and Weissman, S. (1978).

1263 Heterogeneity and 5'-terminal structures of the late RNAs of simian virus 40. Journal of 1264 molecular biology 126, 813-846. 
1265 Good, P.J., Welch, R.C., Ryu, W.-S., and Mertz, J.E. (1988). The late spliced 19S and

1266 16S RNAs of simian virus 40 can be synthesized from a common pool of transcripts.

1267 Journal of virology 62, 563-571.

1268 Gottlieb, K.A., and Villarreal, L.P. (2001). Natural biology of polyomavirus middle T

1269 antigen. Microbiology and molecular biology reviews 65, 288-318.

1270 Jiang, M., Abend, J.R., Johnson, S.F., and Imperiale, M.J. (2009a). The role of

1271 polyomaviruses in human disease. Virology 384, 266-273.

1272 Jiang, M., Abend, J.R., Tsai, B., and Imperiale, M.J. (2009b). Early events during BK

1273 virus entry and disassembly. Journal of virology 83, 1350-1358.

1274 Johnson, M., Zaretskaya, I., Raytselis, Y., Merezhuk, Y., McGinnis, S., and Madden,

1275 T.L. (2008). NCBI BLAST: a better web interface. Nucleic acids research 36, W5-W9.

1276 Kamen, R., Favaloro, J., and Parker, J. (1980a). Topography of the three late mRNA's

1277 of polyoma virus which encode the virion proteins. Journal of virology 33, 637-651.

1278 Kamen, R., Favaloro, J., Parker, J., Treisman, R., Lania, L., Fried, M., and Mellor, A.

1279 (1980b). Comparison of polyoma virus transcription in productively infected mouse cells

1280 and transformed rodent cell lines. Paper presented at: Cold Spring Harbor symposia on

1281 quantitative biology (Cold Spring Harbor Laboratory Press).

1282 Keller, M.W., Rambo-Martin, B.L., Wilson, M.M., Ridenour, C.A., Shepard, S.S., Stark,

1283 T.J., Neuhaus, E.B., Dugan, V.G., Wentworth, D.E., and Barnes, J.R. (2018). Direct

1284 RNA sequencing of the coding complete influenza A virus genome. Scientific reports 8 , 1285 1-8. 
1286 Kim, D., Lee, J.-Y., Yang, J.-S., Kim, J.W., Kim, V.N., and Chang, H. (2020). The

1287 architecture of SARS-CoV-2 transcriptome. Cell 181, 914-921. e910.

1288 Kress, M., May, E., Cassingena, R., and May, P. (1979). Simian virus 40-transformed

1289 cells express new species of proteins precipitable by anti-simian virus 40 tumor serum.

1290 Journal of virology 31, 472-483.

1291 Krueger, F. (2016). Babraham Bioinformatics-Trim Galore.

1292 Lee, S., Micalizzi, D., Truesdell, S.S., Bukhari, S.I., Boukhali, M., Lombardi-Story, J.,

1293 Kato, Y., Choo, M.-K., Dey-Guha, I., and Ji, F. (2020). A post-transcriptional program of

1294 chemoresistance by AU-rich elements and TTP in quiescent leukemic cells. Genome

1295 biology $21,1-23$.

1296 Legon, S., Flavell, A.J., Cowie, A., and Kamen, R. (1979). Amplification in the leader

1297 sequence of late polyoma virus mRNAs. Cell 16, 373-388.

1298 Li, H. (2018). Minimap2: pairwise alignment for nucleotide sequences. Bioinformatics

$129934,3094-3100$.

1300 Loman, N.J., Quick, J., and Simpson, J.T. (2015). A complete bacterial genome

1301 assembled de novo using only nanopore sequencing data. Nature methods 12, 733-

1302735.

1303 Luo, Y., and Carmichael, G.G. (1991). Splice site skipping in polyomavirus late pre-

1304 mRNA processing. Journal of virology 65, 6637-6644.

1305 Nguyen, K.D., Lee, E.E., Yue, Y., Stork, J., Pock, L., North, J.P., Vandergriff, T., 1306 Cockerell, C., Hosler, G.A., and Pastrana, D.V. (2017). Human polyomavirus 6 and 7 
1307 are associated with pruritic and dyskeratotic dermatoses. Journal of the American

1308 Academy of Dermatology 76, 932-940. e933.

1309 Nomburg, J., Meyerson, M., and DeCaprio, J.A. (2020). Pervasive generation of non1310 canonical subgenomic RNAs by SARS-CoV-2. Genome medicine 12, 1-14.

1311 Norbury, C.J., and Fried, M. (1987). Polyomavirus early region alternative poly (A) site:

1312 3'-end heterogeneity and altered splicing pattern. Journal of virology 61, 3754-3758.

1313 Price, A.M., Hayer, K.E., Mclntyre, A.B., Gokhale, N.S., Abebe, J.S., Della Fera, A.N.,

1314 Mason, C.E., Horner, S.M., Wilson, A.C., and Depledge, D.P. (2020). Direct RNA

1315 sequencing reveals m 6 A modifications on adenovirus RNA are necessary for efficient 1316 splicing. Nature communications 11, 1-17.

1317 Quinlan, A.R., and Hall, I.M. (2010). BEDTools: a flexible suite of utilities for comparing 1318 genomic features. Bioinformatics 26, 841-842.

1319 Reddy, V.B., Ghosh, P.K., Lebowitz, P., and Sherman M, W. (1978). Gaps and

1320 duplicated sequences in the leaders of SV40 16S RNA. Nucleic acids research 5, 419513214214.

1322 Riley, M.I., Yoo, W., Mda, N.Y., and Folk, W.R. (1997). Tiny T antigen: an autonomous 1323 polyomavirus T antigen amino-terminal domain. Journal of virology 71, 6068-6074.

1324 Rosenstein, R.K., Pastrana, D.V., Starrett, G.J., Sapio, M.R., Hill, N.T., Jo, J.-H., Lee, 1325 C.-C.R., ladarola, M.J., Buck, C.B., and Kong, H.H. (2021). Host-Pathogen Interactions 1326 in Human Polyomavirus 7-Associated Pruritic Skin Eruption. The Journal of 1327 investigative dermatology 141, 1344-1348. e1348. 
1328 Saribas, A.S., DeVoto, J., Golla, A., Wollebo, H.S., White, M.K., and Safak, M. (2018).

1329 Discovery and characterization of novel trans-spliced products of human polyoma JC

1330 virus late transcripts from PML patients. Journal of cellular physiology 233, 4137-4155.

1331 Schmidlin, K., Kraberger, S., Cook, C., DeNardo, D.F., Fontenele, R.S., Van Doorslaer,

1332 K., Martin, D.P., Buck, C.B., and Varsani, A. (2021). A novel lineage of polyomaviruses

1333 identified in bark scorpions. bioRxiv.

1334 Seif, I., Khoury, G., and Dhar, R. (1979). The genome of human papovavirus BKV. Cell $133518,963-977$.

1336 Shuda, M., Feng, H., Kwun, H.J., Rosen, S.T., Gjoerup, O., Moore, P.S., and Chang, Y.

1337 (2008). T antigen mutations are a human tumor-specific signature for Merkel cell

1338 polyomavirus. Proceedings of the National Academy of Sciences 105, 16272-16277.

1339 Smith, A.E., Smith, R., and Paucha, E. (1979). Characterization of different tumor

1340 antigens present in cells transformed by simian virus 40 . Cell 18, 335-346.

1341 Starrett, G.J., Thakuria, M., Chen, T., Marcelus, C., Cheng, J., Nomburg, J., Thorner,

1342 A.R., Slevin, M.K., Powers, W., and Burns, R.T. (2020). Clinical and molecular

1343 characterization of virus-positive and virus-negative Merkel cell carcinoma. Genome

1344 medicine 12, 1-22.

1345 Starrett, G.J., Yu, K., Golubeva, Y., Lenz, P., Piaskowski, M.L., Peterson, D., Dean, M., 1346 Israni, A., Hernandez, B.Y., Tucker, T.C., et al. (2021). Common Mechanisms of Virus-

1347 Mediated Oncogenesis in Bladder Cancers Arising In Solid Organ Transplant

1348 Recipients. medRxiv, 2021.2011.2011.21266080. 
1349 Theiss, J.M., Günther, T., Alawi, M., Neumann, F., Tessmer, U., Fischer, N., and

1350 Grundhoff, A. (2015). A comprehensive analysis of replicating Merkel cell polyomavirus

1351 genomes delineates the viral transcription program and suggests a role for mcv-miR-M1

1352 in episomal persistence. PLoS pathogens 11, e1004974.

1353 Treisman, R. (1980). Characterisation of polyoma late mRNA leader sequences by

1354 molecular cloning and DNA sequence analysis. Nucleic acids research 8, 4867-4888.

1355 Tremblay, J.D., Sachsenmeier, K.F., and Pipas, J.M. (2001). Propagation of wild-type

1356 and mutant SV40. In SV40 Protocols (Springer), pp. 1-7.

1357 Trowbridge, P.W., and Frisque, R.J. (1995). Identification of three new JC virus proteins

1358 generated by alternative splicing of the early viral mRNA. Journal of neurovirology 1 ,

1359 195-206.

1360 Yang, R., Lee, E.E., Kim, J., Choi, J.H., Kolitz, E., Chen, Y., Crewe, C., Salisbury, N.J.,

1361 Scherer, P.E., and Cockerell, C. (2021). Characterization of ALTO-encoding circular

1362 RNAs expressed by Merkel cell polyomavirus and trichodysplasia spinulosa

1363 polyomavirus. PLoS pathogens 17, e1009582.

1364 Zerrahn, J., Knippschild, U., Winkler, T., and Deppert, W. (1993). Independent

1365 expression of the transforming amino-terminal domain of SV40 large I antigen from an

1366 alternatively spliced third SV40 early mRNA. The EMBO journal 12, 4739-4746.

1367 Zhao, L., and Imperiale, M.J. (2019). Establishing Renal Proximal Tubule Epithelial-

1368 Derived Cell Lines Expressing Human Telomerase Reverse Transcriptase for Studying

1369 BK Polyomavirus. Microbiology resource announcements 8, e01129-01119. 
bioRxiv preprint doi: https://doi.org/10.1101/2021.11.12.468421; this version posted November 13,2021 . The copyright holder for this preprint (which was not certified by peer review) is the author/funder, who has granted bioRxiv a license to display the preprint in perpetuity. It is made available under aCC-BY-NC-ND 4.0 International license.

1370 Zou, W., Vue, G.S., Assetta, B., Manza, H., Atwood, W.J., and Imperiale, M.J. (2020).

1371 Control of archetype BK polyomavirus microRNA expression. Journal of Virology 95,

1372 e01589-01520.

1373

1374 
bioRxiv preprint doi: https://doi.org/10.1101/2021.11.12.468421; this version posted November 13, 2021. The copyright holder for this preprint (which was not certified by peer review) is the author/funder, who has granted bioRxiv a license to display the preprint in perpetuity. It is made Figure 1 available under aCC-BY-NC-ND 4.0 International license.

A

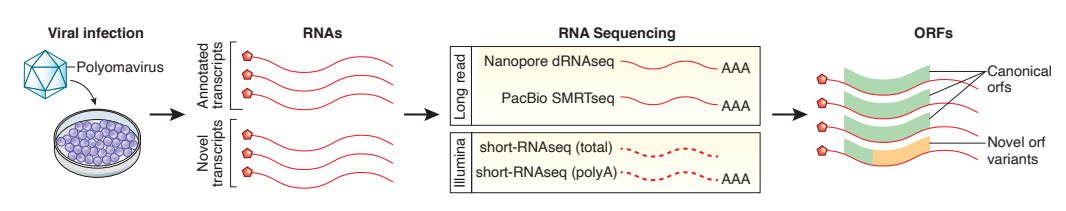

B Transcript processing strategy

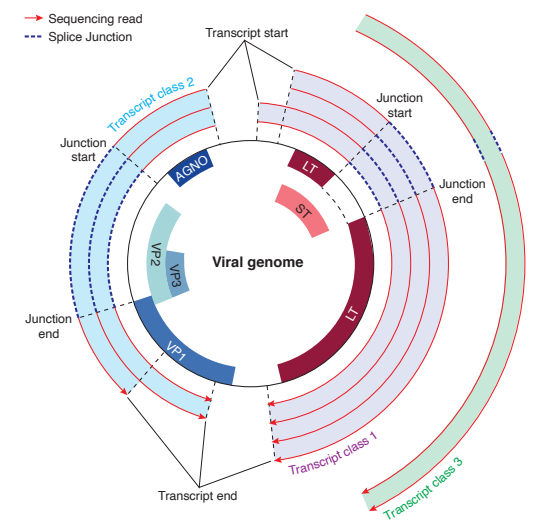

C

$\mathrm{F}$

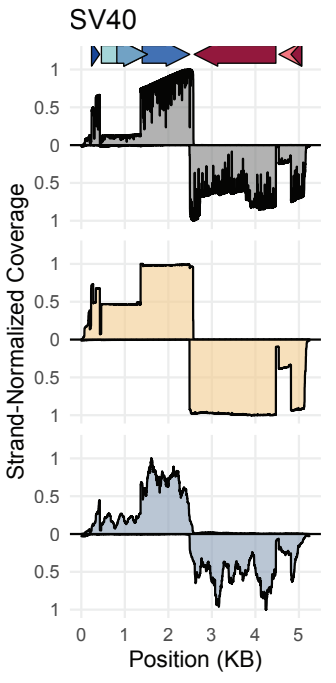

BKPyV
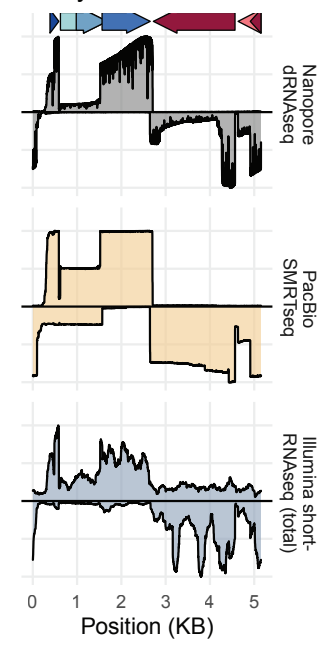

SV40

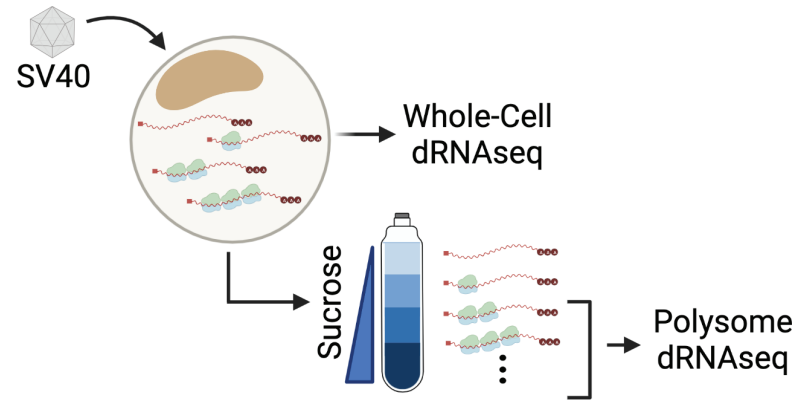

D

SV40

E

E BKPyV

G

\section{SV40 Whole-Cell vs. Polysome Transcript Abundance}

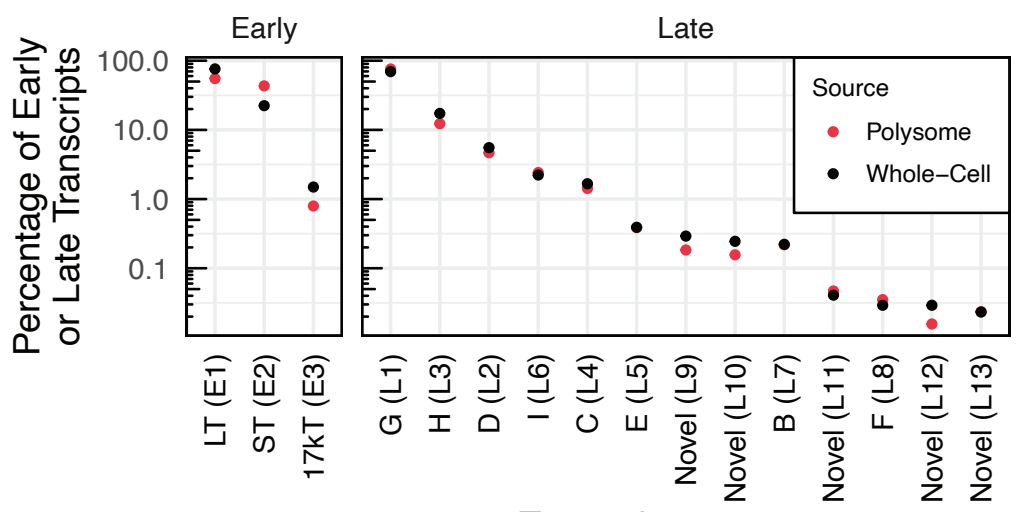

\section{Transcript}

Figure 1 - RNA sequencing expands known SV40 and BKPyV transcript diversity.

A. Overview of experimental procedures. Cells were infected with a polyomavirus, and RNAs extracted. RNA was sequenced using long-read (Nanopore dRNAseq and PacBio SMRTseq) and short-read (Illumina short-RNAseq (total) and short-RNAseq (polyA)). Transcripts were analyzed, and the impact of observed splice events on viral open reading frames was assessed.

B. Mechanism of transcript clustering in this study. Transcripts were aligned to the viral genome and grouped into transcript classes based on the presence of shared introns. Thus, within a transcript class there may be variation in the exact transcript start and end positions. This clustering strategy was used for both long- and short-RNAseq data. C. Viral RNA sequence coverage for SV40 and BKPyV as determined from dRNAseq, SMRTseq, and short-RNAseq (total) data. The Y axis indicates the scaled coverage, with X axis indicating the position on the viral genome. Coverage for late transcripts (mapping to the + strand) is above the $x$ axis, while coverage for early transcripts (mapping to the - strand) is below the $x$ axis. Coverage is scaled separately for each strand such that the maximum observed coverage for each strand is 1 . Arrows at the top of the plot indicate the positions of viral genes.

D-E. UpSet plot indicating the overlap between existing transcript annotations, dRNAseq data, and SMRTseq data for SV40 (D) and BKPyV Dunlop (E). Bars indicating overlap with existing transcript annotations are black, while those indicating no overlap with existing annotations are blue. These blue bars indicate the number of novel, unannotated transcripts identified.

F. Overview of polysome profiling of SV40-infected cells. BSC40 cells were infected with SV40. Cells were lysed, and a portion of the lysate was subjected to dRNAseq (representative of the RNA content of the whole cell). The remaining lysates was centrifuged through a sucrose gradient, after which fractions containing RNA associated with two or more ribosomes were pooled and subjected to dRNAseq.

G. Relative abundance of SV40 early and late transcripts in the whole-cell and polysome fractions of SV40-infected cells. Y-axis indicates the percentage of early or late transcripts and is $\log$ scale. $X$ axis indicates each transcript, with black dots indicating each transcript's whole-cell relative abundance and red dots indicating each transcript's polysome relative abundance. 
bioRxiv preprint doi: https://doi.org/10.1101/2021.11.12.468421; this version posted November 13,2021 . The copyright holder for this preprint (which was not certified by peer review) is the author/funder, who has granted bioRxiv a license to display the preprint in perpetuity. It is made Figure 2 available under aCC-BY-NC-ND 4.0 International license.

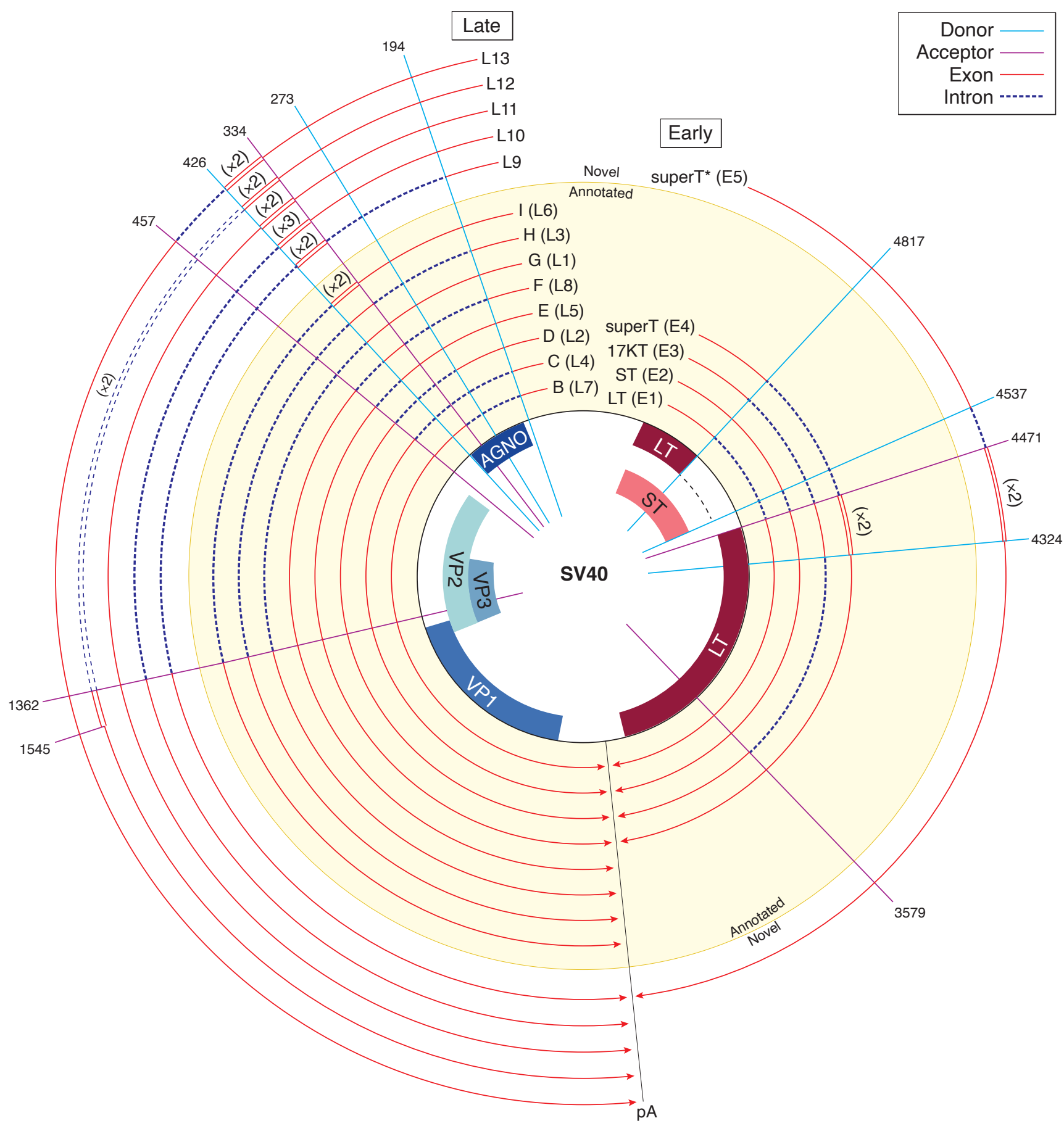

Figure 2 - Annotated and novel SV40 transcripts.

A. Transcripts are shown relative to the viral genome. Each line is a viral transcript, with red lines indicating exons and dashed blue lines indicating introns. Spokes indicate the positions of common splice donors and splice acceptors. Transcripts that were annotated prior to this study are on a yellow background, and novel transcripts are on a while background. Wraparound transcription that results in multiple copies of a region is annotated with double lines, and the number of copies

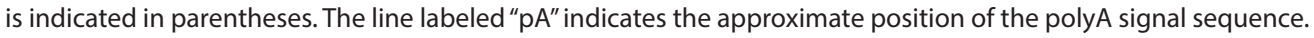


bioRxiv preprint doi: https://doi.org/10.1101/2021.11.12.468421; this version posted November 13,2021 . The copyright holder for this preprint (which was not certified by peer review) is the author/funder, who has granted bioRxiv a license to display the preprint in perpetuity. It is made Figure 3 available under aCC-BY-NC-ND 4.0 International license.

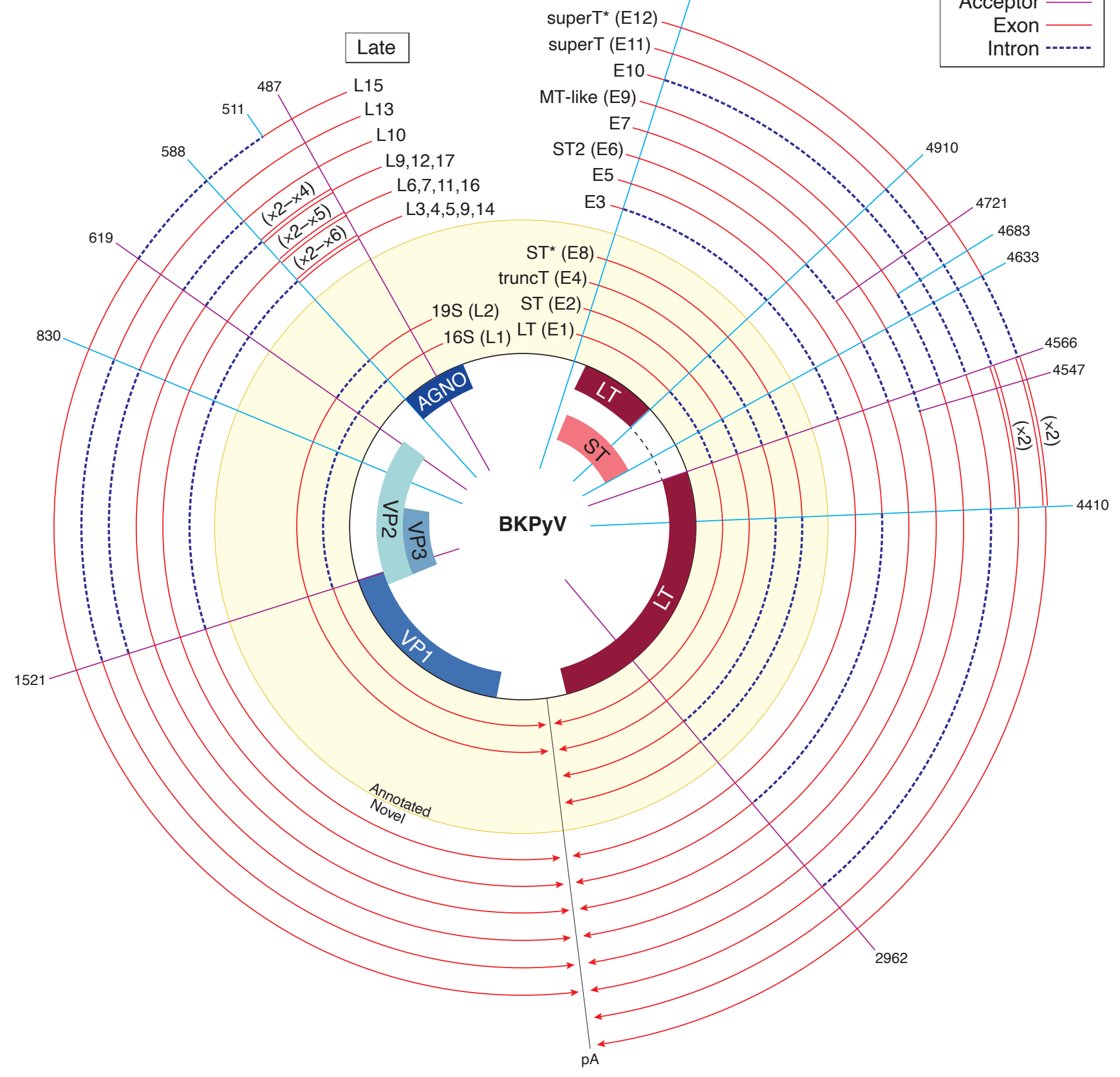

Figure 3 - Annotated and novel BKPyV transcripts.

A. Transcripts are shown relative to the viral genome. Each line is a viral transcript, with red lines indicating exons and dashed blue lines indicating introns. Spokes indicate the positions of common splice donors and splice acceptors. Transcripts that were annotated prior to this study are on a yellow background, and novel transcripts are on a while background. Wraparound transcription that results in multiple copies of a region is annotated with double lines, and the number of copies is indicated in parentheses. The line labeled " $\mathrm{pA}$ " indicates the approximate position of the polyA signal sequence. 
bioRxiv preprint doi: $h t t p s ; / / d o i . o r g / 10.1101 / 2021.11 .12 .468421$; this version posted November 13,2021 . The copyright holder for this preprint A (which was not certifified by'peer review) is the author/funder, who has granted bioRxiv a license to display the preprint in perpetuity. It is made

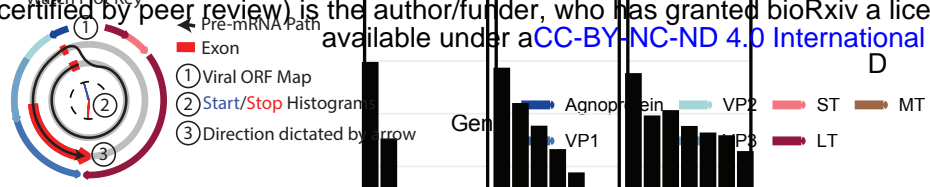

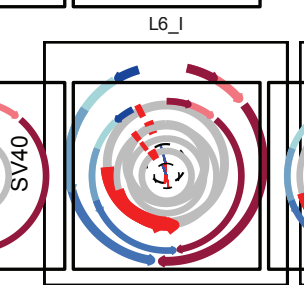

L3_Novel

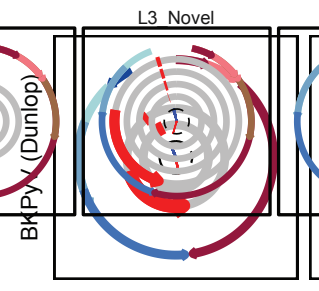

C
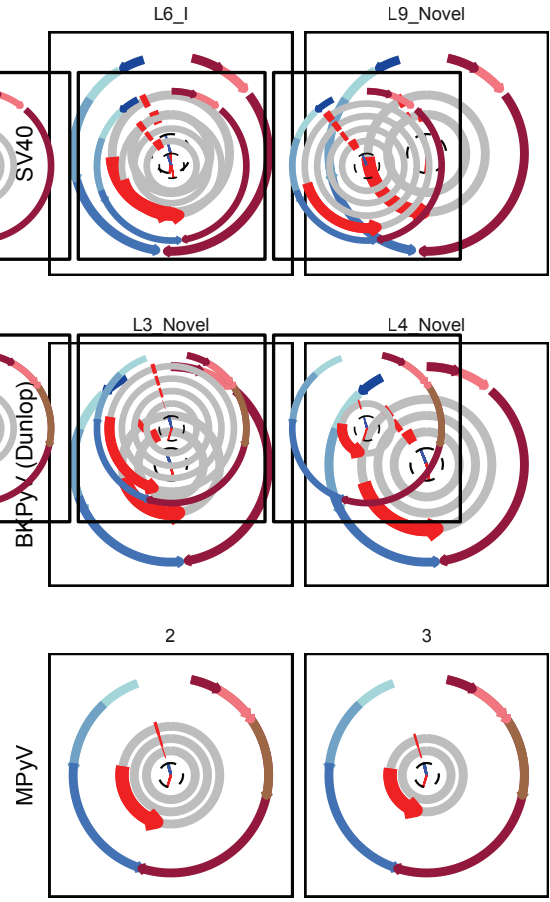

L4_Novel
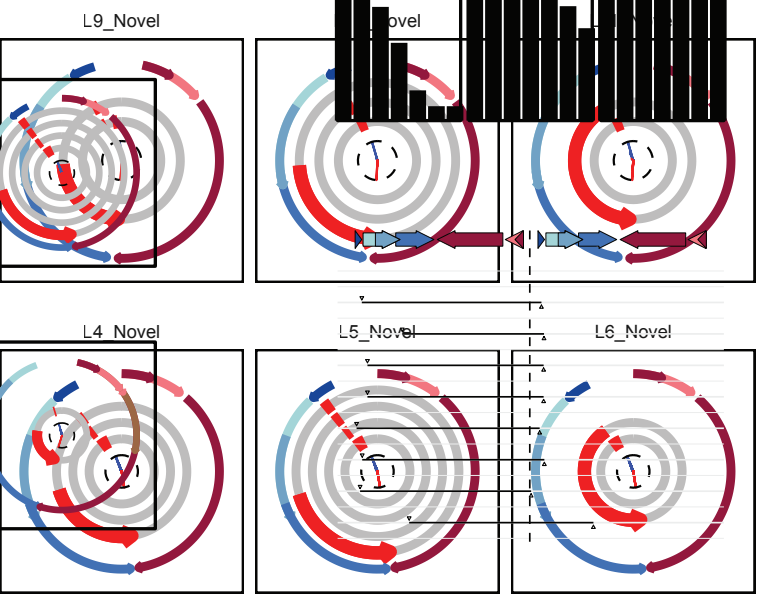

L5_Novel
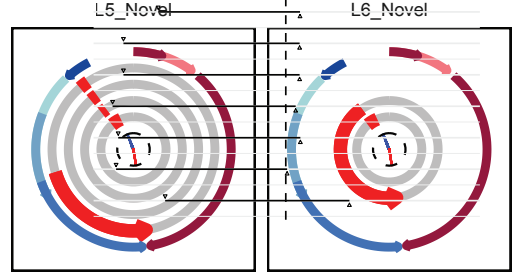

$\mathrm{F}$

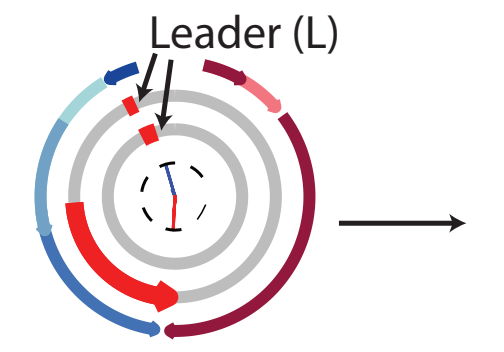

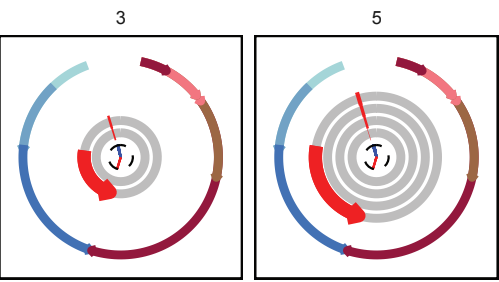

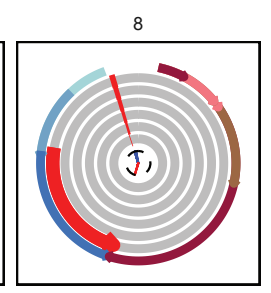

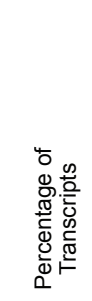

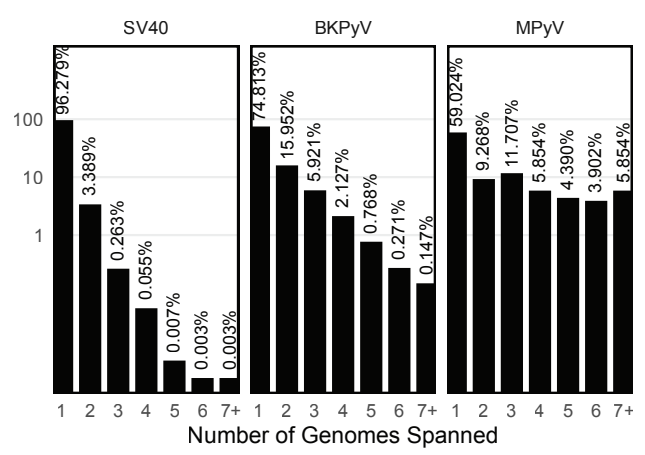

E

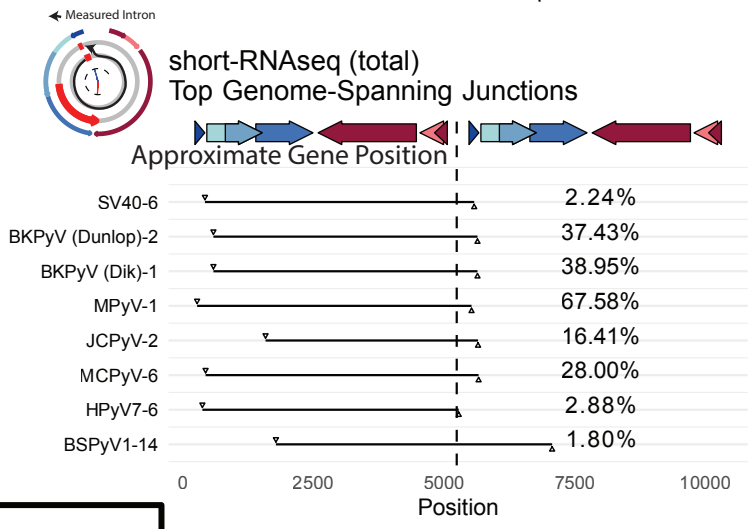

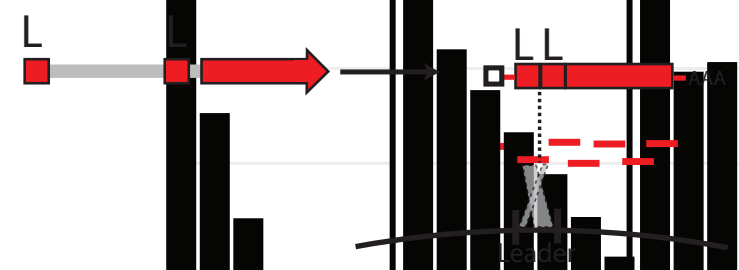

Transcript of origin

Short reads

Viral reference genome

Figure 4 - Pervasive wraparound transcription across PyV

A-C. Watch plots indicating the top 4 highest abundance late wraparound transcript classes in dRNAseq data from SV40 (A), BKPyV Dunlop (B), and MPyV (C). The outer ring of each watch plot indicates the position of the viral ORFs. The inner arms are histograms detailing the distribution of transcript starts (in blue) and ends (in red) for transcripts within each transcript class. The red segments indicate exons. Transcripts start in the innermost ring - a second or third ring indicates that the pre-mRNA that generated the transcript must have circled the viral genome multiple times. The $3^{\prime}$ end of the transcript and the direction in which these plots are oriented is indicated by the red arrow at the end of the last exon segment. The red exon segments start at the most common transcript start site within the transcript class, and end at the most common transcript end site within the class. The watch plot key shows an example of the path of the pre-mRNA for SV40 transcript class L6_I.

D. Bar plots indicating the percentage of late transcripts that span a given number of genome lengths in SV40, BKPyV Dunlop, and MPyV dRNAseq data.

E. The leader-leader junction, that connects the pre-mRNA from one genome to the subsequent wraparound, was identified in Illumina short-RNAseq (total) data. The intron in question is plotted as a black line in this plot, with the $\mathrm{x}$ axis indicating the genomic position of the intron. The top late wraparound transcript for each virus was plotted. The gene map indicates the approximate gene position and is accurate for SV40 - the exact position of the viral genes varies between viruses. Percentages indicate the percentage of late junction-spanning transcripts that support the plotted wraparound leader-leader junction.

F. Schematic illustrating how leader-leader wraparound transcription can be detected from short read short-RNAseq (total). Leader-leader splicing can be seen as a repetitive exon in watch plots from long-read RNAseq data. Ultimately, there was an original processed mRNA in the cell that contained two tandem leader sequences. When this transcript of origin is sequenced via short read sequencing, reads will be generated across its length. A minority of these reads will span the leader-leader junction, and mapping against the viral reference genome can be used to uncover leader-leader splicing. 
bioRxiv preprint doi: https://doi.org/10.1101/2021.11.12.468421; this version posted November 13, 2021. The copyright holder for this preprint Figuke b was not certified by peer review) is the author/funder, who has granted bioRxiv a license to display the preprint in perpetuity. It is made

A ST2

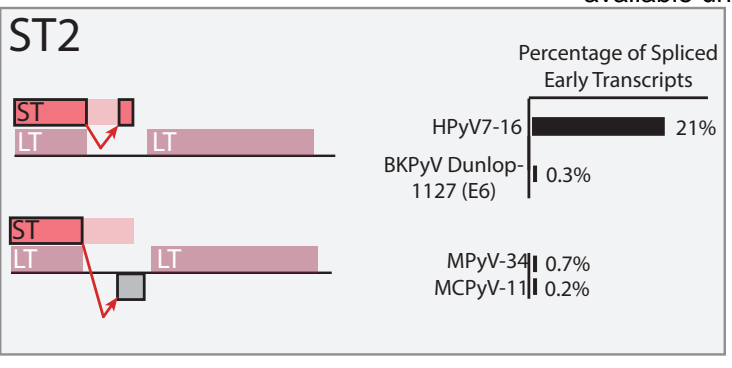
available undeß aCC-BY-NC-ND 4.0 International license.

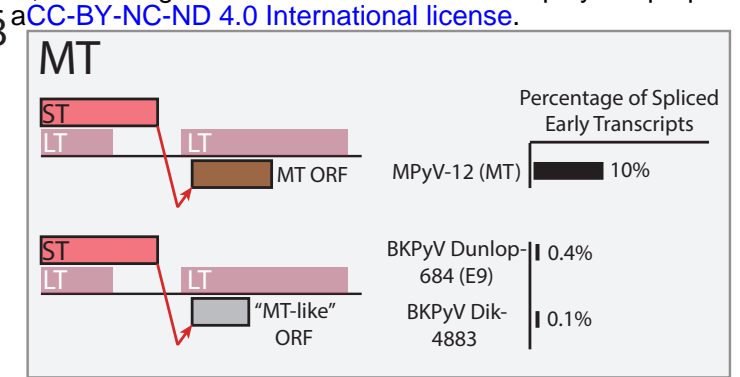

C
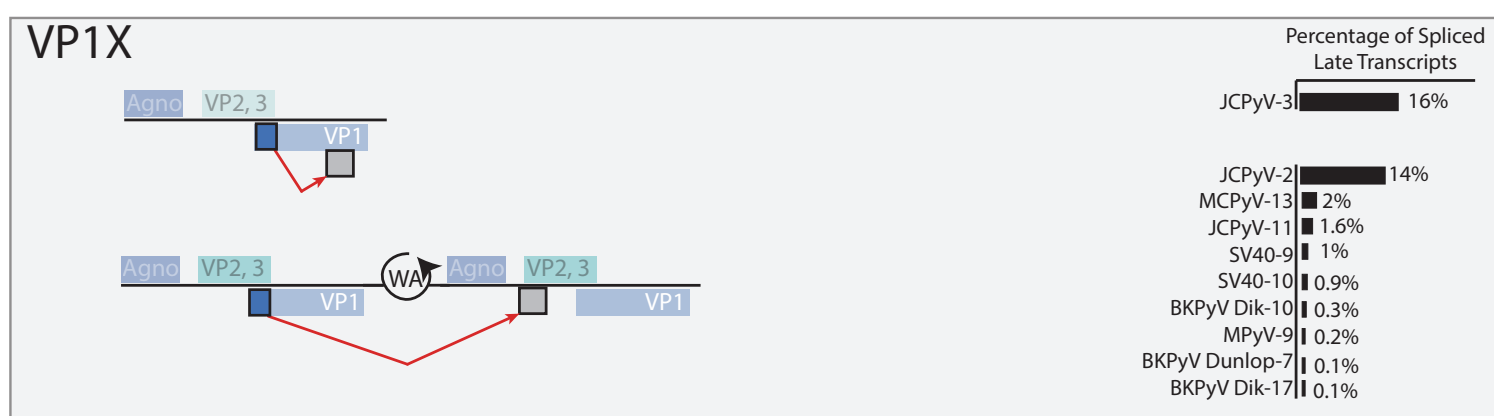

D

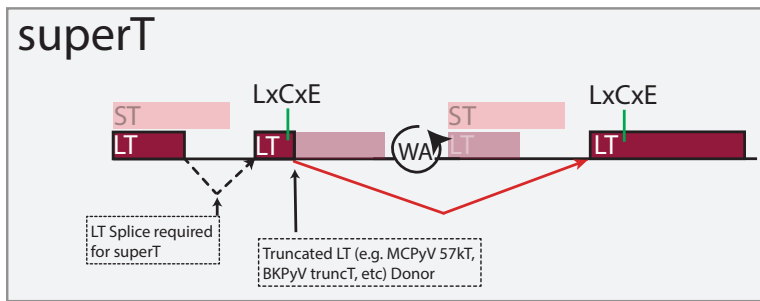

E
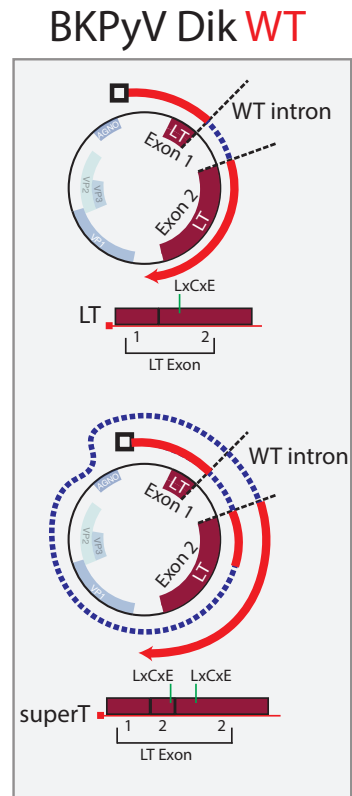

BKPyV Dik M1

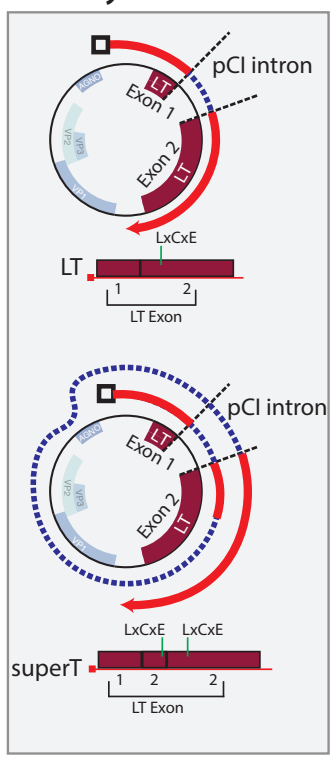

BKPyV Dik M2

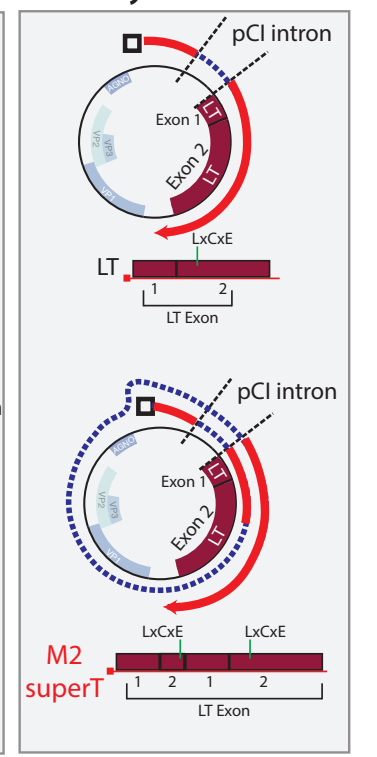

$\mathrm{F}$

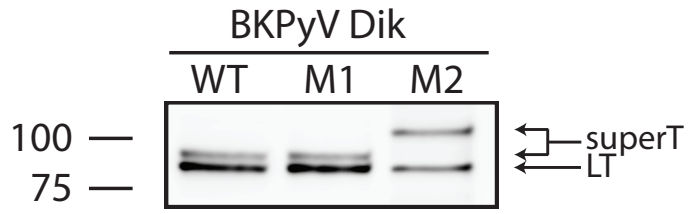

Figure 5 - Detection of novel, conserved splicing events that expand PyV coding capacity.

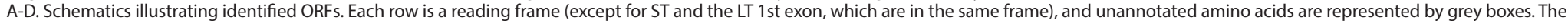

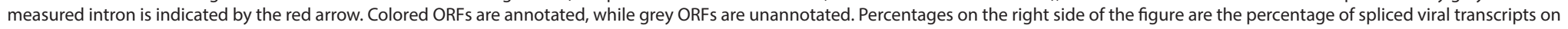

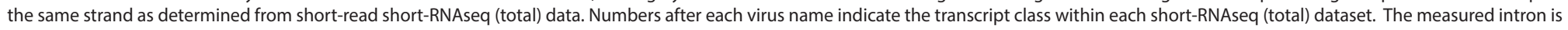
indicated by the red arrow.

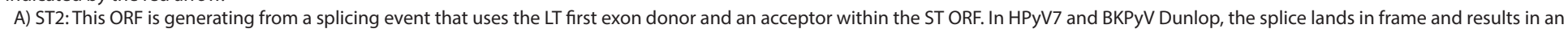
internal deletion within ST. In MPyV and MCPyV the splice lands out of frame, resulting in an ORF that contains the N-terminal region of ST and novel amino acids at the C terminus.

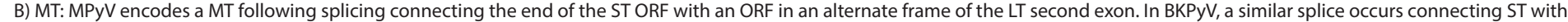
an MT-like ORF in an alternative frame of the LT second exon.

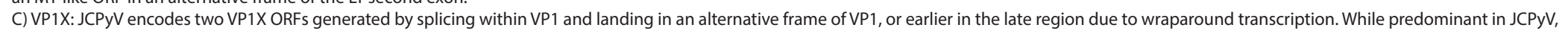
VP1X is likewise present in many other PyV.

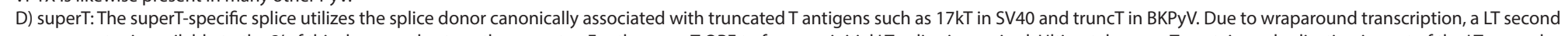

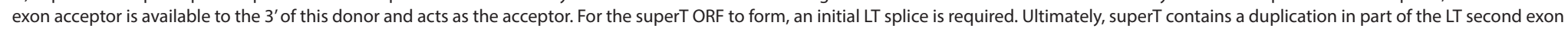
that includes the RB-binding LxCxE motif.

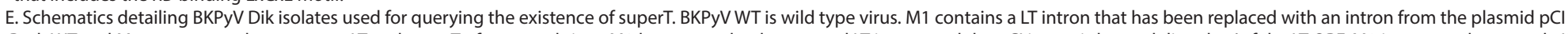

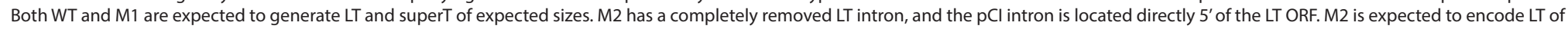
expected size, but a larger superT variant due to incorporation of a second copy of the LT first exon.

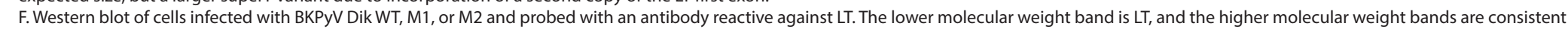
with superT. 
bioRxiv preprint doi: https://doi.org/10.1101/2021.11.12.468421; this version posted November 13,2021 . The copyright holder for this preprint (which was not certified by peer review) is the author/funder, who has granted bioRxiv a license to display the preprint in perpetuity. It is made Figure 6 available under aCC-BY-NC-ND 4.0 International license.

A

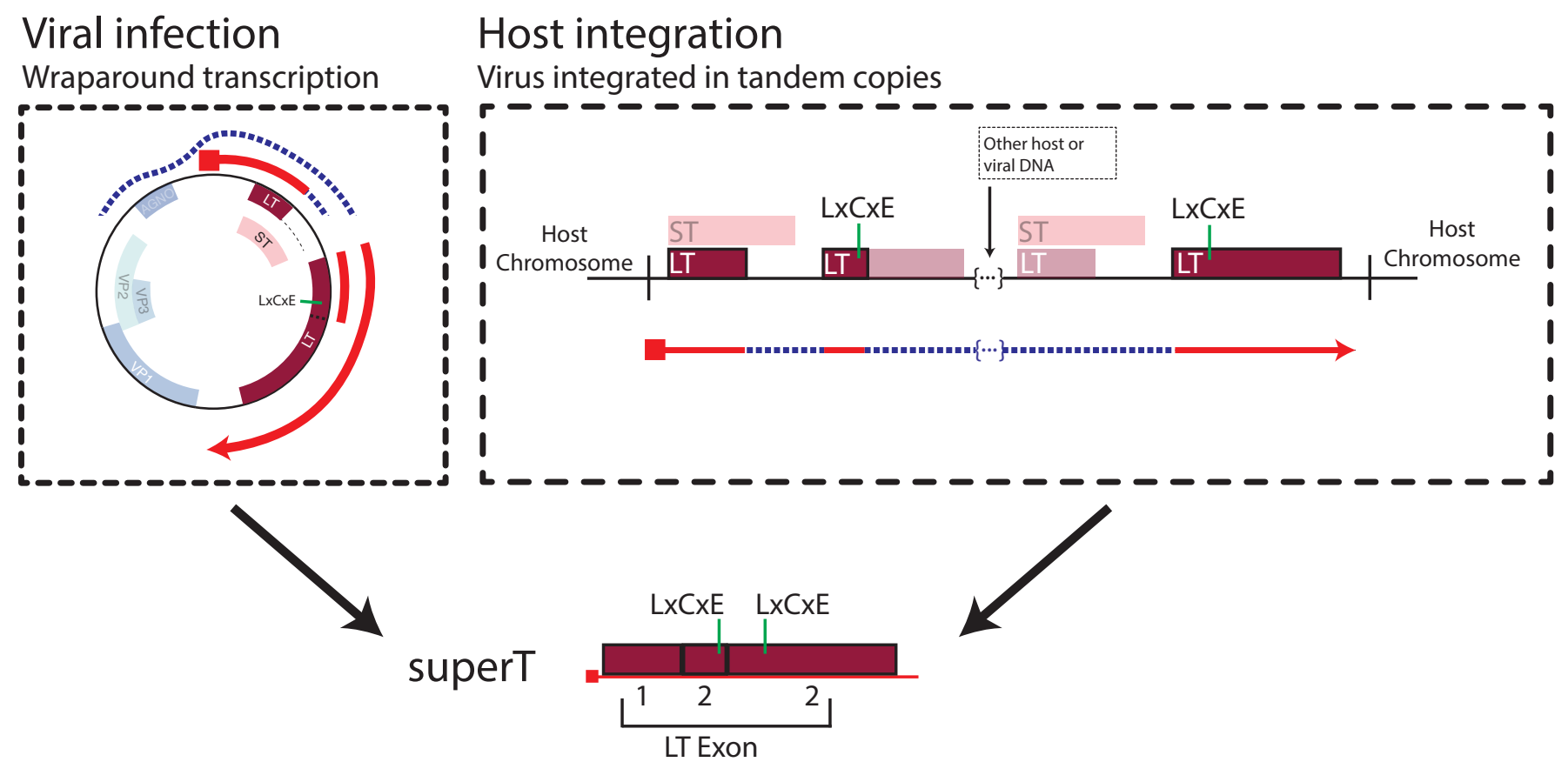

B

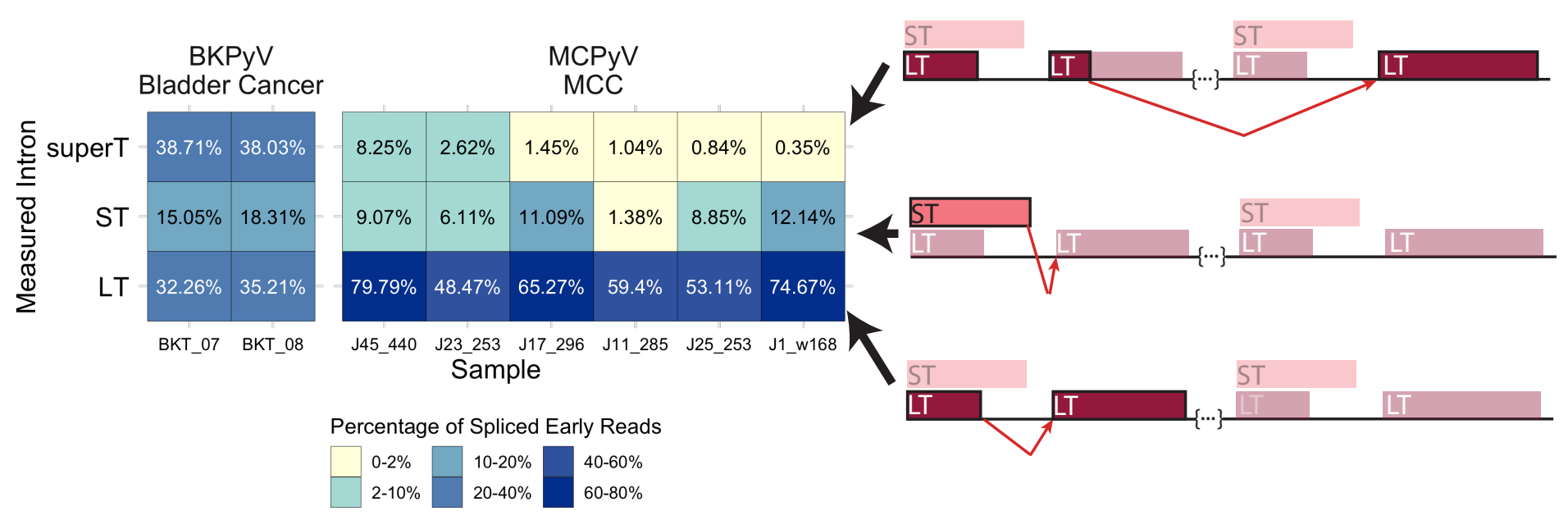

Figure 6 - Detection superT-encoding transcripts in PyV-associated cancers

A. Schematic detailing the generation of superT during lytic infection as compared to from integrated virus in cancer. During viral infection, the RNA polymerase can circle the viral genome multiple times, resulting in a pre-mRNA that can be spliced to generate superT. In the case of host integration, a polyomavirus can be integrated in tandem copies such that a pre-mRNA is generated with more than one copy of the viral early region. This pre-mRNA can be similarly spliced to generate a superT transcript. B. Heatmap indicating the abundance of the superT, ST, and LT introns from RNAseq data from two replicates of a BKPyV-positive bladder cancer and six MCPyV-associated MCCs. Percentages indicate the percentage of spliced early viral reads for each sample. The splice measured in each row is indicated by the red arrow in the schematics on the right side of the figure. 
bioRxiv preprint doi: https://doi.org/10.1101/2021.11.12.468421; this version posted November 13, 2021. The copyright holder for this preprint (which was not certified by peer review) is the author/funder, who has granted bioRxiv a license to display the preprint in perpetuity. It is made Fig S1 available under aCC-BY-NC-ND 4.0 International license.

A

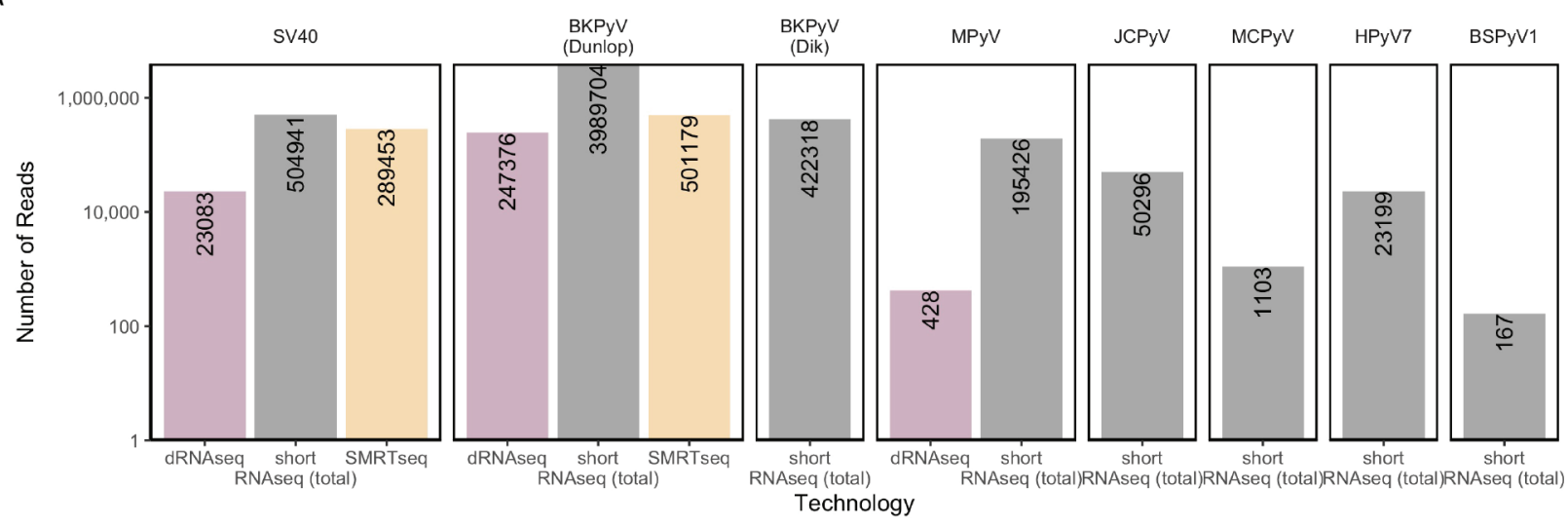

B

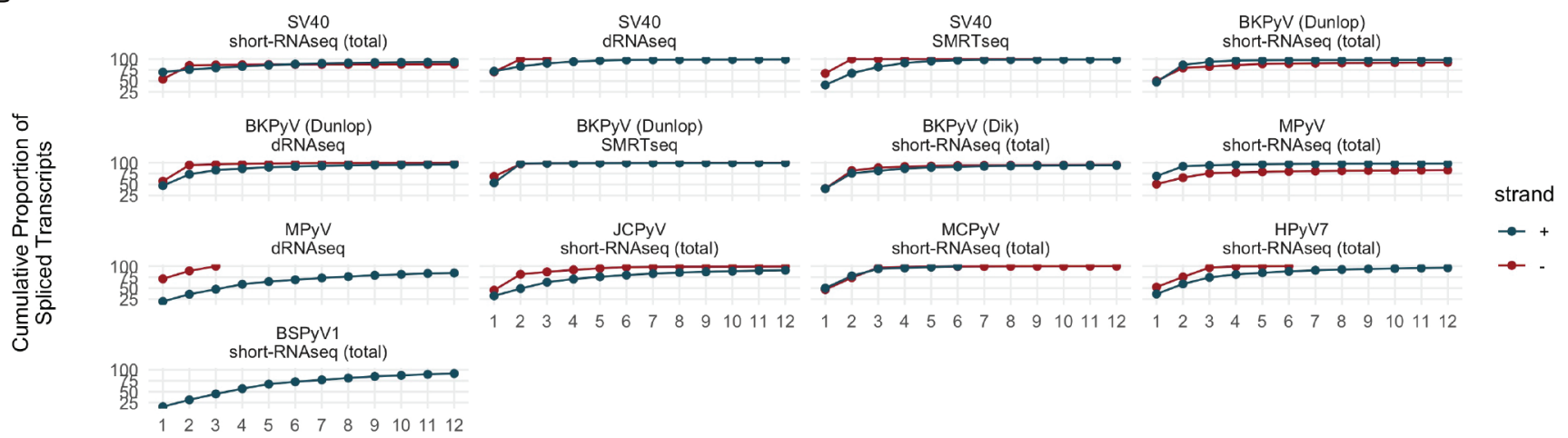

C

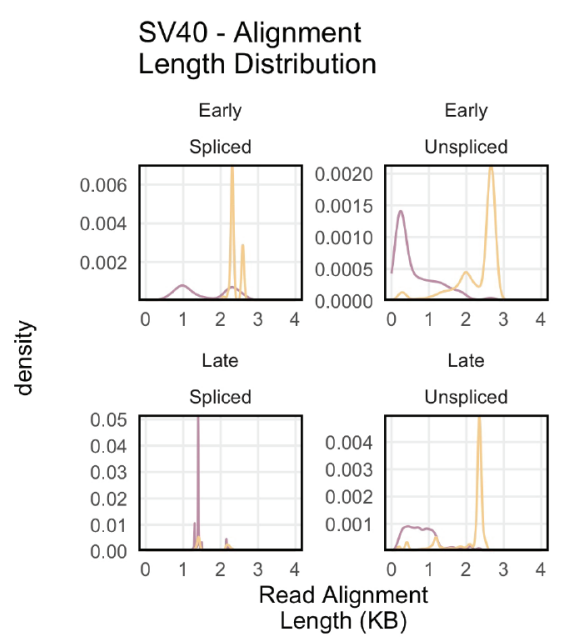

D

BKPyV (Dunlop) - Alignment Length Distribution

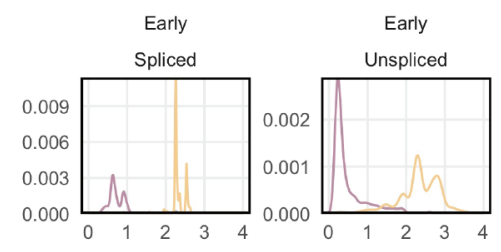

Late

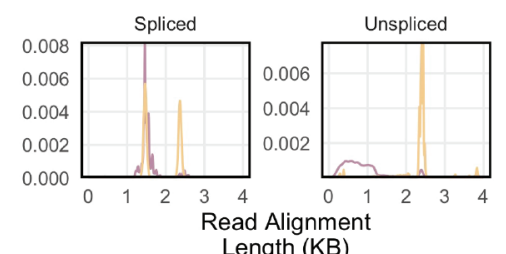

Length (KB)
$\mathrm{E}$

MPyV - Alignment Length Distribution

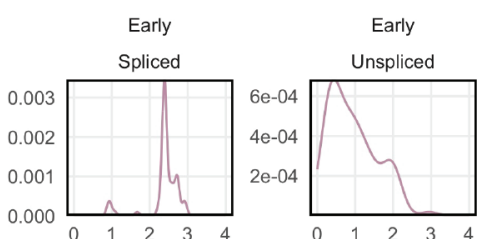

Technology

- dRNAseq

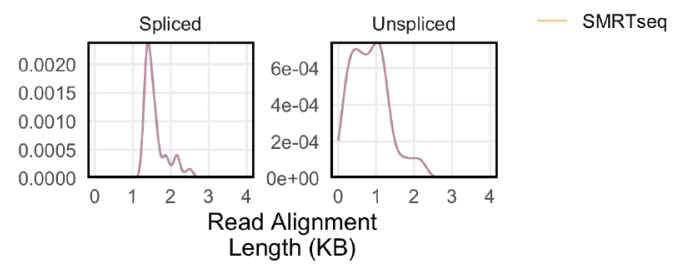

- SMRTseq 
bioRxiv preprint doi: https://doi.org/10.1101/2021.11.12.468421; this version posted November 13,2021 . The copyright holder for this preprint (which was not certified by peer review) is the author/funder, who has granted bioRxiv a license to display the preprint in perpetuity. It is made available under aCC-BY-NC-ND 4.0 International license.

Figure S2

A

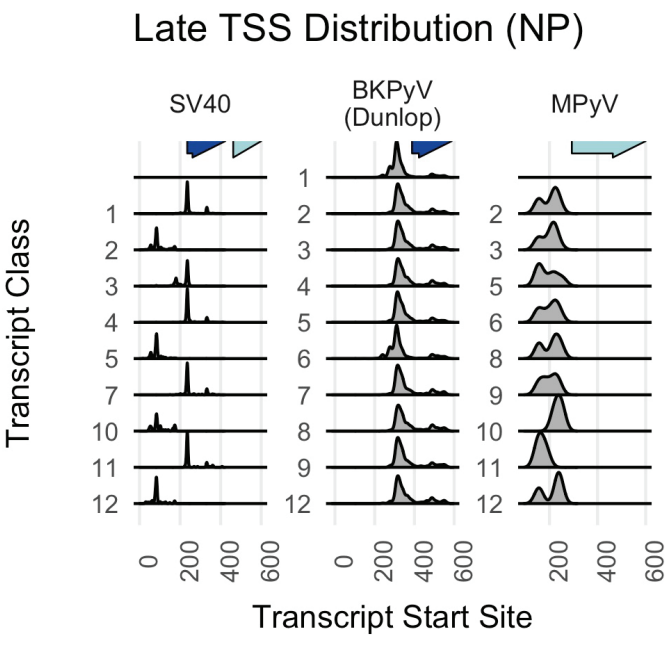

B Early TSS Distribution (NP)

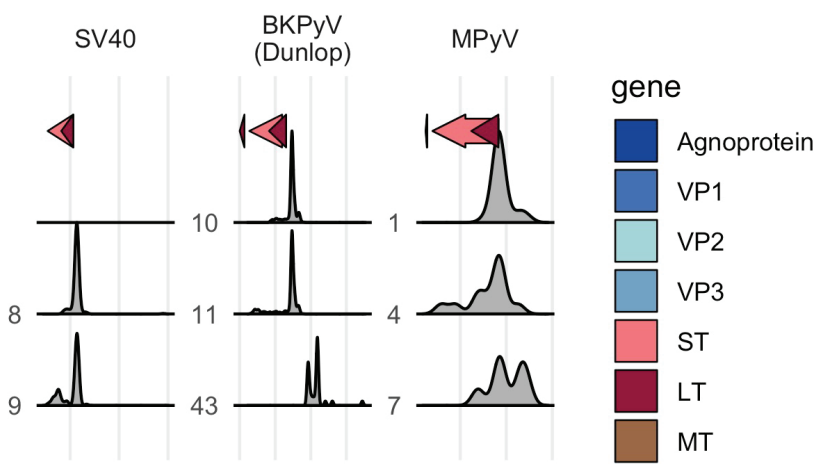

C

PolyA Tail Length Distributions

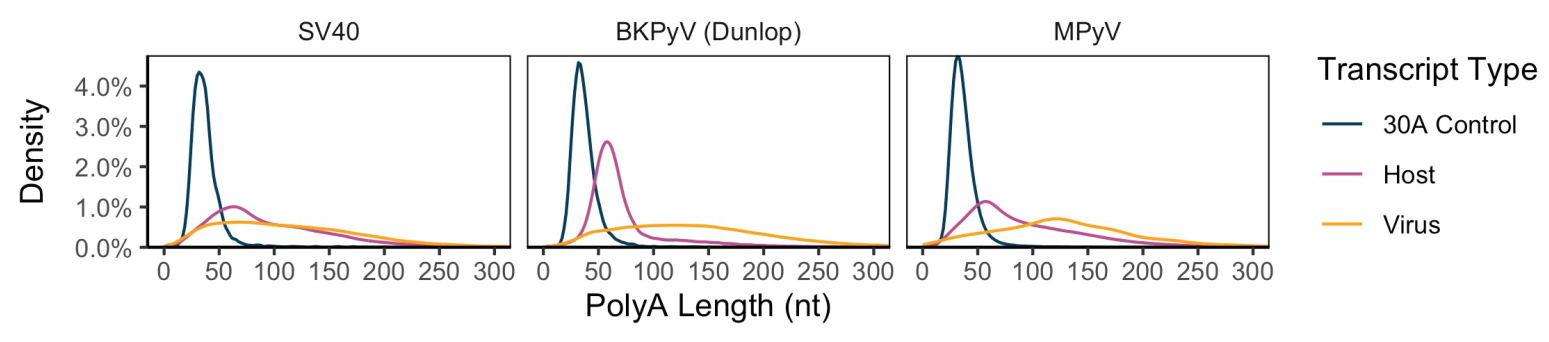

D

Ribosome occupancy of host transcripts in SV40-infected cells

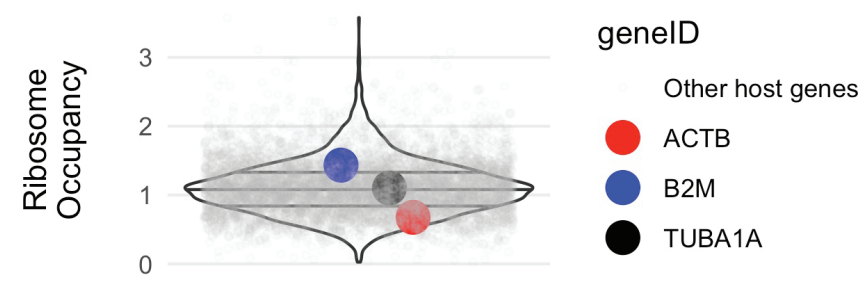


bioRxiv preprint doi: https://doi.org/10.1101/2021.11.12.468421; this version posted November 13, 2021. The copyright holder for this preprint (which was not certified by peer review) is the author/funder, who has granted bioRxiv a license to display the preprint in perpetuity. It is made FigS3: SV40 Transcriptome available under aCC-BY-NC-ND 4.0 International license.

A SV40: dRNAseq + SMRTseq, Late

${ }^{B}$ SV40: dRNAseq + SMRTseq, Early
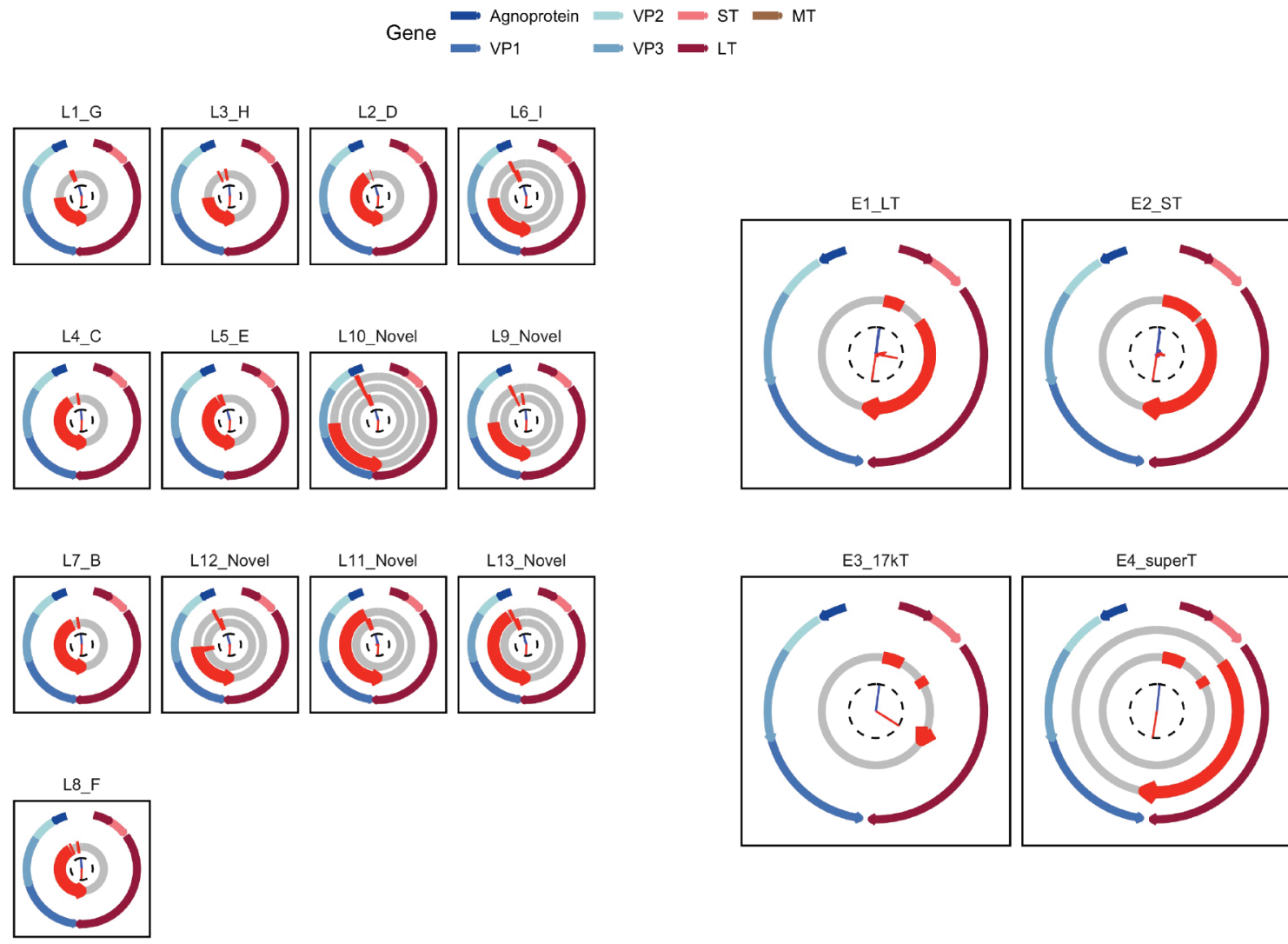

C

SV40: SMRTseq Only

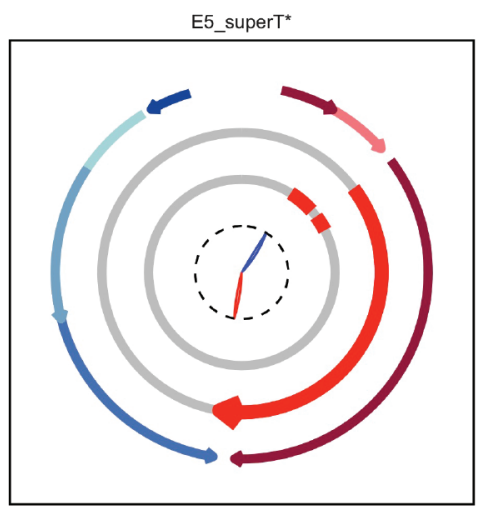

D

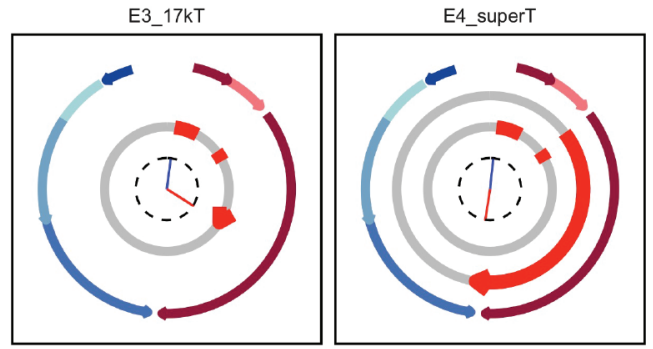

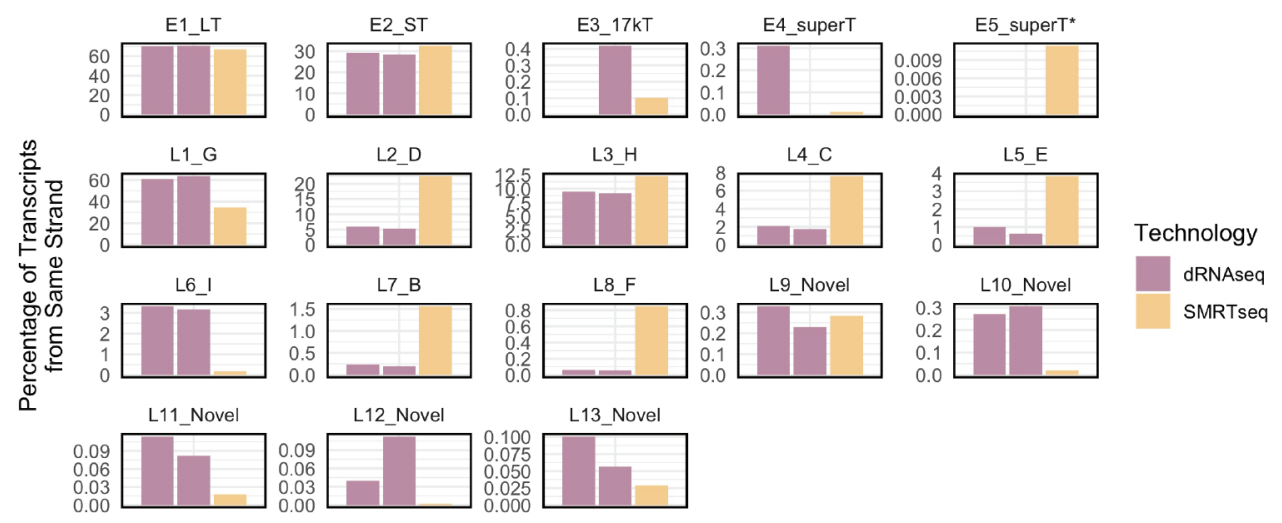


bioRxiv preprint doi: https://doi.org/10.1101/2021.11.12.468421; this version posted November 13, 2021. The copyright holder for this preprint (which was not certified by peer review) is the author/funder, who has granted bioRxiv a license to display the preprint in perpetuity. It is made FigS4: BKPyV (Dunlop) Transcriptome

A BKPyV (Dunlop): dRNAseq + SMRTseq, Late

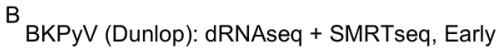

$$
\text { Gene }- \text { Agnoprotein }- \text { VP2 }- \text { ST }- \text { MT }
$$
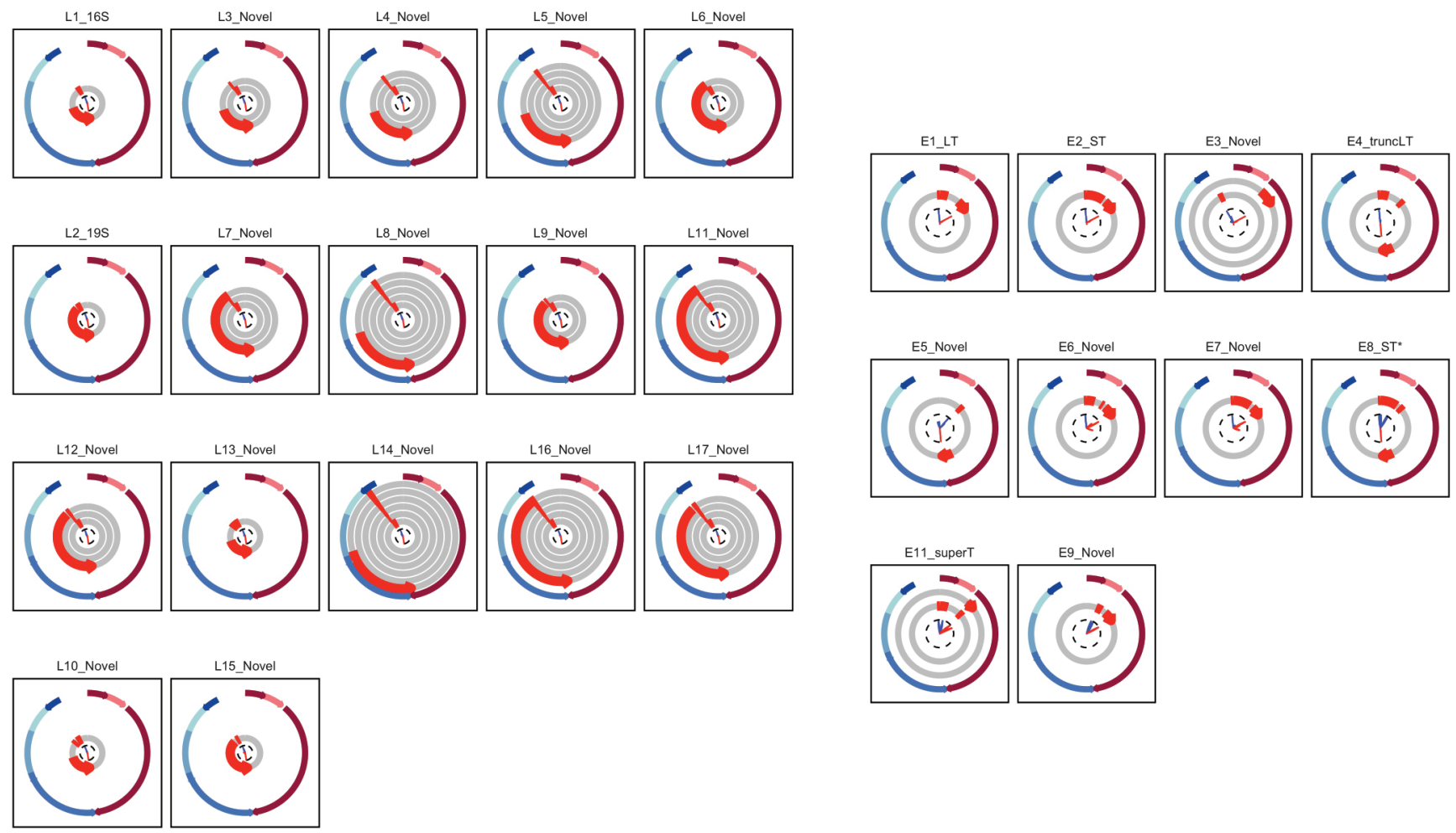

$\mathrm{C}_{\mathrm{B}}$

BKPyV (Dunlop): dRNAseq Only
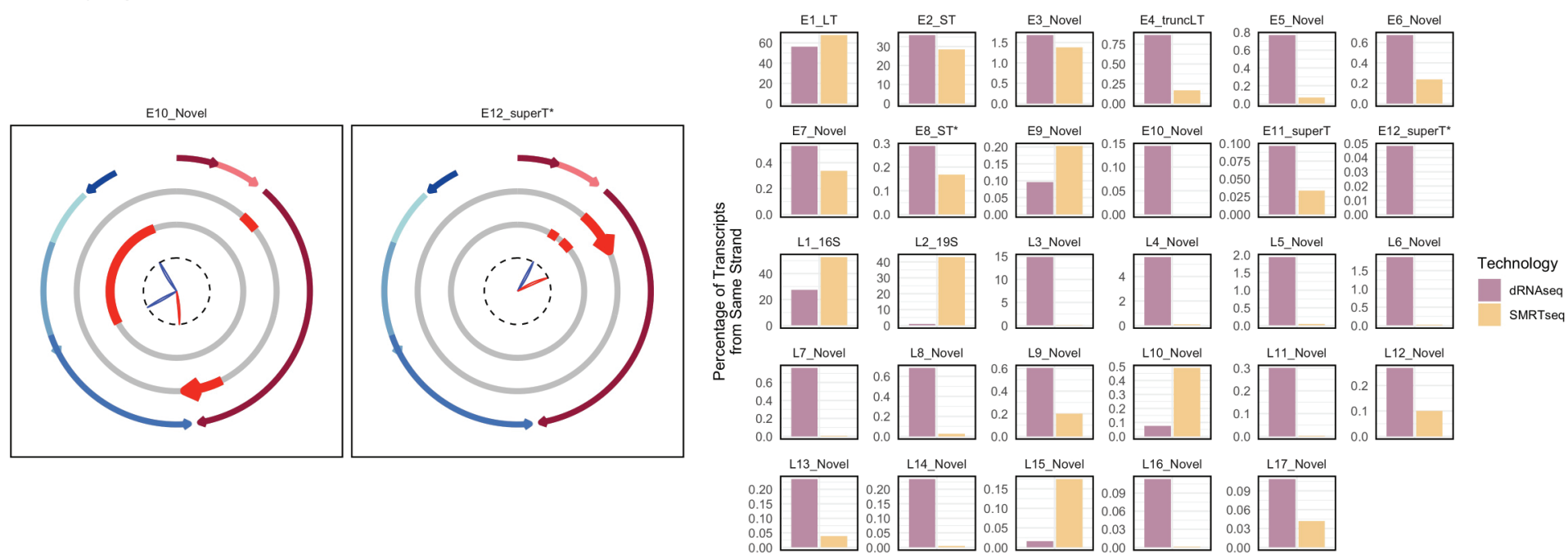
bioRxiv preprint doi: https://doi.org/10.1101/2021.11.12.468421; this version posted November 13, 2021. The copyright holder for this preprint (which was not certified by peer review) is the author/funder, who has granted bioRxiv a license to display the preprint in perpetuity. It is made available under aCC-BY-NC-ND 4.0 International license.

Fig S5: SV40 Late Transcript Pileups

A

SV40 Late: dRNAseq
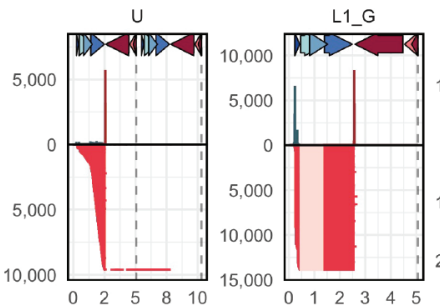

L6_I

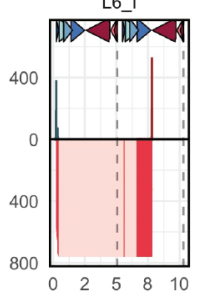

L10_Novel

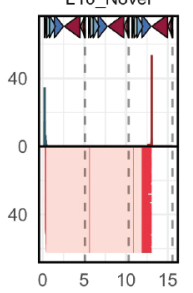

L8 F

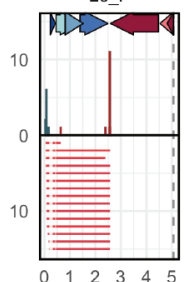

L4_C

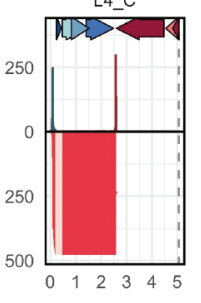

L7 B

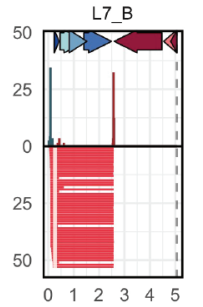

L12 Novel
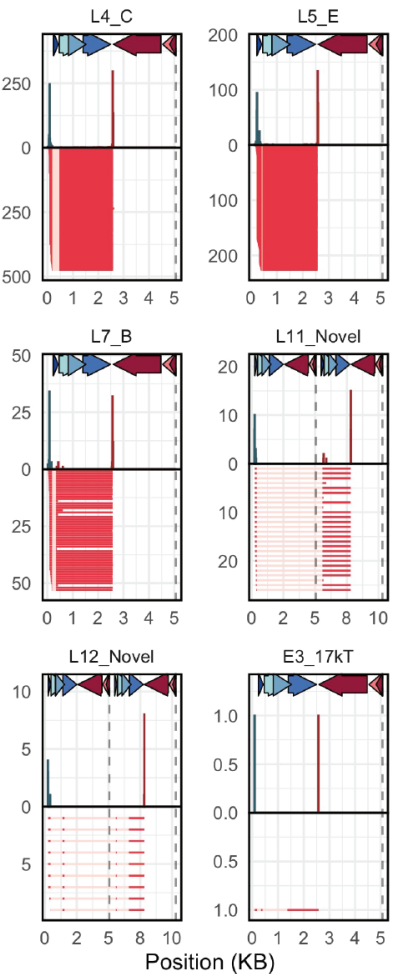

L11_Novel

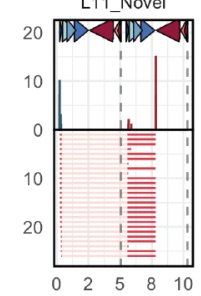

B

SV40 Late: SMRTseq
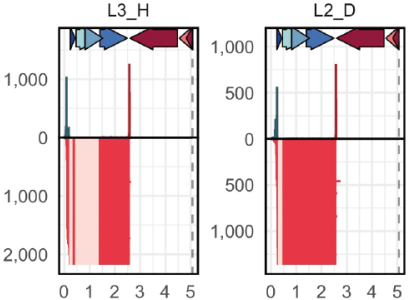

L9_Novel

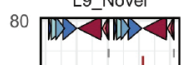

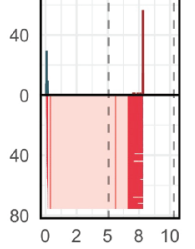

L13_Novel
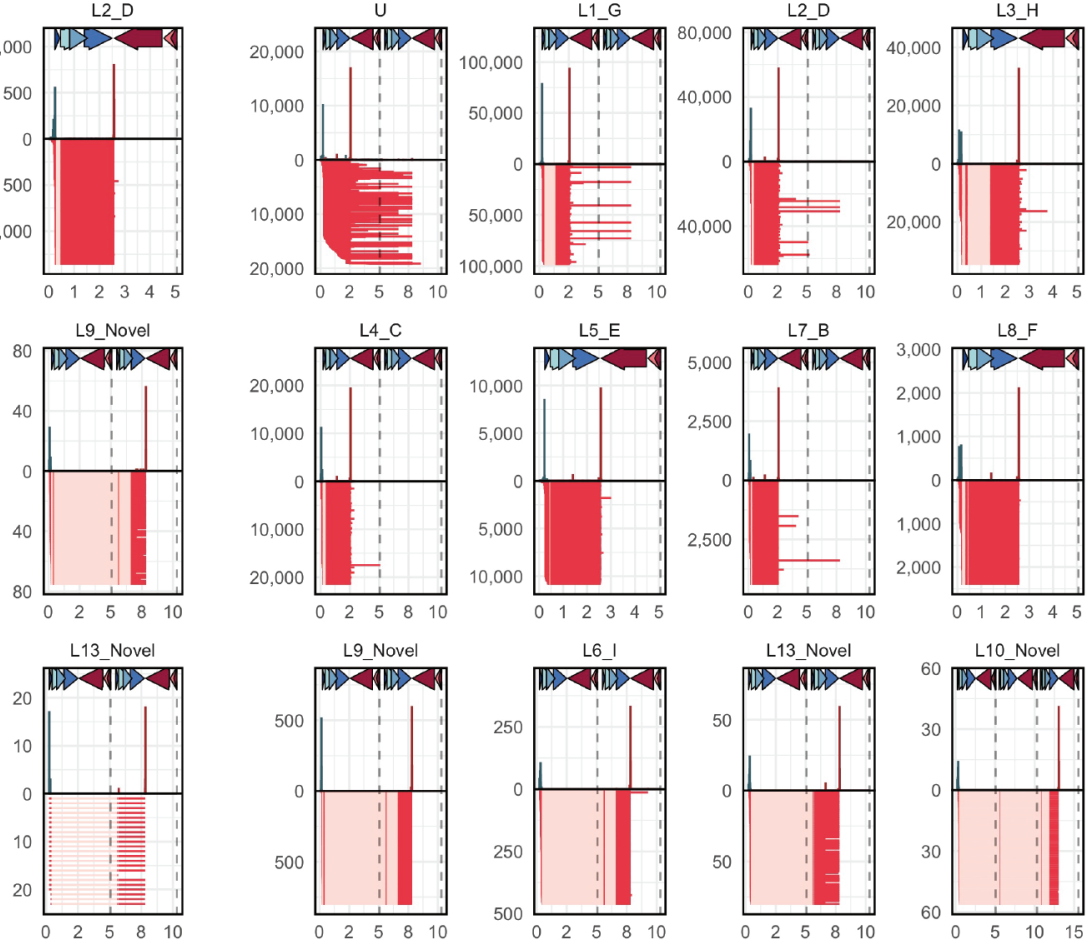

L4_C
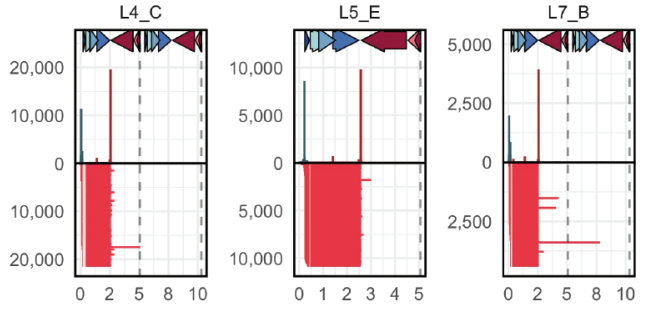

L6 I
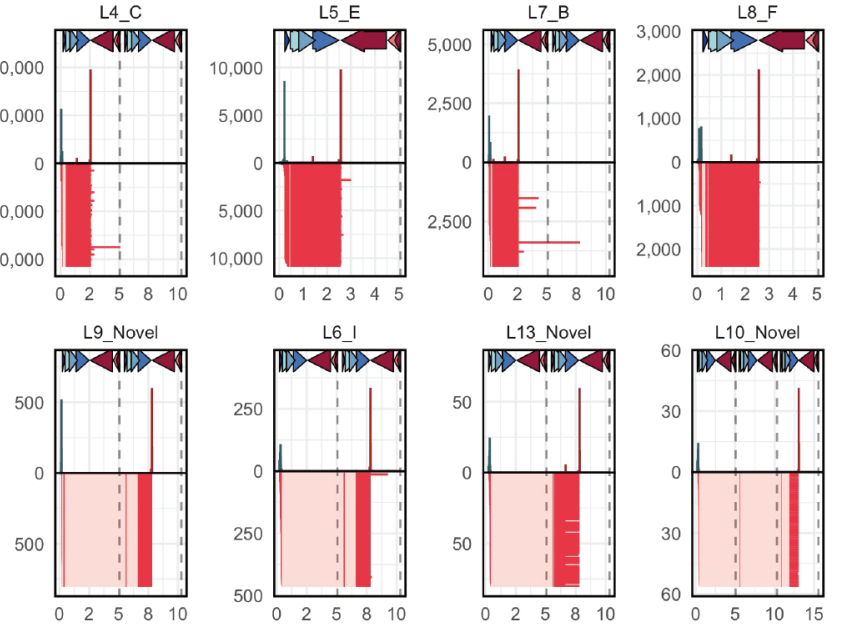

span_type

- exon

intron

gene

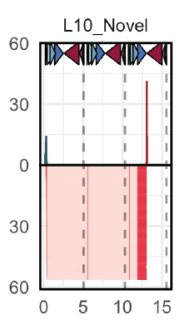

L11 Novel

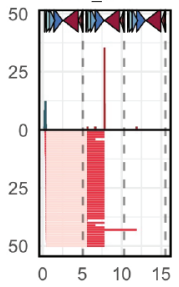


bioRxiv preprint doi: https://doi.org/10.1101/2021.11.12.468421; this version posted November 13, 2021. The copyright holder for this preprint (which was not certified by peer review) is the author/funder, who has granted bioRxiv a license to display the preprint in perpetuity. It is made available under aCC-BY-NC-ND 4.0 International license.

Fig S6: SV40 Early Transcript Pileups

A

SV40 Early: dRNAseq

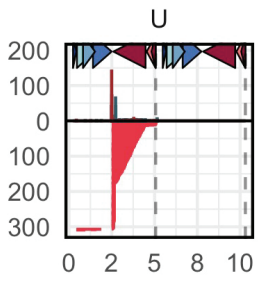

E3_17kT
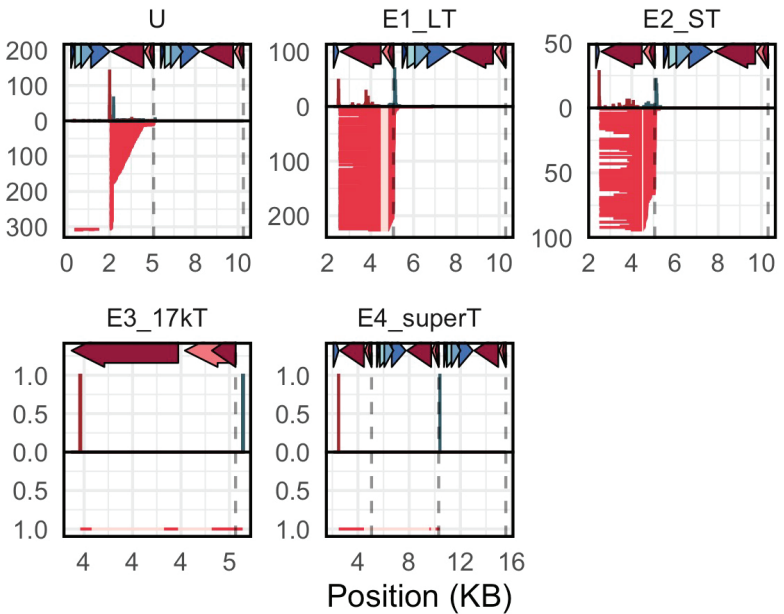

B

SV40 Early: SMRTseq

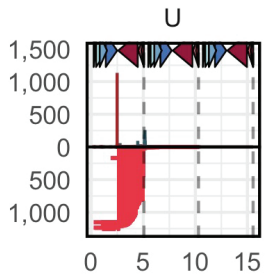

E3 $17 \mathrm{kT}$

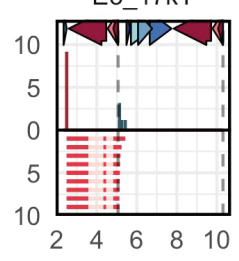

E1 LT

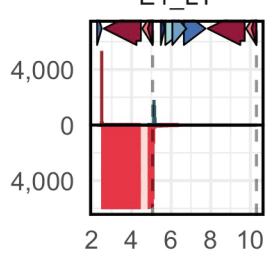

E4_superT

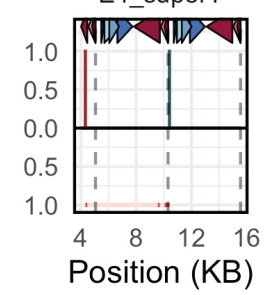

E2_ST

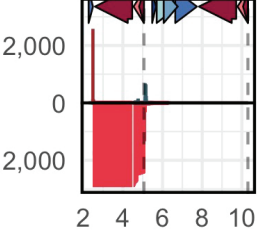

E5 superT*

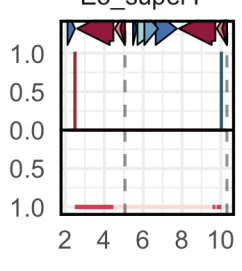

span_type

- exon

intron

gene

Agnoprotein

VP1

VP2

VP3

ST

$\mathrm{LT}$

MT 
bioRxiv preprint doi: $h t t p s: / / d o i . o r g / 10.1101 / 2021.11 .12468421$; this version posted November 13,2021 . The copyright holder for this preprint (which was not certified by peer review) is the author/funder, who has granted bioRxiv a license to display the preprint in perpetuity. It is made Fig S7: BKPyV (Dunlop) Late Transcript Pileups

A

BKPyV (Dunlop) Late: dRNAseq

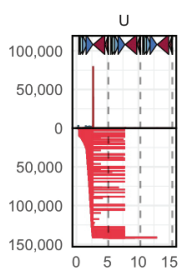

L1_16S

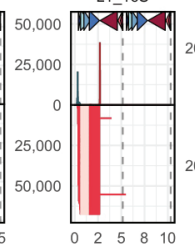

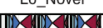
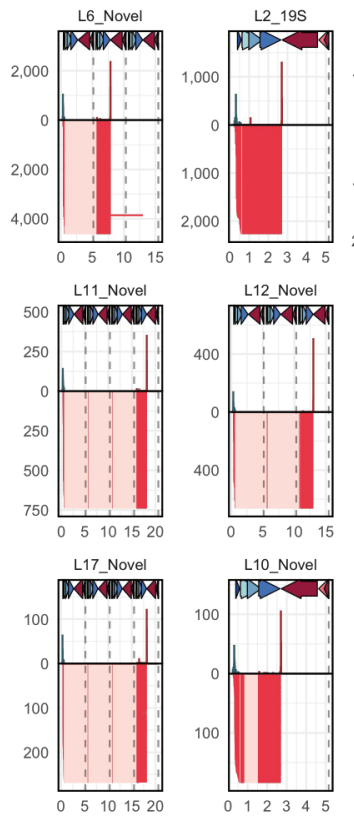

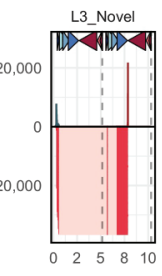

L7_Novel
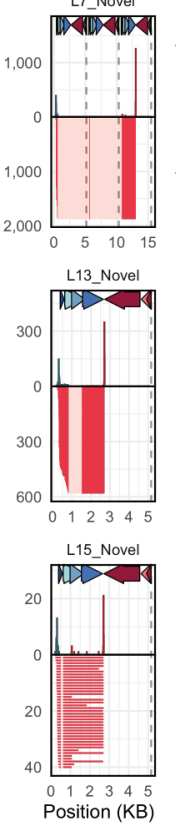
available under aCC-BY-NC-ND 4.0 International license.

B BKPyV (Dunlop) Late: SMRTseq
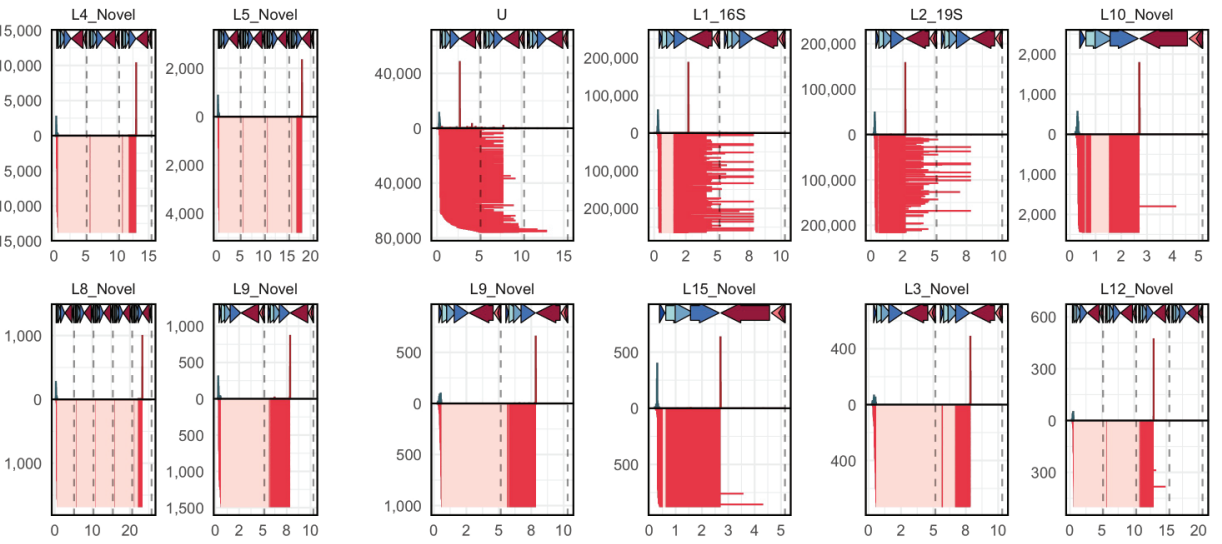

L9_Novel
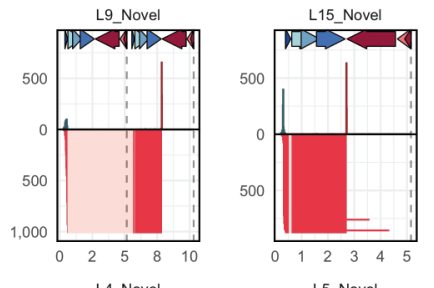

600 L14_Novel

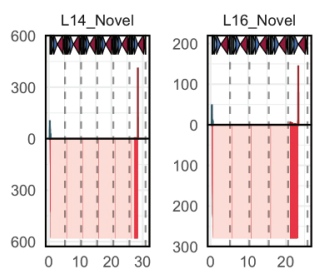

L4_Nove
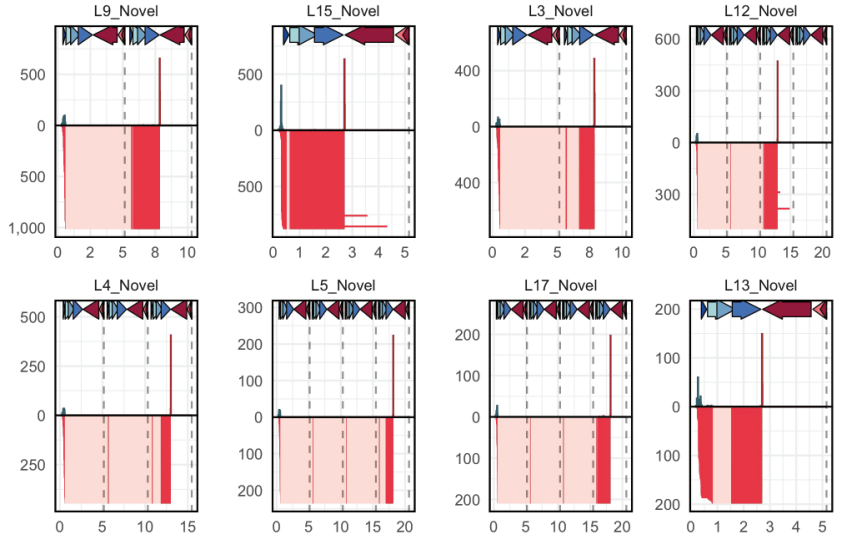

span type

- exon

intron
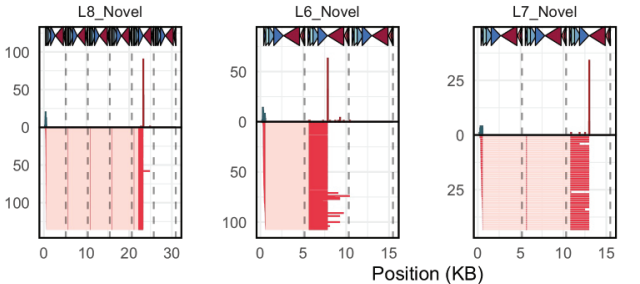
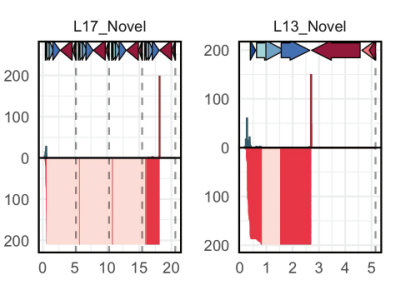

gene

Agnoprotein VP1 VP2

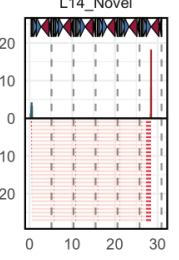


bioRxiv preprint doi: https://doi.org/10.1101/2021.11.12.468421; this version posted November 13,2021 . The copyright holder for this preprint (which was not certified by peer review) is the author/funder, who has granted bioRxiv a license to display the preprint in perpetuity. It is made Fig S8: BKPyV (Dunlop) Early Transcript Pileups

A

BKPyV (Dunlop) Early: dRNAseq
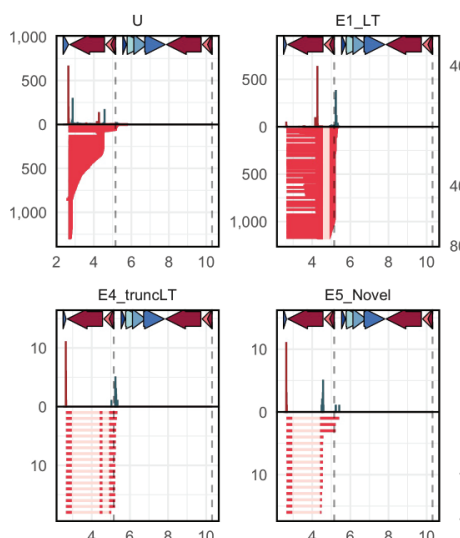

E8_ST

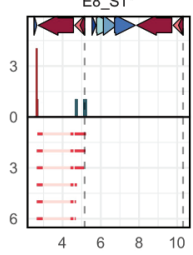

E12 super T*
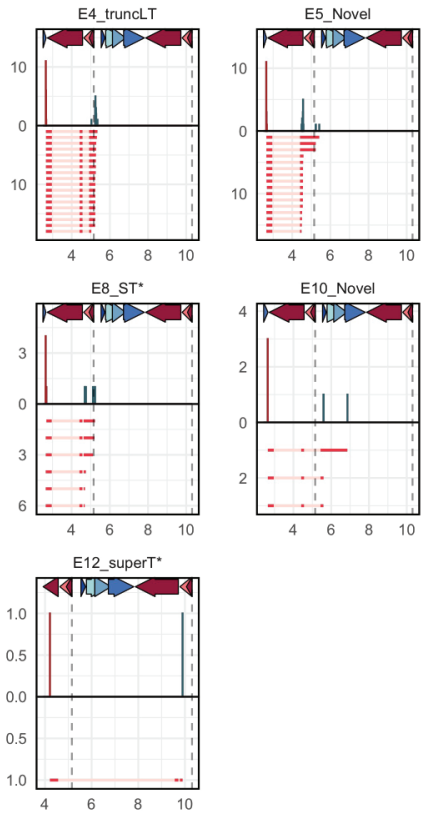
available under aCC-BY-NC-ND 4.0 International license.

B
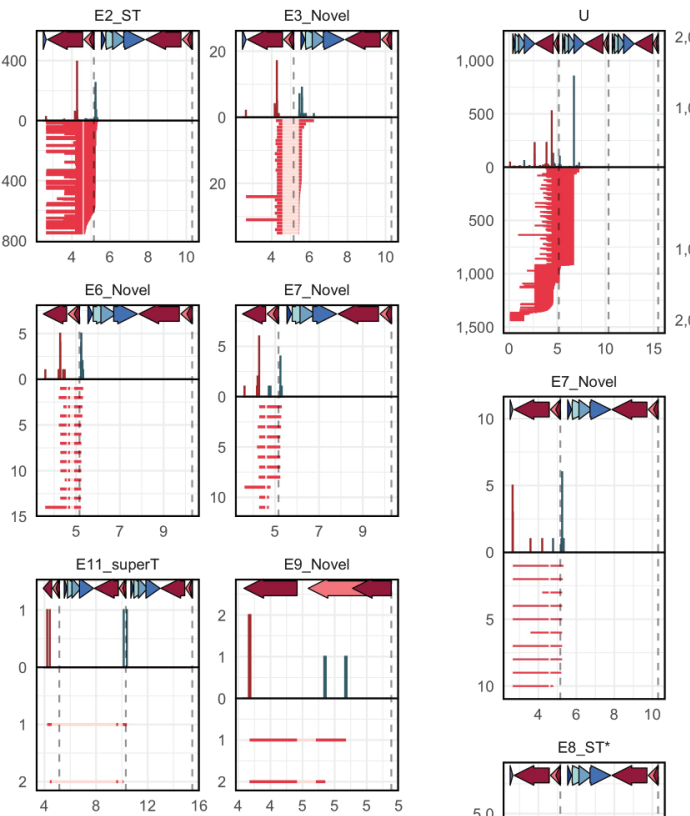

Position (KB)

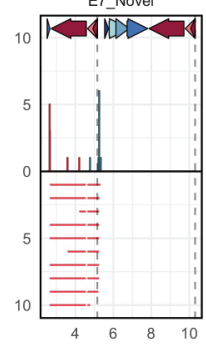

E8 $\mathrm{ST}^{*}$

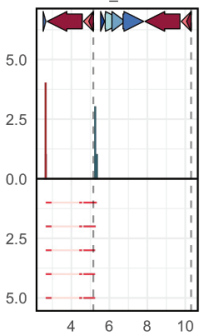

BKPyV (Dunlop) Early: SMRTseq

E1_LT

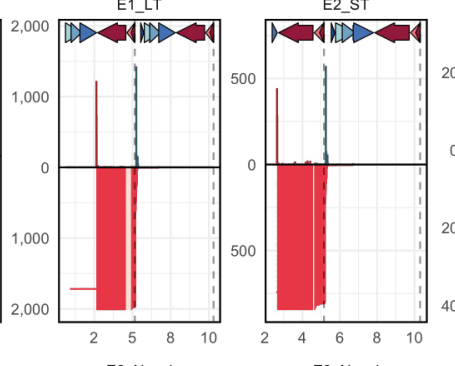

E9 Novel

E6 Novel

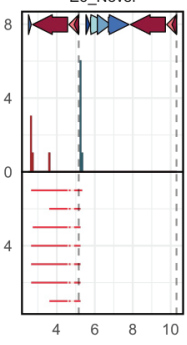

$8 \longdiv { \angle Y N D C - 1 }$

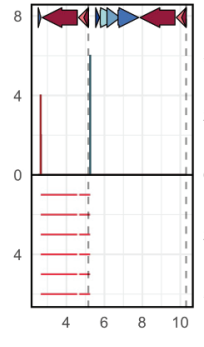

KAIDD $<1$

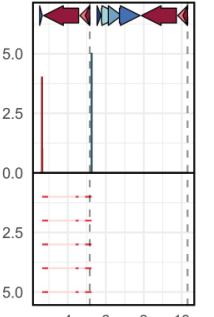

gene

Agnoprotein

VP1

VP2

VP3

LT

MT

pan_type

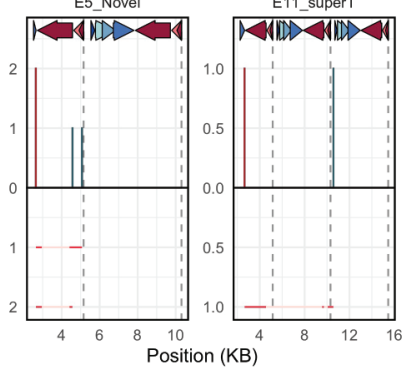

- exon 

Fig S9 (which was not certified by peer review) is the author/funder, who has granted bioRxiv a license to display the preprint in perpetuity. It is made Illumina Introns $>1 \%$ of Junction-spanning reads (by strand) available under aCC-BY-NC-ND 4.0 International license.

A
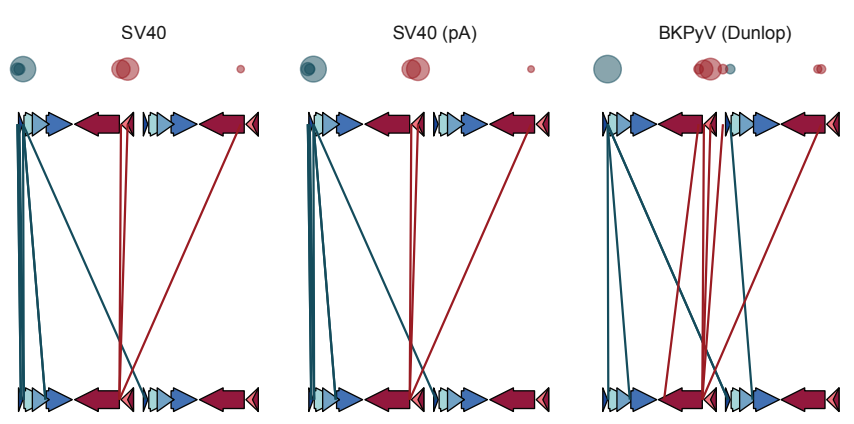

$\infty$ ○.

$\operatorname{BKPyV}($ Dunlop) (pA)
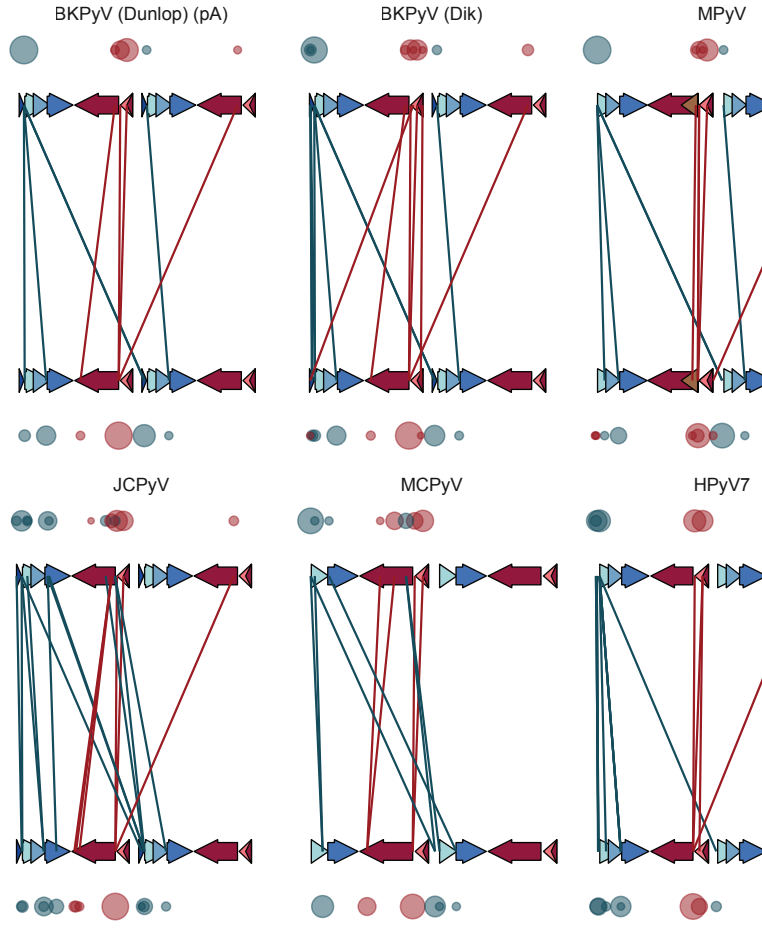

BSPyV1

(2)
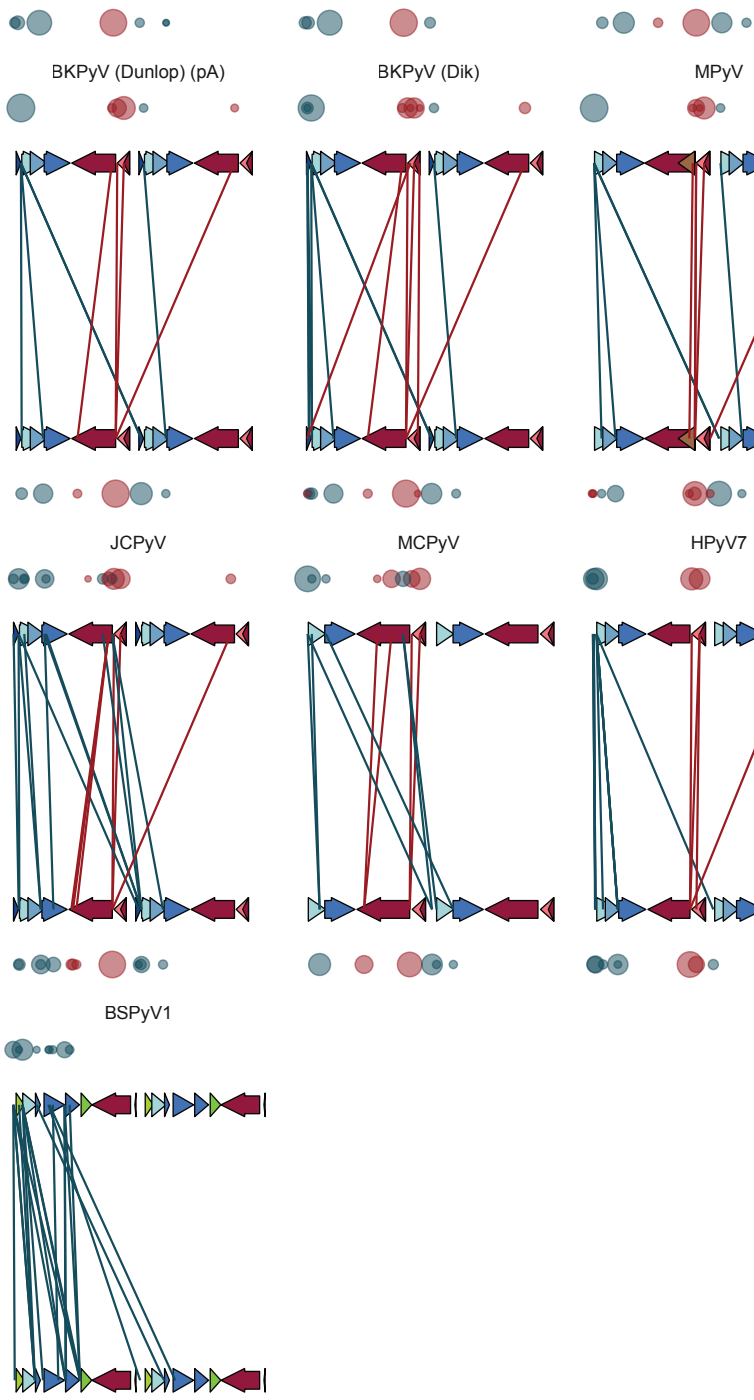

HPyV7
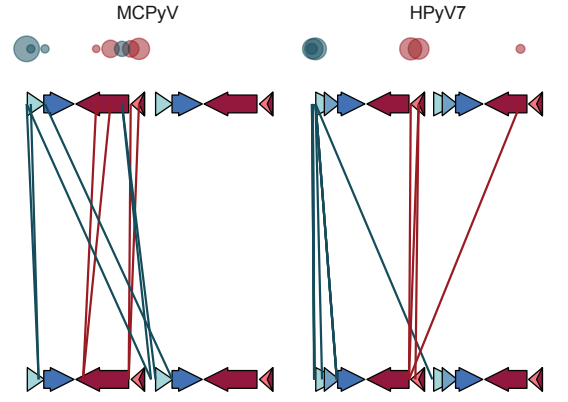

00
B
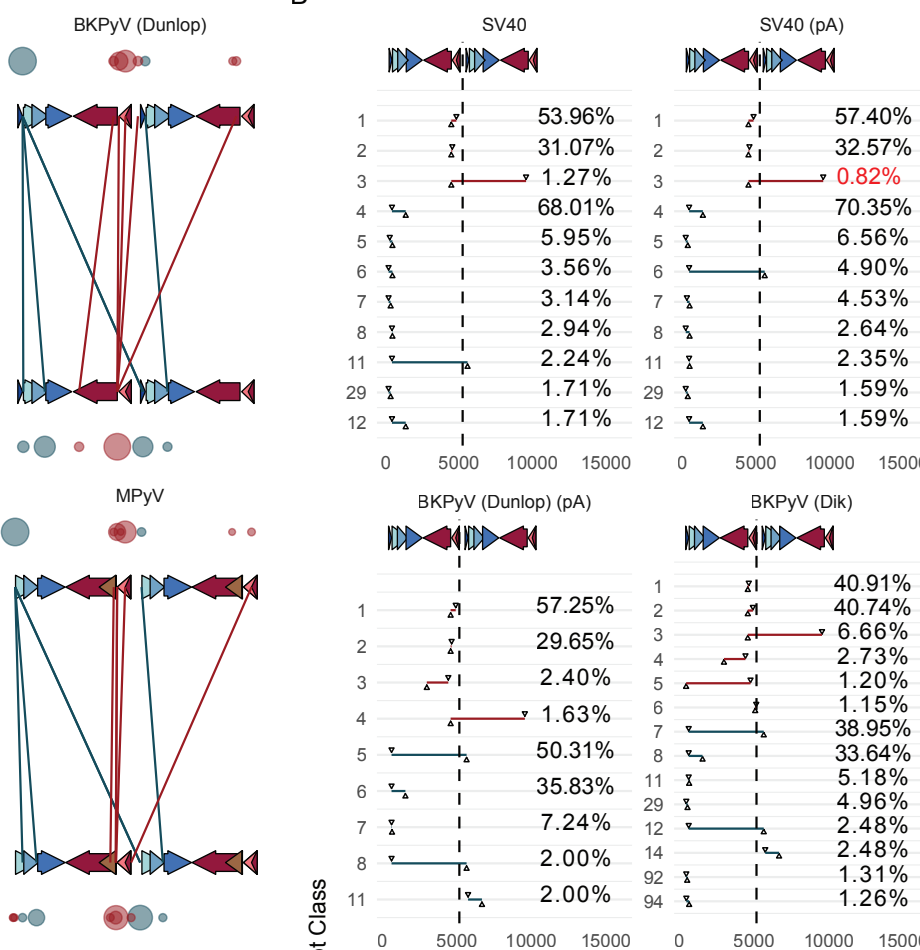

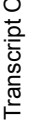

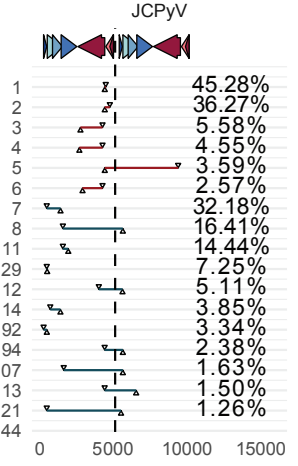

BSPyV1

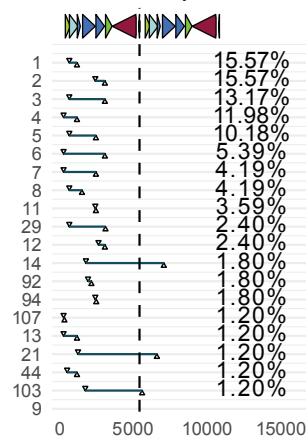

BKPyV (Dik)

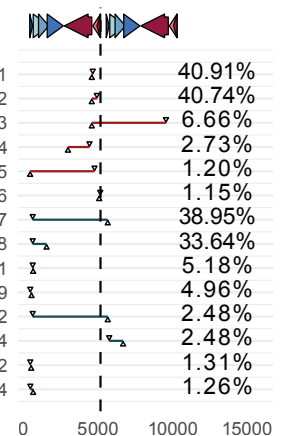

BKPyV (Dunlop)

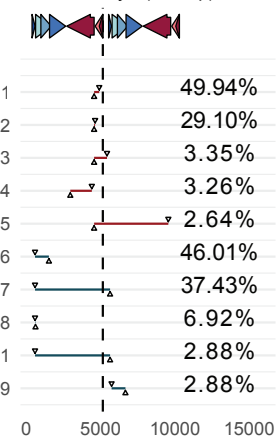

MPyV

\section{DD $\triangle M I D D<M$}

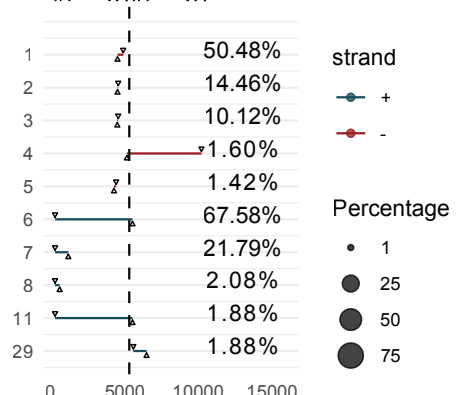

$0 \quad 5000 \quad 10000 \quad 15000$
0
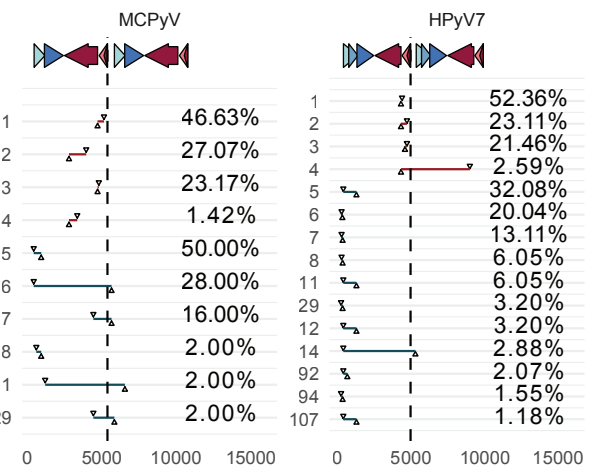

gene

Agnoprotein

VP1 VP2 VP3 ST ST LT MT Cygno VP1e 
bioRxiv preprint doi: https://doi.org/10.1101/2021.11.12.468421; this version posted November 13, 2021. The copyright holder for this preprint (which was not certified by peer review) is the author/funder, who has granted bioRxiv a license to display the preprint in perpetuity. It is made Figure $\mathbf{S 1 0}$

A

LT and ST Transcripts Watch Plots
D

Alternative Polyadenylation of
Early Spliced Transcripts
E

LT and ST
Transcripts

PolyA Lengths

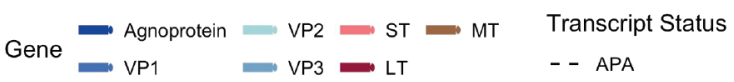

$$
\begin{aligned}
& \text { - Canonical }
\end{aligned}
$$

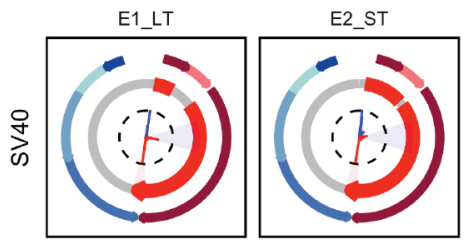

B

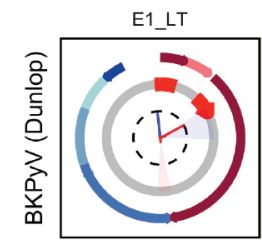

C

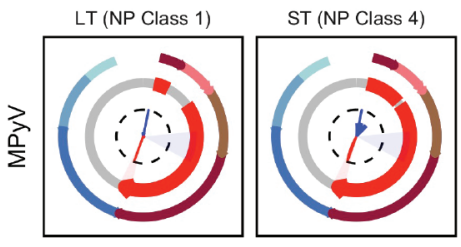

E2_ST

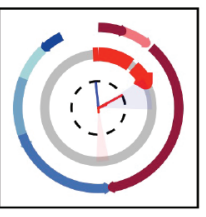

ST (NP Class 4) 음

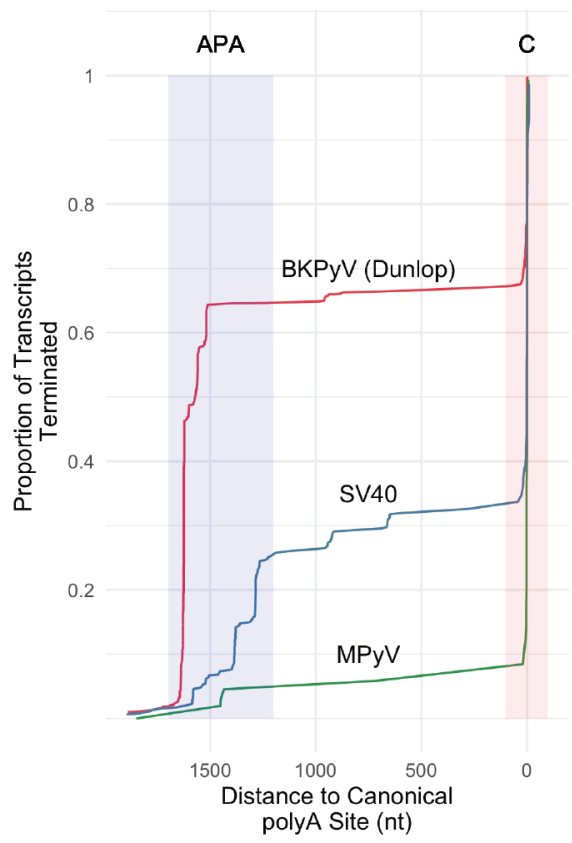

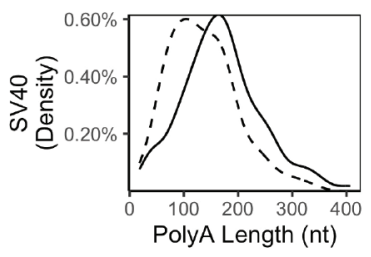

F

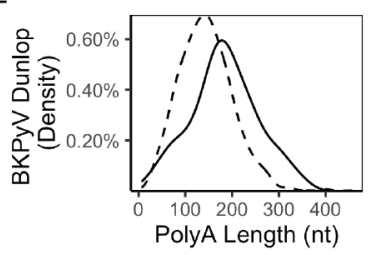

G

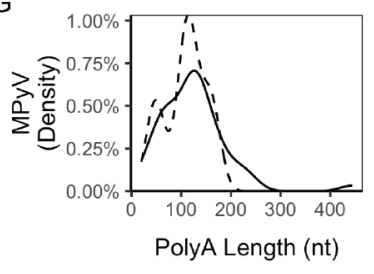


bioRxiv preprint doi: https://doi.org/10.1101/2021.11.12.468421. this version posted November 13,2021 . The copyright holder for this preprint (which was not certified by peer review) is the author/funder, who has granted bioRxiv a license to display the preprint in perpetuity. It is made Fig S11 available under aCC-BY-NC-ND 4.0 International license.

BKPyV Dik short-RNAseq (polyA)

A

BKPyV Dik WT (pA)

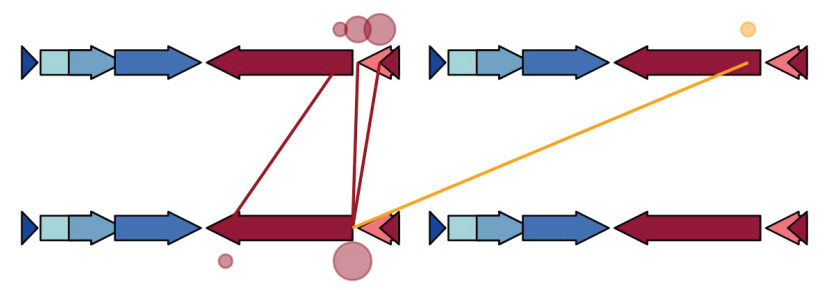

B

BKPyV Dik M1 (pA)

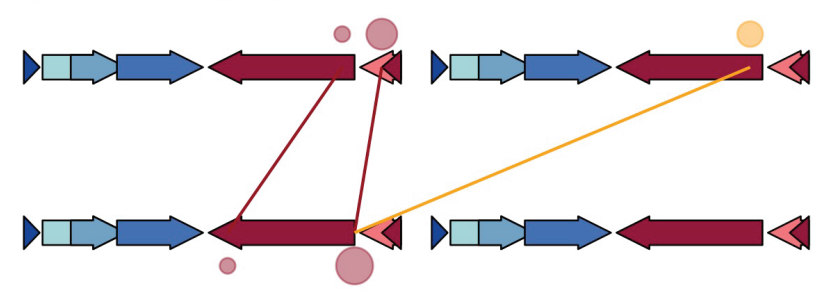

C

BKPyV Dik M2 (pA)

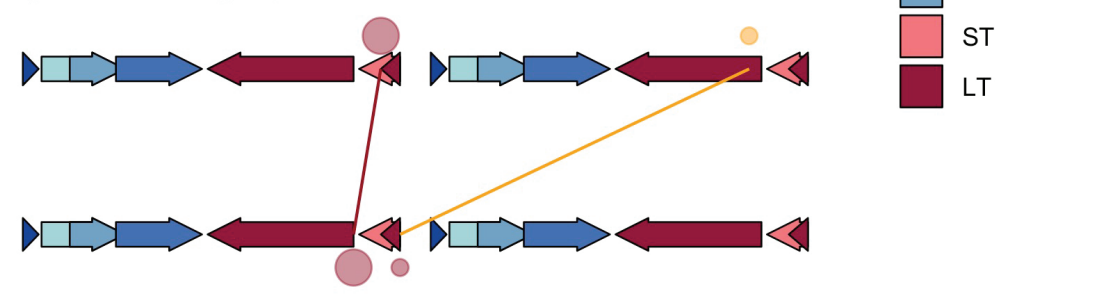

Percentage

- 1

- 25

○ 50

○ 75

gene

$\square$ Agnoprotein
$\square$ VP1

VP2

VP2

VP3

ST
LT 


\section{A Tumor: J45_440}

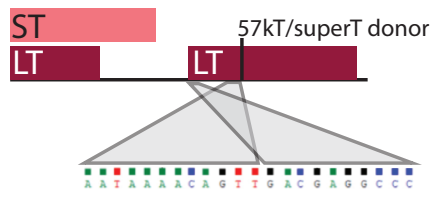

C Tumor: J45_440

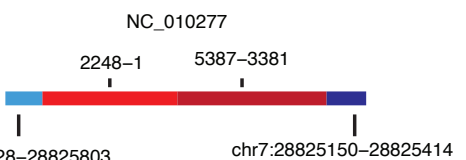

B

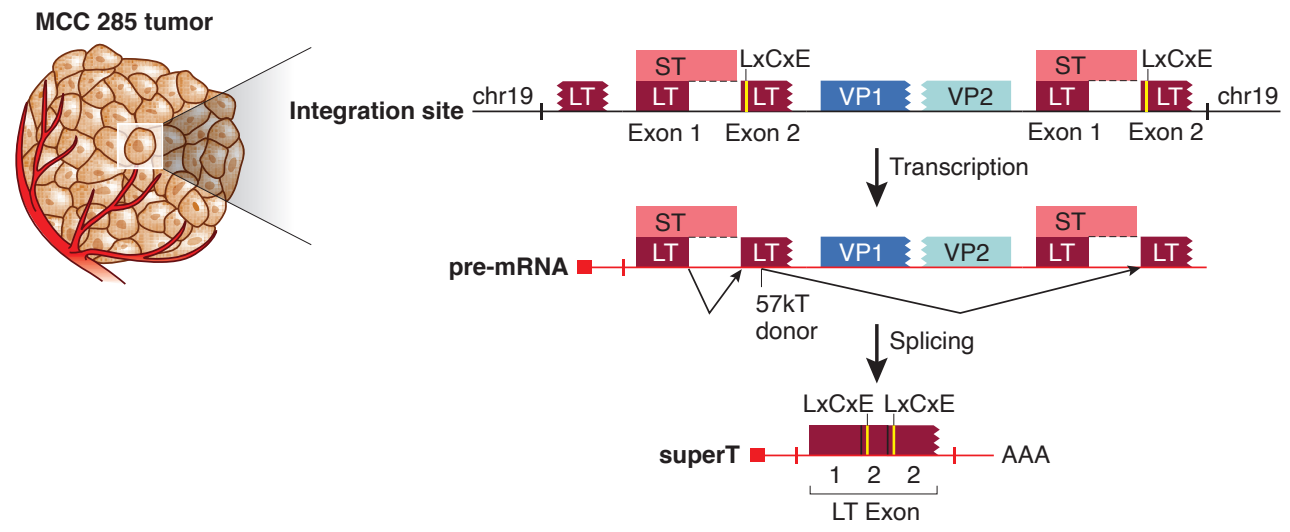

D Tumor: J17_296

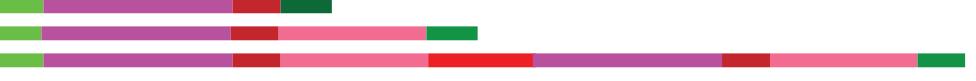

— chr2:174708385-174708543

- chr2:174844338-174844520

— chr2:174844524-174844754

— NC_010277.2_revComp_NCCR_TAg:1-429

- NC_010277.2_revComp_NCCR_TAg:1691-2994

— NC_010277.2_revComp_NCCR_TAg:2980-5387 — NC_010277.2_revComp_NCCR_TAg:415-1705

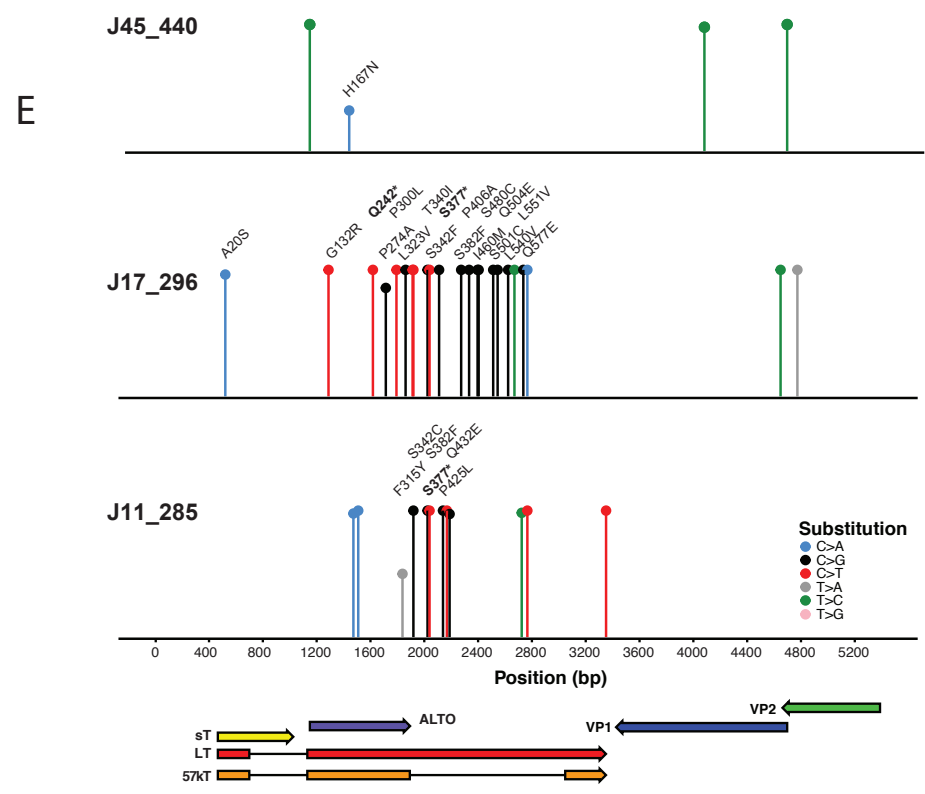

